\title{
Sleep and Wake Disorders Following Traumatic Brain Injury: A Systematic Review of the Literature
}

\author{
Catherine Wiseman-Hakes, ${ }^{\text {* Angela Colantonio, }},{ }^{2,3}$ and Judith Gargaro ${ }^{2}$ \\ ${ }^{1}$ Graduate Department of Rehabilitation Science, University of Toronto; ${ }^{2}$ Department of Occupational Therapy \\ and Occupational Science, University of Toronto; ${ }^{3}$ Toronto Rehabilitation Institute, Toronto, Ontario, Canada \\ Address all correspondence to Catherine Wiseman-Hakes, Graduate Department of Rehabilitation Science, Faculty of Medicine, University of \\ Toronto, 160-500 University Ave.Toronto, ON, M5G 1V7, Canada; Tel.: 416-946-8575; Fax. 416-946-8570; catherinewh@neurorehab.ca
}

\begin{abstract}
Traumatic brain injury is a leading cause of death and disability in both Canada and the United States. Disorders of sleep and wakefulness are among the most commonly reported sequelae postinjury, across all levels of severity. Despite this, sleep and wakefulness are neither routinely, nor systematically, assessed and are only recently beginning to receive more clinical and scientific attention. Objectives: This review aims to systematically appraise the literature regarding sleep and wake disorders associated with traumatic brain injury according to the following domains: epidemiology, pathophysiology, neuropsychological implications, and intervention; to summarize the best evidence with a goal of knowledge translation to clinical practice; and to provide recommendations as to how the field can best be advanced in the most scientifically rigorous manner. Methods: Systematic review and rating of the quality of evidence. Results: Forty-three articles were reviewed for levels and quality of evidence. Fifty-six percent of the literature was classified as Level III, and 24 percent were Level IVA. Overall, 89 percent of the studies were rated as moderate quality. A separate summary was provided for the pediatric literature. Conclusions: A comprehensive review of the emerging literature revealed wide ranges in estimates for incidence and prevalence of sleep disorders following TBI depending on characteristics of patients, measures used, and length of follow-up. Few treatments have been found to be effective, and as such more research is recommended. Our overview of the pediatric literature shows that this is an important issue for survivors of all ages. Overall, further work is needed to fully understand this complex disorder and to identify appropriate and timely interventions.
\end{abstract}

KEY WORDS: traumatic brain injury, mild traumatic brain injury, sport-related concussion, sleep, sleep disorders, insomnia, hypersomnia, excessive somnolescence, treatment

\section{BACKGROUND AND INTRODUCTION}

\section{A. Overview and Objectives}

Traumatic brain injury (TBI), defined as an acquired nondegenerative, noncongenital insult to the brain from an external mechanical force, is a leading cause of death and disability in both Canada and the United States for both adults and children. ${ }^{1}$ Furthermore, although the literature re- viewed in this paper is truly international in scope, at this time in the United States, "the nature of modern tactical engagement...in the current Iraq and Afghanistan conflicts has resulted in the largest proportion of identified traumatic brain injuries in any conflict in the nation's history." 2(p. 1004) In fact, the US Department of Defense reported an incidence of 10,963 diagnosed conflict-related TBIs in the year 2000. However, this incidence was reported as 27,862 by December 31, 2009, 
which represents an increase of $145 \% .^{3}$ TBI can result in significant impairments in physical, neurocognitive, and psychosocial/emotional function, as well as impairments in communication and sleep. Regardless of injury severity, these impairments may be transient or permanent, resulting in profound disruption for survivors and their families.

Disorders of sleep and wakefulness are among the most commonly reported sequelae postinjury, across all levels of severity. ${ }^{4,5}$ Despite this, sleep and wakefulness are neither routinely, nor systematically, assessed postinjury, and are only recently beginning to receive more clinical and scientific attention. Previous reviews by Ouellet and colleagues,${ }^{6}$ Orff and colleagues, ${ }^{7}$ and most recently by Zeitzer and colleagues ${ }^{8}$ indicate that the field is still in a developmental stage and a body of literature is beginning to emerge. These previous reviews focused primarily on the prevalence and nature of sleep disorders and, to a lesser extent, on implications and effective treatment strategies among adult TBI survivors.

As of yet, there has been no systematic appraisal of the literature, nor has there been a review that has included literature pertaining to a pediatric population. The literature on pediatrics is of particular relevance given the fact that approximately 500,000 children aged younger than 17 years are hospitalized each year with TBIs in the United States. ${ }^{9,10}$ This statistic does not include those children with minor TBIs who were either seen by their family physician/pediatrician or remained undiagnosed. Furthermore, TBI is the leading cause of disability in those under the age of 24 years, and the peak age of occurrence for TBI is $15-24$ years. ${ }^{11}$ Thus, the unique contributions of this paper are: 1) to systematically update and extend previous reviews on sleep and brain injury ${ }^{6-8}$ by conducting a thorough critical appraisal of the methodological quality of the literature that includes both children and adults and then, based on the identified strengths, weaknesses, and inconsistencies in the current literature; 2) to summarize the best evidence that currently exists with a goal of translating knowledge to clinical practice; and 3) to provide specific recommendations as to how the field can best be advanced in the most scientifically rigorous manner, given the unique complexities of this disorder.
In summary, the objectives of this review are to systematically appraise the literature regarding sleep and wake disorders associated with TBI according to the following domains: epidemiology (including sports-related concussion), pathophysiology, neuropsychological implications, and intervention. Furthermore, we will identify practice points and provide recommendations for a research agenda, based on the current body of literature.

In order to provide readers with a clearer understanding of the importance of sleep (and wakefulness) in the rehabilitation and recovery process, we begin with a brief definition of sleep and an overview of the role and functions of sleep.

\section{B. Definition of Sleep and Its Functions}

To fully grasp the significance of the impact of sleep disturbances postinjury, it is important to have a clear understanding of the actual definition of sleep and the critical roles and function it plays in human (and animal) physiology and homeostatic regulation. In 1995, Tobler ${ }^{12}$ defined sleep as a physiological, complex, and integrated behavior characterized by a significant reduction of the response to external stimuli, by a characteristic posture usually in a special environment, by a characteristic change in the neurophysiological recordings of brain activity, and by a homeostatic increase after its restriction. Sleep is a critical function that serves to reverse and/or restore biochemical and/or physiological processes that are progressively degraded during prior periods of wakefulness.

Sleep is believed to be essential for optimal immune function by influencing cellular (T-cell) immunity. Furthermore, an increase in growth hormone secretion is observed immediately following sleep onset, concurrently with a rise in cortisol in the latter half of sleep. This is critical for growth and development and is also important from the perspective of recovery from trauma, because not only does human growth hormone stimulate growth and cell reproduction, it also plays a critical role in cell regeneration. Furthermore, cortisol plays a crucial role in facilitating the body's adaptation to physical and mental stress, in the suppression of inflammation, enhancement 
of wound healing, modulation of plasma glucose, and increased production of erythrocytes (red blood cells). ${ }^{13}$

Sleep is divided into non-REM (rapid eye movement) and REM sleep. Studies of nonREM and REM sleep and the importance of their temporal succession in both animals and humans reveal that these stages have been found to play key roles in certain types of memory and learning, as well as cerebral plasticity. ${ }^{14-16}$ REM sleep, which occurs at the end of each sleep cycle, and the overall length of the REM cycle peaks prior to wakening also play a key role in aspects of memory consolidation and new learning. Furthermore, REM sleep during the neonatal period plays an important role in brain growth, neuronal maturation, connectivity, and synaptic plasticity in infants. ${ }^{17}$ Thus, recognition of the critical role that sleep plays in endocrine and immune function, thermoregulation, cognition, and behavior underscores the need to evaluate and optimize sleep during recovery from brain injury.

\section{METHODOLOGY}

An extensive and iterative literature search was conducted using the Ovid, Medline, PsychInfo, CINAHL, and EMBASE databases to ensure the capture of any relevant studies pertaining to the assessment of sleep after TBI. Medical subject heading terms included: brain injuries or brain concussion or brain hemorrhage, traumatic or brain injury, chronic or diffuse axonal injury/sleep disorders/insomnia, and hypersomnia. The search was limited to articles published in English from 1998-2009. Only peer-reviewed full-text articles were considered for this study.

The search was initially exhaustive to include titles relating to both acquired brain injury and TBI, as the terms are sometimes used synonymously. This allowed us to ensure that we captured all relevant studies. Each article abstract was then reviewed to further ensure relevance. Additional search strategies were also employed and as such, the reference lists of all articles were scanned for additional studies that may be relevant for review. Because the literature on the relationship between sleep disturbances and TBI is still developing, our inclusion criteria were quite broad and all identified articles were reviewed.

We then subdivided the articles into five different domains of scientific inquiry, including epidemiology, pathophysiology, pediatrics, intervention, and neuropsychological implications. Because there are a limited number of articles in the different subcategories of focus, all identified articles from Level II to Level V were included. In all, 68 articles were evaluated and 43 articles were included for the purposes of this review. We excluded only those that we deemed to be opinion papers or consensus papers not based on the results of a specific scientific study.

To critically appraise and stratify the quality of the evidence, the articles were evaluated on their research design and scientific rigor. Although it was not our intention to conduct a meta-analysis such as those undertaken by the Cochrane Collaboration Group, the ranking criteria were selected from the literature on evidence-based practice in rehabilitation, with a goal of providing guidance to end users about the studies likely to be most valid. Thus, the articles were categorized according to the scientifically validated hierarchy developed by Holm and edited by Boschen and colleagues (Table 1). ${ }^{18,19}$ Other rating systems are available; ${ }^{20-22}$ however, the Holm hierarchy ${ }^{18,19}$ was chosen in order to better differentiate among the published studies that exist at this point in the development of the literature on sleep and TBI. Given the early stage of development and the inherent complexities of the field, few randomized control trials (RCTs) have been conducted. It is, therefore, necessary to use a hierarchy that gives weight to non-RCT designs and also has a place for well-designed qualitative studies. Although the Holm hierarchy is an appropriate choice, it still did not fully differentiate the literature for our purposes. Thus, the edited and published version used by Boschen and colleagues ${ }^{19}$ was used for this systematic review.

Boschen and colleagues added two additional levels to the Holm hierarchy: the first is Level IIA, which was added to evaluate review papers that did not meet the criteria for Level I as they included non-RCT designs, and the second is Level IVA, which was added to separate out the well-designed non-experimental research designs such as cost analyses and the qualitative designs 
TABLE 1

Total Number of Articles Retrieved by Level of Evidence

\begin{tabular}{|c|c|c|}
\hline Level of Evidence & $\begin{array}{l}\text { No. of } \\
\text { Articles }\end{array}$ & Article Type \\
\hline Level la & 0 & Systematic reviews of randomized controlled trials (RCTs) \\
\hline Level II & 2 & Properly designed individual RCTs of appropriate size \\
\hline Level IIA ${ }^{b}$ & 2 & $\begin{array}{l}\text { Systematic reviews of case-controlled, cohort, or other experimental de- } \\
\text { signs (including RCTs reviewed together with other experimental designs) }\end{array}$ \\
\hline Level III & 25 & $\begin{array}{l}\text { Well-designed trials without randomization, single group pre-post, cohort, } \\
\text { time series, or matched case-controlled studies (also cross-sectional stud- } \\
\text { ies and case series) }\end{array}$ \\
\hline Level IV & 0 & $\begin{array}{l}\text { Well-designed non-experimental studies from more than one center or } \\
\text { research group }\end{array}$ \\
\hline Level IVA ${ }^{b}$ & 10 & $\begin{array}{l}\text { Well-designed individual non-experimental studies, cost analysis studies, } \\
\text { and case studies }\end{array}$ \\
\hline Level V & 6 & $\begin{array}{l}\text { Reports of expert committees, descriptive studies, clinical observations, } \\
\text { opinions of respected authorities, or testimonialsTotal }\end{array}$ \\
\hline Total Reviewed & 45 & \\
\hline
\end{tabular}

from the purely opinion and descriptive studies classified as Level V articles.

To fully capture the literature in its current state of evolution, we also considered review articles of multiple well-designed studies (called Level I and Level IIA studies in this hierarchy) in our article inclusion criteria. These review articles were considered separately from the single-study articles because they gave us a better perspective of the historical development of research in the field of sleep and TBI. Single studies were classified into levels based on the extent to which they met the criteria of the individual designated levels within this published hierarchy.

The process of assigning articles to levels was completed independently by two of the authors. Discrepancies were discussed and consensus was reached. Articles categorized as Level I or Level IIA were read and summarized but were not critically reviewed, as they were review articles in and of themselves. Published hierarchies do exist for the evaluation of systematic reviews; however, because none of the review articles identified for this systematic review were systematic in nature, these hierarchies were not applicable.
Each of the articles was evaluated using a template generated for use in this study. The templates addressed the purpose, study design, sample, outcome measures, significant findings, relevant conclusions, and comments made by the authors of the articles, as well as any special notes from the article reviewers. Two of the authors and an undergraduate student completed the templates for the articles.

In order to critically appraise the quality of the articles, information was abstracted from the templates in regard to the following seven questions:

1. Were baseline characteristics described and equivalence of groups evaluated?

2. Was the intervention/methodology described in detail, that is, sufficient for replication?

3. Was blinding employed?

4. What was the sample size per group used for data analysis?

5. What was the attrition rate?

6. Were the outcome measures used for the study standardized?

7. Was there a follow-up data collection point? 
This latter question was added specifically for the purposes of this review. The authors attempted to assess whether the intervention/ methodology was described in sufficient detail to allow for understanding of the key elements and to allow for replication. These items were identified based on the items used in meta-analytic and other systematic reviews. ${ }^{23}$ The presence of the above-listed seven quality elements and their description in the articles was further rated on a three-point scale developed by Boschen and colleagues $^{19}$ (Table 2). The classification of the quality rating - the sum of the scores of each of the six elements - was made using a trichotomous scheme representing "low" (summed score of $<3$ ), "moderate" (summed score of $\geq 3$ and $<5$ ), and "high" (summed score $\geq 5$ ) quality determined by the developers, based on the previous work of Boschen and colleagues. The abstraction and rating were performed independently by two of the authors and discrepancies were discussed and consensus was reached.

In addition, the articles on intervention were assigned a rating using the Downs and Black Rating System..$^{20}$ As the Downs and Black system is widely known and accepted, we attempted to use this for the evaluation of all of the articles included. However, although it is an excellent tool for the assessment of the quality of evidence specific to intervention studies, we encountered a number of difficulties in applying it to the nonintervention studies reviewed in this article. Thus, the Downs and Black System was applied only to the intervention studies. The use of these two quality assessment methodologies for the intervention studies allowed for comparison and a more thorough description of the literature.

\section{RESULTS}

\section{A. Previous Reviews of Sleep Disorders and Insomnia Following TBI: A Historical Overview of the Literature to Date}

To date, three reviews of the literature pertaining to sleep and/or insomnia following TBI have been published (Ouellet and colleagues, ${ }^{6}$ Orff and colleagues, ${ }^{7}$ and Zeitzer and colleagues ${ }^{8}$ ). The thorough Ouellet review, ${ }^{6}$ which we consider to be Level IIA, was the first to be conducted on the topic of sleep disturbance following TBI. This review made a number of important contributions to our understanding of the early adult literature on this complex topic. The authors aimed to re-

TABLE 2

Criteria Used to Assess the Quality of the Studies

\begin{tabular}{|c|c|c|c|c|c|c|c|}
\hline $\begin{array}{l}\text { Rating of } \\
\text { Assessed } \\
\text { Characteristic }\end{array}$ & $\begin{array}{l}\text { Baseline } \\
\text { Characteristics: } \\
\text { Described, } \\
\text { "Equivalence" }\end{array}$ & Blinding & $\begin{array}{l}\text { Sample } \\
\text { Size (per } \\
\text { group) }\end{array}$ & Attrition & $\begin{array}{l}\text { Standardized } \\
\text { Outcomes }\end{array}$ & $\begin{array}{l}\text { Description } \\
\text { of } \\
\text { Intervention }\end{array}$ & $\begin{array}{l}\text { Length } \\
\text { of } \\
\text { Follow- } \\
\text { Up }\end{array}$ \\
\hline 1 & $\begin{array}{l}\text { Both elements } \\
\text { present in the } \\
\text { article }\end{array}$ & Double & $>75$ & $<15 \%$ & All & $\begin{array}{l}\text { Sufficient to } \\
\text { replicate }\end{array}$ & $\begin{array}{c}>3 \\
\text { months }\end{array}$ \\
\hline 0.5 & $\begin{array}{l}\text { Either charac- } \\
\text { teristics were } \\
\text { described or } \\
\text { equivalence was } \\
\text { noted, but not } \\
\text { both }\end{array}$ & Single & $30-75$ & $15-25 \%$ & Some & $\begin{array}{l}\text { Some de- } \\
\text { scription }\end{array}$ & $\begin{array}{c}0-3 \\
\text { months }\end{array}$ \\
\hline 0 & $\begin{array}{l}\text { Neither } \\
\text { presented }\end{array}$ & $\begin{array}{l}\text { No blind- } \\
\text { ing or not } \\
\text { stated }\end{array}$ & $<30$ & $>25 \%$ & None & $\begin{array}{l}\text { No sufficient } \\
\text { description }\end{array}$ & None \\
\hline
\end{tabular}


view the evidence on epidemiology, etiology, and treatment of insomnia in the context of TBI, and to propose areas for future research.

With regard to the early literature on prevalence, Ouellet and colleagues ${ }^{6}$ reviewed nine studies and concluded that there were significant methodological issues in many of them, including the lack of an operational definition of insomnia, and heterogenous approaches (lack of consistency) to the assessment measures used to evaluate the presence and clinical significance of the insomnia. Furthermore, they identified that most of the studies varied in terms of the time of evaluation postinjury and the level of severity of the brain injury, making it difficult to draw any specific conclusions regarding prevalence.

Among the studies reviewed by Ouellet and colleagues, they identified a range of reported symptoms of insomnia from $30-70 \%$ in patients with TBI, with symptoms of poor sleep initiation and maintenance being the most prevalent. ${ }^{6}$ They also noted a range of time postinjury that symptoms (of insomnia) were present from 6-12 weeks postinjury up to several years. This was an important conclusion/observation because Ouellet and colleagues identified that "insomnia appears to develop a chronic course in a significant proportion of TBI patients." 6 (p. 188)

In addition, Ouellet and colleagues also identified a number of potential etiological factors that may be associated with the development of insomnia following TBI, including predisposing, precipitating, and perpetuating factors. ${ }^{6}$ Finally, they reviewed the literature on potential psychological and behavioral consequences of insomnia, and summarized the few studies on intervention, including pharmacotherapy and psychotherapies. The authors concluded the article with some basic practical recommendations based on the literature to date. They felt comfortable concluding that insomnia is a common problem after TBI, the etiology and maintenance of which is complicated. They further concluded that the presence of insomnia in patients with TBI likely has detrimental consequences; however, more research is needed to identify effective treatments. They suggested that because insomnia can be chronic, combinations of pharmacological and psychological treatment options should possibly be used. Data collected from non-TBI patients suggest that cognitive behavioral therapy (CBT) has promise.

In 2009, Orff and colleagues ${ }^{7}$ conducted an in-depth review of the literature, which we also identified as being Level IIA. This review, which builds on the previous work by Ouellet and colleagues, ${ }^{6}$ included a clearly defined search strategy, key search terms, and methodology. This review aimed to address "the etiology and implications of sleep problems in TBI, particularly mild TBI (mTBI), across 4 general domains of current scientific inquiry and observation: subjective impressions of poor sleep; objective changes in sleep-related parameters; alterations in circadian rhythms; and neurophysiologic and/ or neuropsychologic abnormalities associated with TBI." ${ }^{\text {(p. } 155)}$

In the five years since the Ouellet review, the prevalence of sleep disturbances following TBI (both subjectively reported and objectively measured, although the latter has some contrary findings) has been further established, and the literature has thus evolved to more closely look at this complex disorder. With regard to subjective impressions of poor sleep, Orff and colleagues? identified that the majority of studies surveyed had reasonably large sample sizes and provided replicable findings of insomnia in TBI patients, particularly those with mild TBI (mTBI). Results of the studies surveyed on the topic of objective changes in sleep parameters were not quite so conclusive. The authors identified several studies with objective evidence of changes in sleep quantity and quality, as well as other sleep disorders such as obstructive sleep apnea (OSA), periodic limb movements (PLM), and narcolepsy. However, they also cited a number of studies in which objective changes in sleep parameters were not found, despite subjective complaints of the subjects. The authors cited similar methodological concerns as those previously addressed by Ouellet and colleagues, and further cited the lack of, or inconsistent reporting on, other important factors such as prior TBIs and past/current medical and psychiatric status, "making it difficult to evaluate the role of these variables in sleep-related outcomes and complicating comparisons between studies." 7 (p. 163)

Orff and colleagues ${ }^{7}$ reviewed the literature on TBI and circadian changes, and reported that 
although the literature is relatively sparse and somewhat conflicting at this time, there is a growing body of evidence to suggest that sleep disturbances following TBI may be due to alteration in the timing and rhythms of sleep (i.e., circadian rhythm sleep disorders) in a subset of patients. In addition, they reviewed the literature on neurophysiologic and neuropsychological disturbances associated with sleep. They reported endocrine changes that may explain sleep disturbances in this population and also highlighted the limited but important research that suggests that deficits in cognitive performance after TBI may be related, in part, to the degree of sleep disturbance.

Consistent with the Ouellet review, Orff and colleagues also summarized the literature on interventions to date, which remains limited to a few studies addressing pharmacotherapy and CBT. Finally, they discussed limitations of the literature and identified that although the body of work has grown since the Ouellet review in $2004,{ }^{6}$ there remain similar methodological limitations such as small sample sizes, considerable variation in age, time since injury, level of severity, and measures used to assess sleep. Orff and colleagues recommend that future research needs to focus on uncovering the specific types, causes, and severity of TBI that most often lead to sleep problems; the consequences of sleep disturbance; and the most effective treatment strategies.

The final review studied for this article is by Zeitzer and colleagues. ${ }^{8}$ This review, which we identified as being a Level $\mathrm{V}$ and lacking the methodological strength of the previous two reviews, was conducted for the US Department of Veterans Affairs (VA). As such, its focus seems to consider insomnia in the context of TBI from the perspective of those assessing and treating veterans diagnosed with $\mathrm{mTBI}$, hampered by a co-morbid sleep disorder. (Note: Sleep apnea may in fact be preexisting because it is, interestingly, present in about half of the Department of VA patient population ${ }^{8[\mathrm{p} .}{ }^{829]}$ ). This review lacks a clearly defined objective and systematic review approach; however, the authors state that they will "consider insomnia directly caused by TBI (e.g., secondary to neural damage), indirectly caused by TBI (e.g., secondary to depression), and unrelated to TBI but occurring within individuals with TBI." 8 (p. 827)
Consistent with previous reviews, Zeitzer and colleagues cited studies of TBI populations of varying severities (mild-moderate) and varying lengths of time postinjury from 6 weeks to greater than 10 years. They reported that insomnia occurs in approximately $40 \%$ of individuals with a TBI of any severity, even though it is more commonly reported among patients with mTBI, and is the most prevalent somatic complaint across all levels of severity. The authors also postulated two components of TBI-related insomnia. First, there is a general predisposition to develop sleep disturbance as a result of alterations or disruptions in neurotransmitters involved in the regulation of sleep (and they state further that the literature on this topic is for the most part limited to those with moderate-severe TBI). Second, acute stressors may trigger insomnia. They highlighted non-sleep comorbidities such as depression, pain and post traumatic stress disorder, all of which are of particular relevance to individuals with TBI in general and to veterans with mTBI. From this discussion, they highlighted two future research questions. First, can mTBI cause disruptions in the regulation of sleep? The authors stated further that the literature on this topic is, for the most part, limited to those with moderate-severe TBI. Second, can acute stressors trigger the insomnia? They highlighted non-sleep comorbidities such as depression, pain, and posttraumatic stress disorder, all of which are of particular relevance to individuals with TBI in general and to veterans with mTBIs. They also identified two additional research questions: Can mTBI cause disruptions in the neurotransmitters critical for sleep and wakefulness?; and, What is the effect of psychiatric comorbidities on the occurrence or severity of insomnia in the context of TBI? The latter question aims to assist the physician in treating insomnia as a primary or secondary pathology.

Finally, Zeitzer and colleagues ${ }^{8}$ briefly summarized the literature on pharmacological and nonpharmacological treatments. As per the previous two reviews, the literature on treatment remains limited. As a result, these authors identified some cautionary caveats in the use of pharmacotherapy and described nonpharmacological interventions such as CBT plus various combinations of sleep hygiene, sleep restriction, and relaxation training. The authors highlighted the critical need for further 
nonpharmacological studies as viable alternatives to treatment of insomnia in patients with mTBI. The authors concluded that more systematic research is required to "provide a foundation for an evidence-based medical approach to treatment of insomnia in the context of mTBI." ${ }^{\text {(p. } 832)}$

\section{B. Single Study Papers}

As shown in Table 1, the majority of the literature (56\%) is classified as Level III (Well-designed trials without randomization, single group pre-post, cohort, time series, or matched case-controlled studies [also cross-sectional studies and case series]). The next largest level is Level IVA, with $24 \%$ of the categorized articles (well-designed individual non-experimental studies, cost analysis studies, and case studies). The published literature is getting stronger as there are fewer Level $\mathrm{V}$ studies than Level III or IVA studies, but is still clearly in development as there are no Level I studies and few Level II or IIA studies.

Single study papers were organized into the following categories: epidemiology (comparison group studies or single group/cohort studies), pathophysiology, pediatrics, neuropsychology, and intervention (see Tables 3, 4, and 5). It is important to note that there are Level III studies in each of the above categories, but as of yet the Level II studies are only with regard to pharmacological interventions. Only one of the studies reviewed was considered of low quality, the majority were of moderate quality (89\%), and only a few were of high quality $(10 \%)$. Each of the studies is summarized in detail in Table 5, which lists the level, quality, and key details relating to the conduction of the reported study. Each category of studies will be discussed below in the order in which they appear in the table. Following each summary there will be an in-text box summarizing the research agenda and practice points identified with the authors of the reviewed studies, followed by our recommendations.

\section{Epidemiology}

The evidence relating to the epidemiology of sleep and wake disturbances following TBI has historically been the primary focus of the literature, comprising 23 of the 43 articles across the five subtopics we identified for this review. Epidemiological investigation of a phenomenon is a necessary starting point as it provides a solid foundation for all further investigations to follow. We subdivided the studies on epidemiology, all of which were rated as Level III study designs, to include nine papers with a comparison group, all of moderate quality, and 12 articles that used a

\section{TABLE 3}

Article Scope and Level of Articles

\begin{tabular}{|c|c|c|c|c|c|}
\hline Article Scope & Total No. of Articles & Level II/IIA & Level III & Level IVA & Level V \\
\hline $\begin{array}{l}\text { Epidemiology - Comparison } \\
\text { group }\end{array}$ & 9 & & 9 & & \\
\hline $\begin{array}{l}\text { Epidemiology - Single group/ } \\
\text { cohort }\end{array}$ & 14 & & 5 & 6 & 3 \\
\hline Pathophysiology & 3 & & 2 & 1 & \\
\hline Pediatrics & 6 & & 6 & & \\
\hline Neuropsychology & 4 & & 1 & 2 & 1 \\
\hline Intervention & 6 & 2 & 3 & 1 & \\
\hline Review & 3 & 2 & & & 1 \\
\hline Total & 45 & $2 / 2$ & 26 & 10 & 5 \\
\hline
\end{tabular}


TABLE 4

\section{Article Scope and Quality of Articles}

\begin{tabular}{|c|c|c|c|c|c|c|c|}
\hline \multirow[t]{2}{*}{ Article Scope } & \multirow[t]{2}{*}{ Total } & \multicolumn{4}{|c|}{ Moderate Quality } & \multicolumn{2}{|c|}{ High Quality } \\
\hline & & 3 & 3.5 & 4 & 4.5 & 5 & 6.5 \\
\hline $\begin{array}{l}\text { Epidemiology - } \\
\text { Comparison group }\end{array}$ & 9 & & & 6 & 3 & & \\
\hline $\begin{array}{l}\text { Epidemiology - } \\
\text { Single group/cohort }\end{array}$ & 14 & 1 & 3 & 4 & 4 & 2 & \\
\hline Pathophysiology & 3 & & 1 & 1 & 1 & & \\
\hline Pediatrics & $6^{\mathrm{a}}$ & & 2 & 1 & 1 & 1 & \\
\hline Neuropsychology & 4 & & 1 & 2 & 1 & & \\
\hline Intervention & 6 & & 1 & 1 & 2 & 1 & 1 \\
\hline Review & 3 & & & & & & \\
\hline Total & 45 & 1 & 8 & 15 & 12 & 3 & 1 \\
\hline
\end{tabular}

single group design, 10 of which were moderate quality, and two were of high quality. ${ }^{24,25}$ Both of these studies were also classified as Level III. Among the single group studies, there were five rated as Level III, five rated as Level IVA, and two rated as Level V. The use of a comparison group is a stronger design; however, our quality rating scheme studies included several elements, weighted equally, to assess overall quality so that a study weak in one area can be strong in other areas.

There are several important elements that should be addressed in a good quality epidemiological study. Recruitment is a key issue and recruitment methods must be carefully considered to make sure that any selection bias is eliminated. Recruitment from the perspective of time postinjury is also a factor for consideration in that it allows us to observe the developmental time course of the disorder. Among the articles we reviewed, $65 \%$ of the studies $(15 / 23)$ used consecutive recruitment; however, only $13 \%$ (3/23) used consecutive recruitment directly from the acute setting. Twenty-six percent were consecutively recruited as patients entered rehabilitation and the remaining $26 \%$ were recruited while in rehabilitation or upon discharge from rehabilitation. Parcell and colleagues ${ }^{26}$ recruited all patients with TBI, with no preference for those with sleep complaints, thus reducing bias in their sample. The remainder of the studies included retrospective chart review or recruitment by advertising. In contrast to the Parcell study, the study by Ouellet and colleagues, ${ }^{4}$ with the largest sample size of 452, recruited participants with reported symptoms of insomnia via mailings sent to 1500 prospective participants from archives of Québec City's largest rehabilitation center, local brain injury associations, and support groups. The authors specifically reported using liberal inclusion criteria to try and capture a "broad portrait of the TBI population." 4 (p. 201)

It is also important to consider possible confounders or variables that influence interpretation of the findings, such as preexisting, concurrent, and secondary comorbidities; age at onset of injury; time since injury; severity of injury; etiology of injury; and gender. In an "ideal" study, the sample population would be large enough to allow stratification on some or all of these variables, so that the findings would be more generalizable and create a more thorough picture of the phenomenon.

Overall, the objective of the epidemiological studies reviewed for this article was to document the incidence, prevalence, and nature of sleep disturbances following TBI, primarily "insomnia" per se, with the inclusion of the most recent data relating specifically to sports-related concussion. 


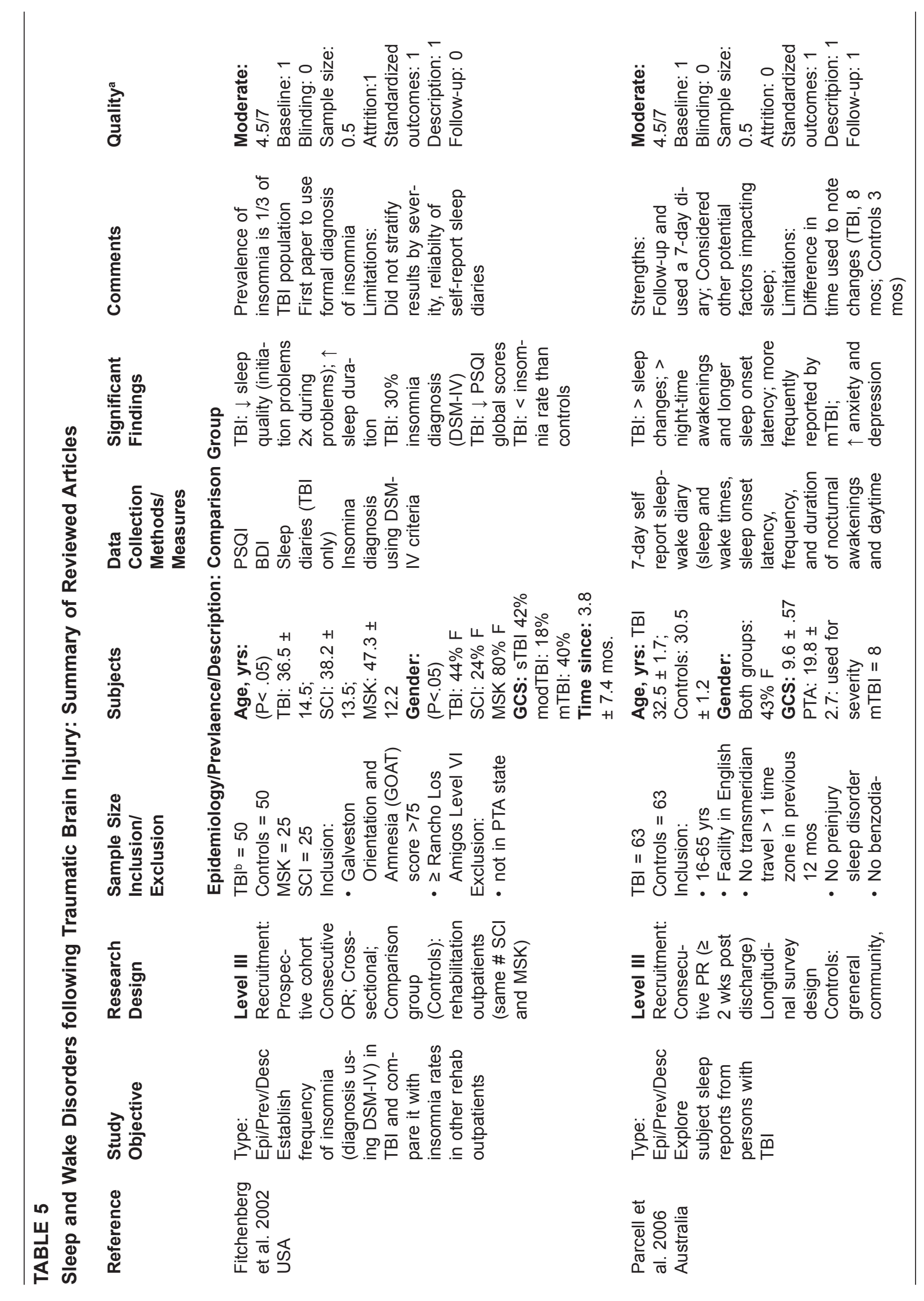




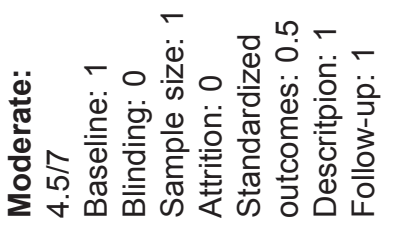

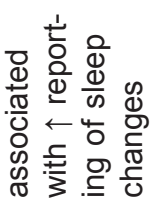

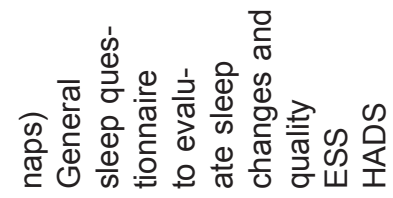

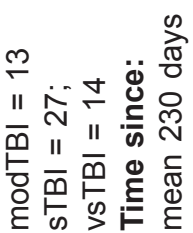

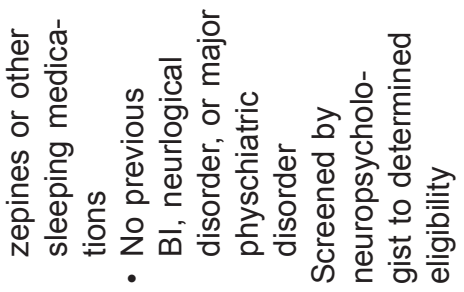

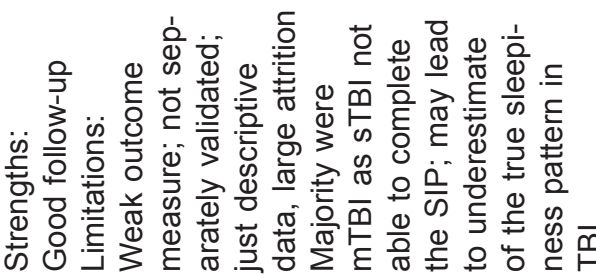

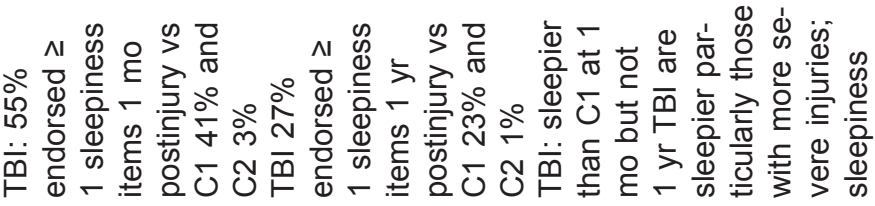

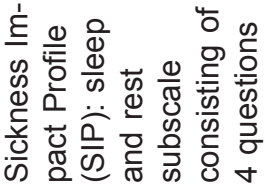

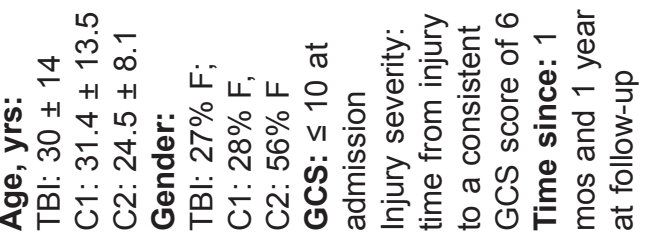

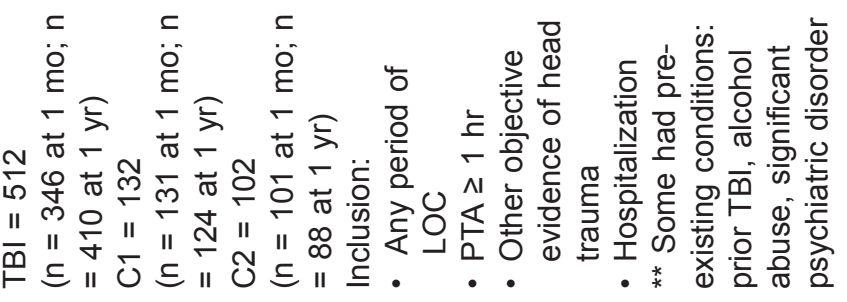

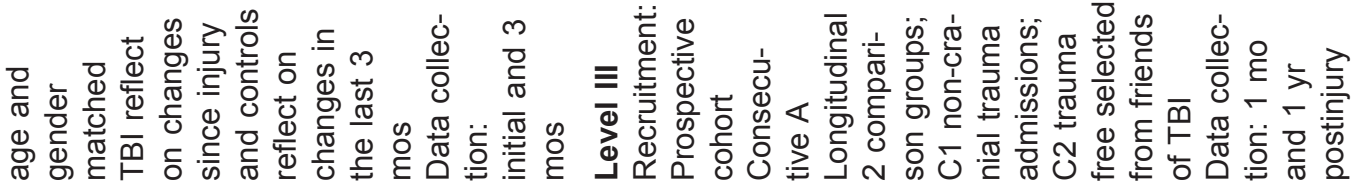

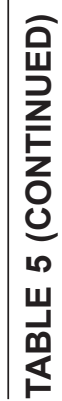
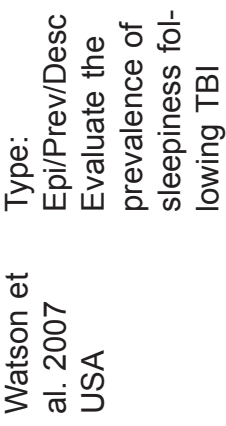

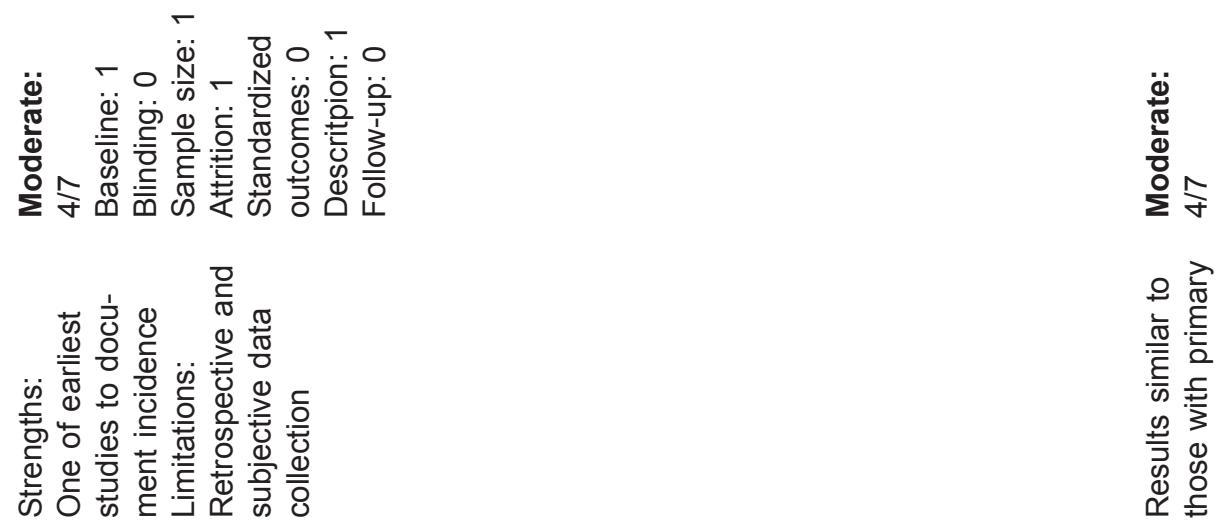

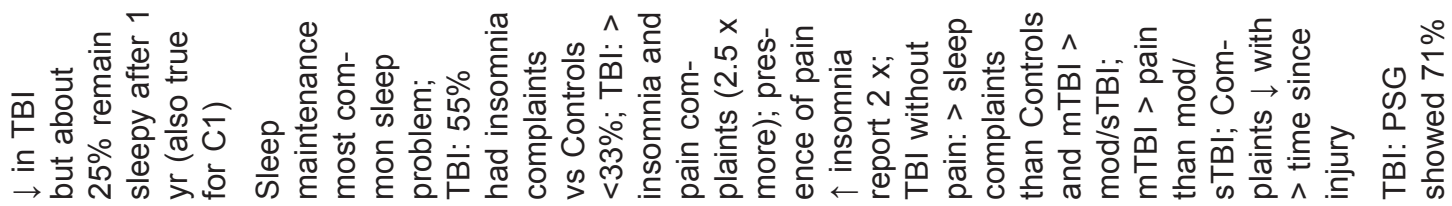

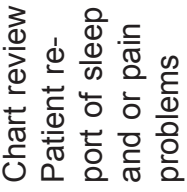

은

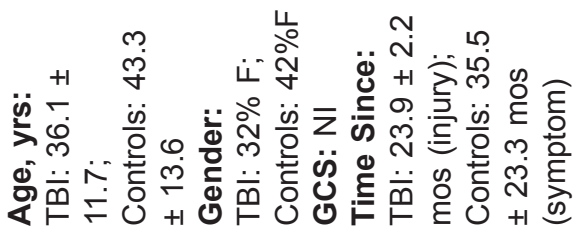

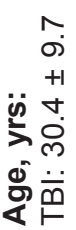

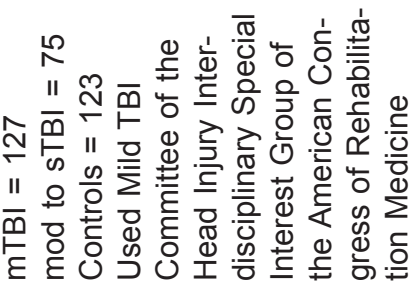

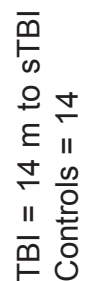
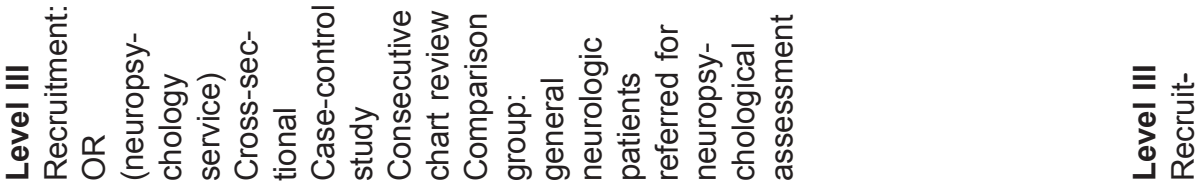

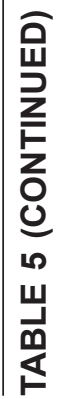

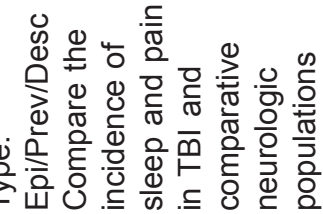

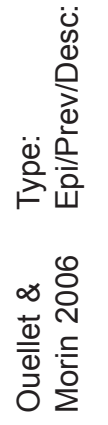




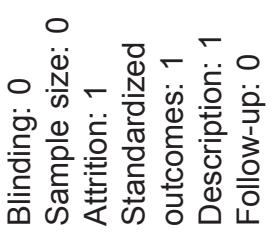

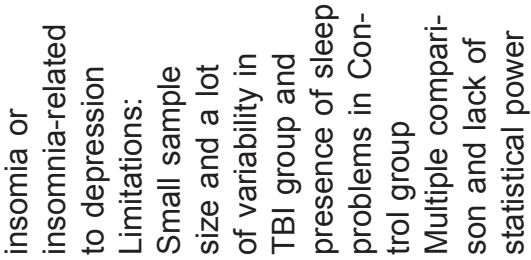

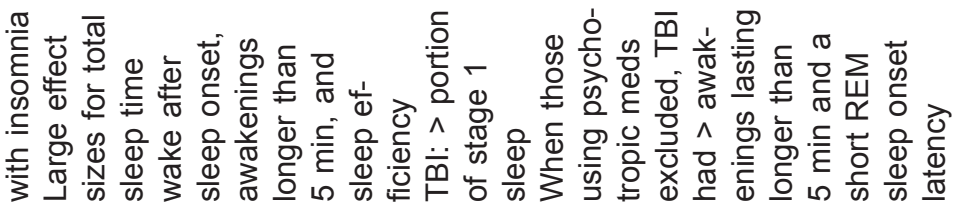

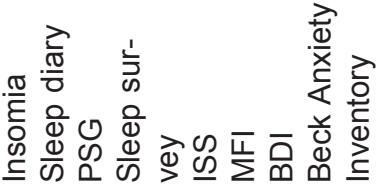

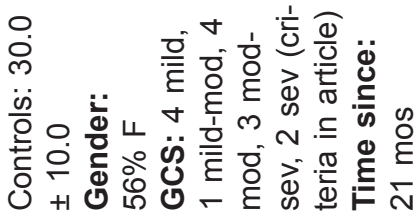

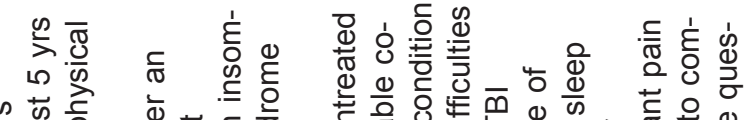

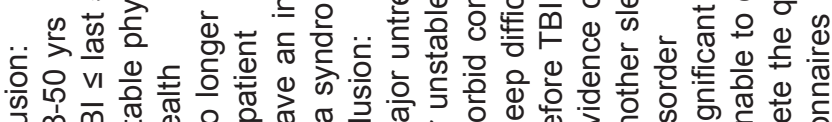

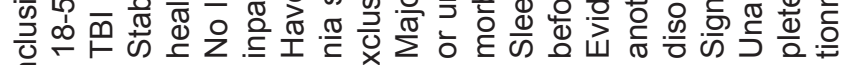

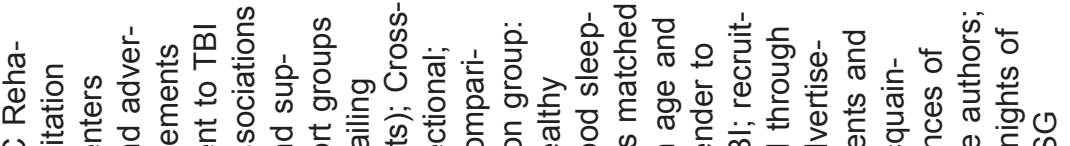

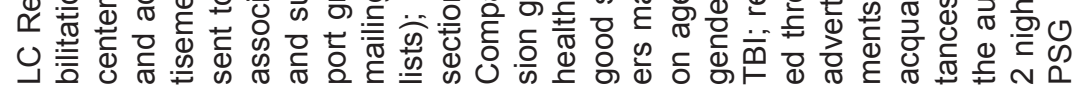

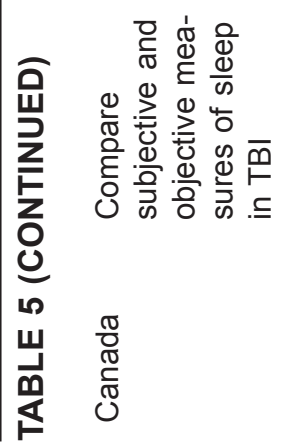

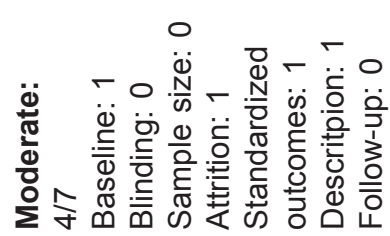

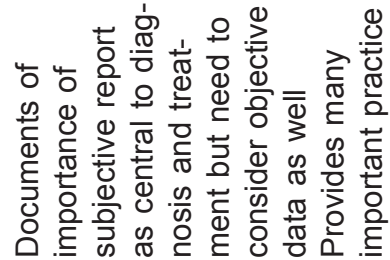

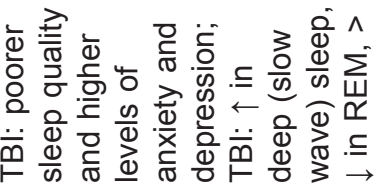

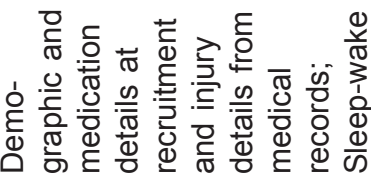

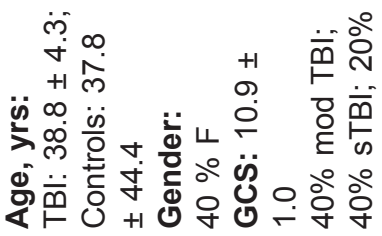

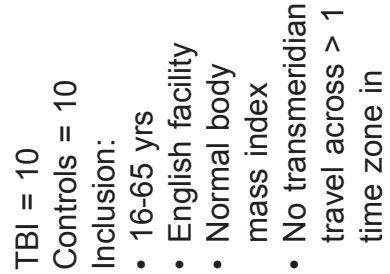

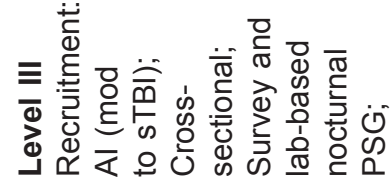

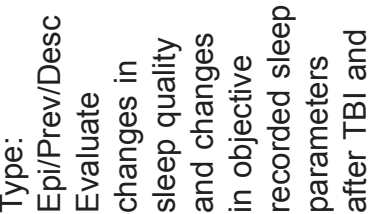

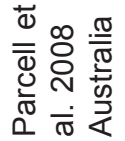




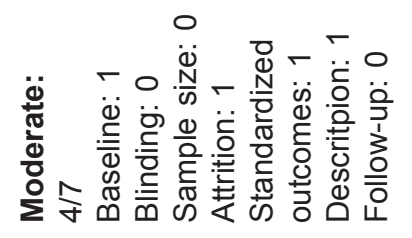

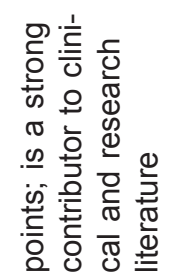

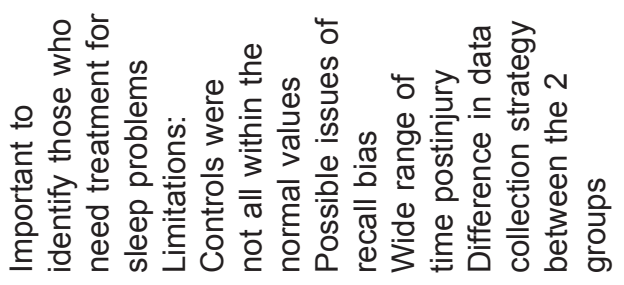

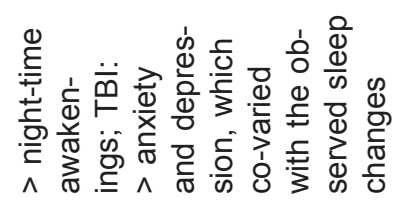

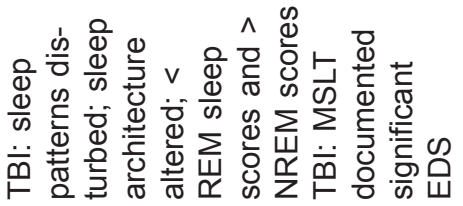

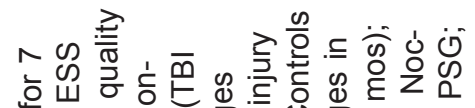

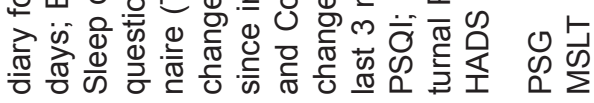

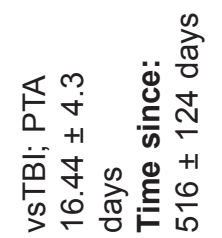

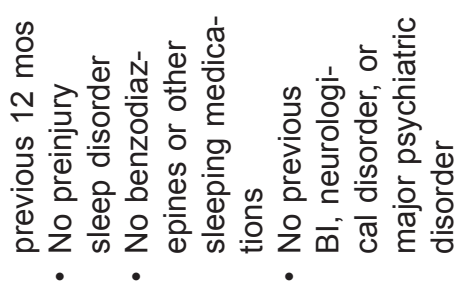

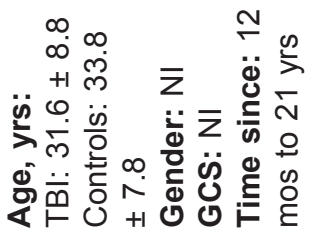

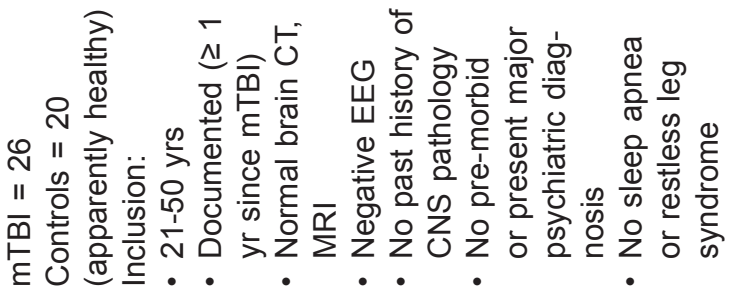

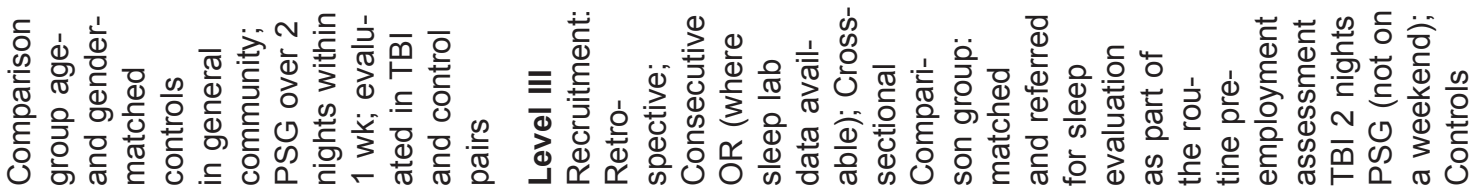

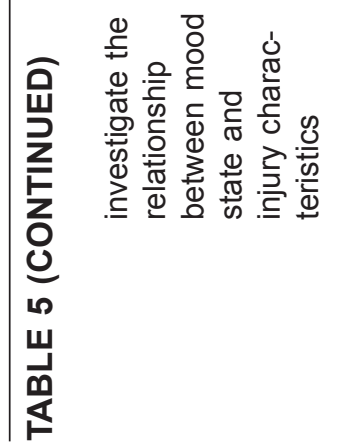




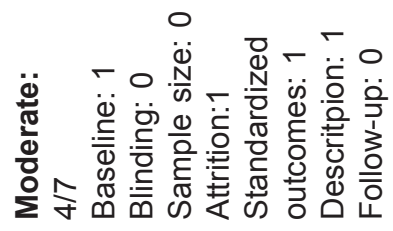

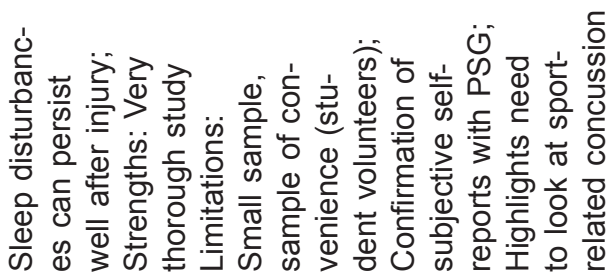

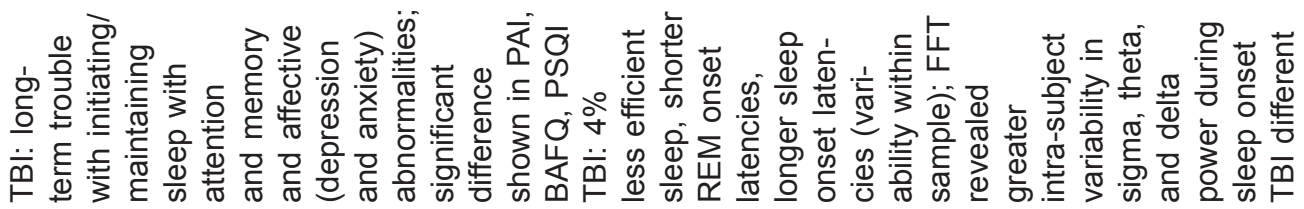

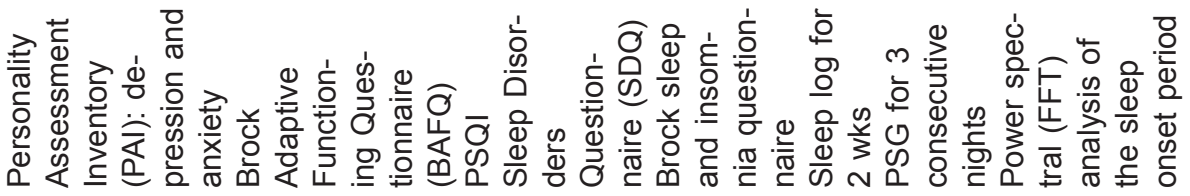
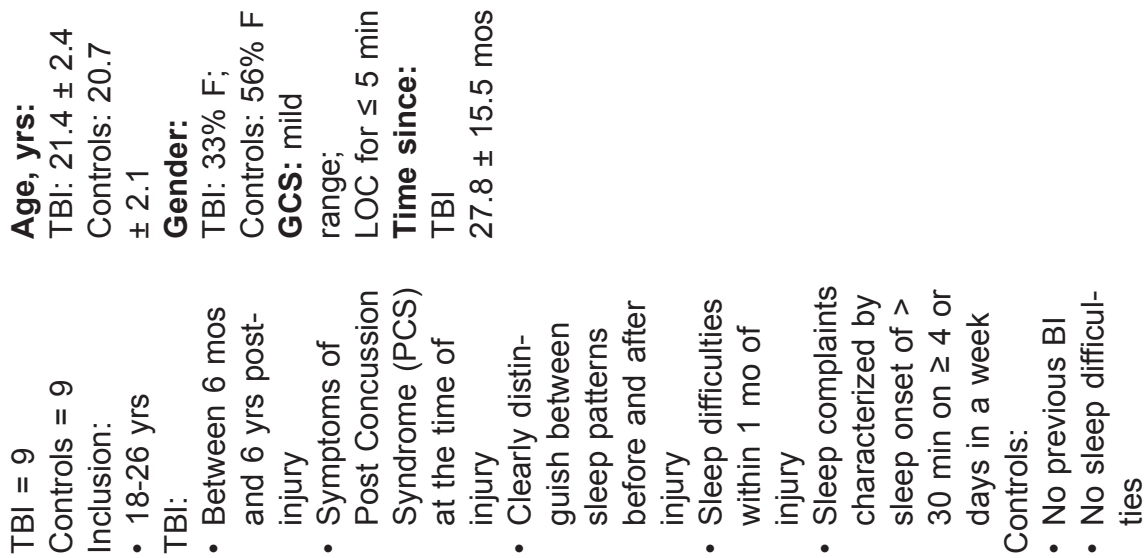

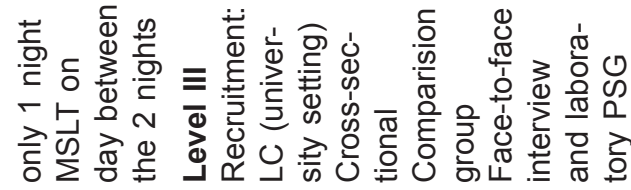

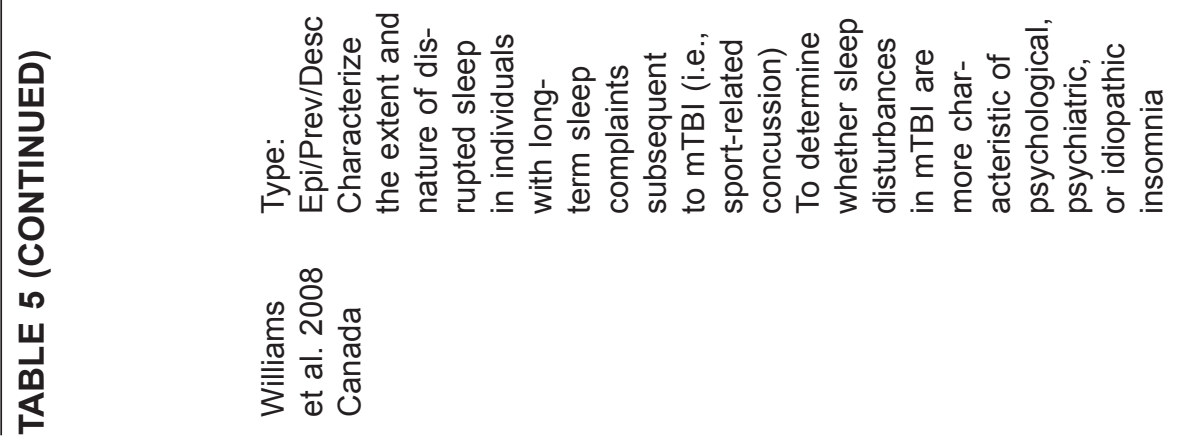



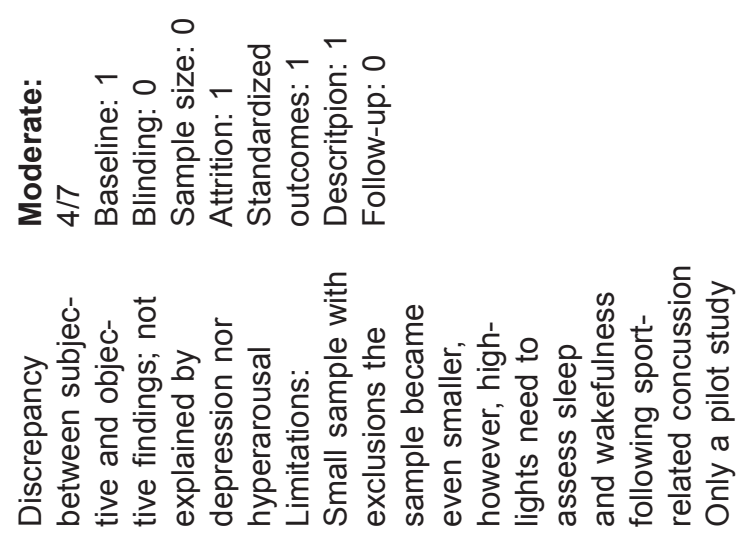

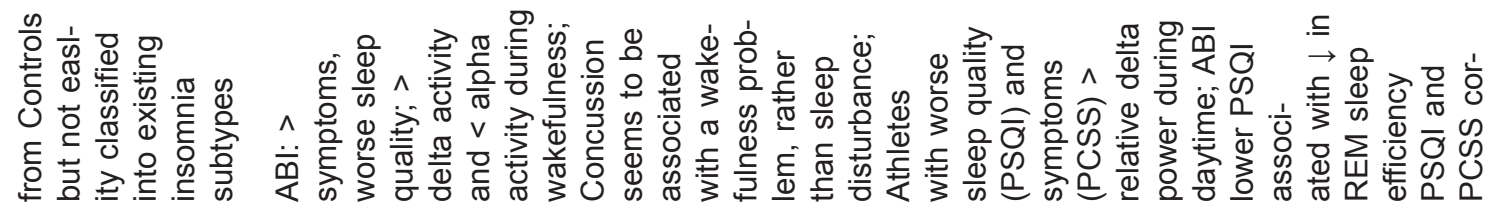

is (1) 它

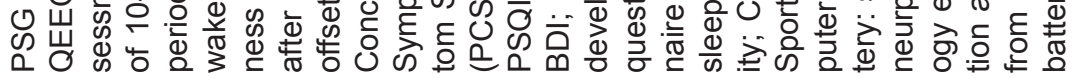
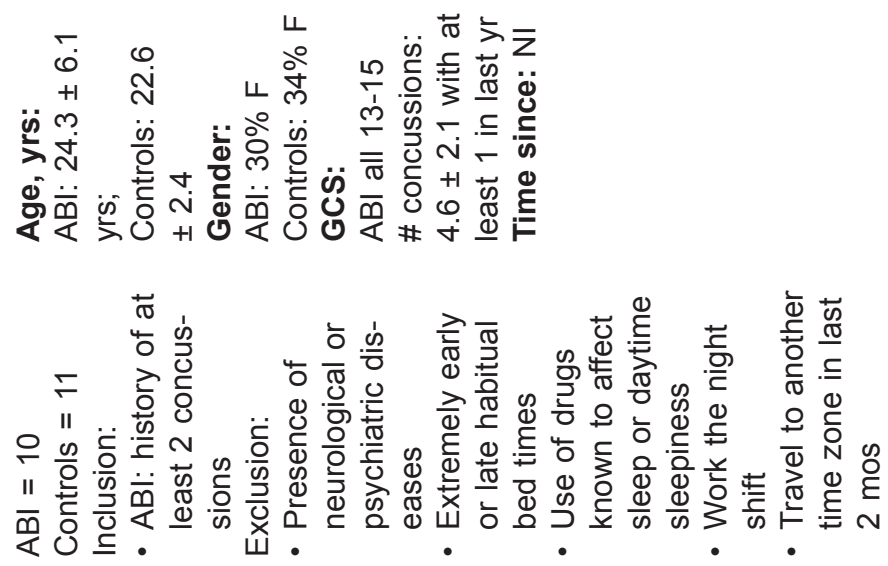

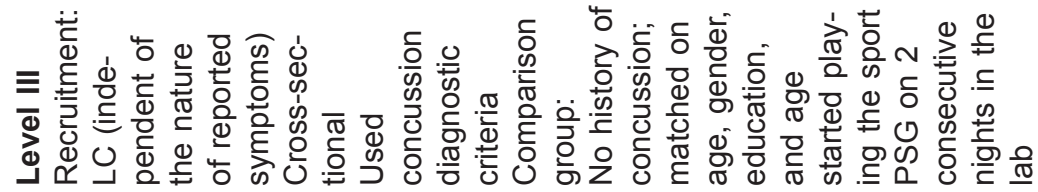

岂

$$
\begin{aligned}
& \text { ஜ }
\end{aligned}
$$

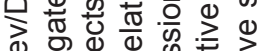

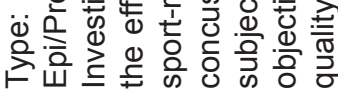

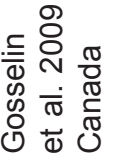




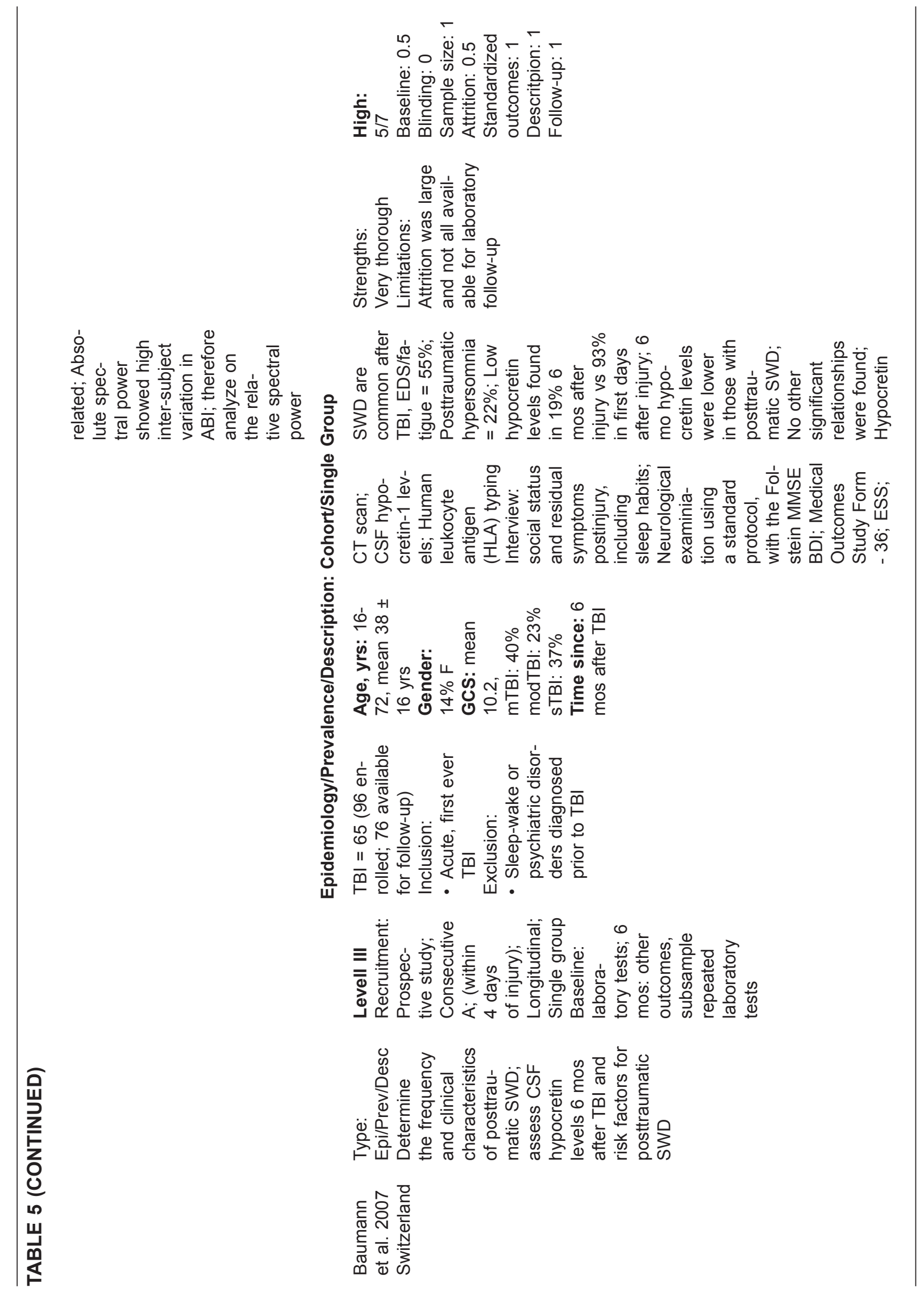




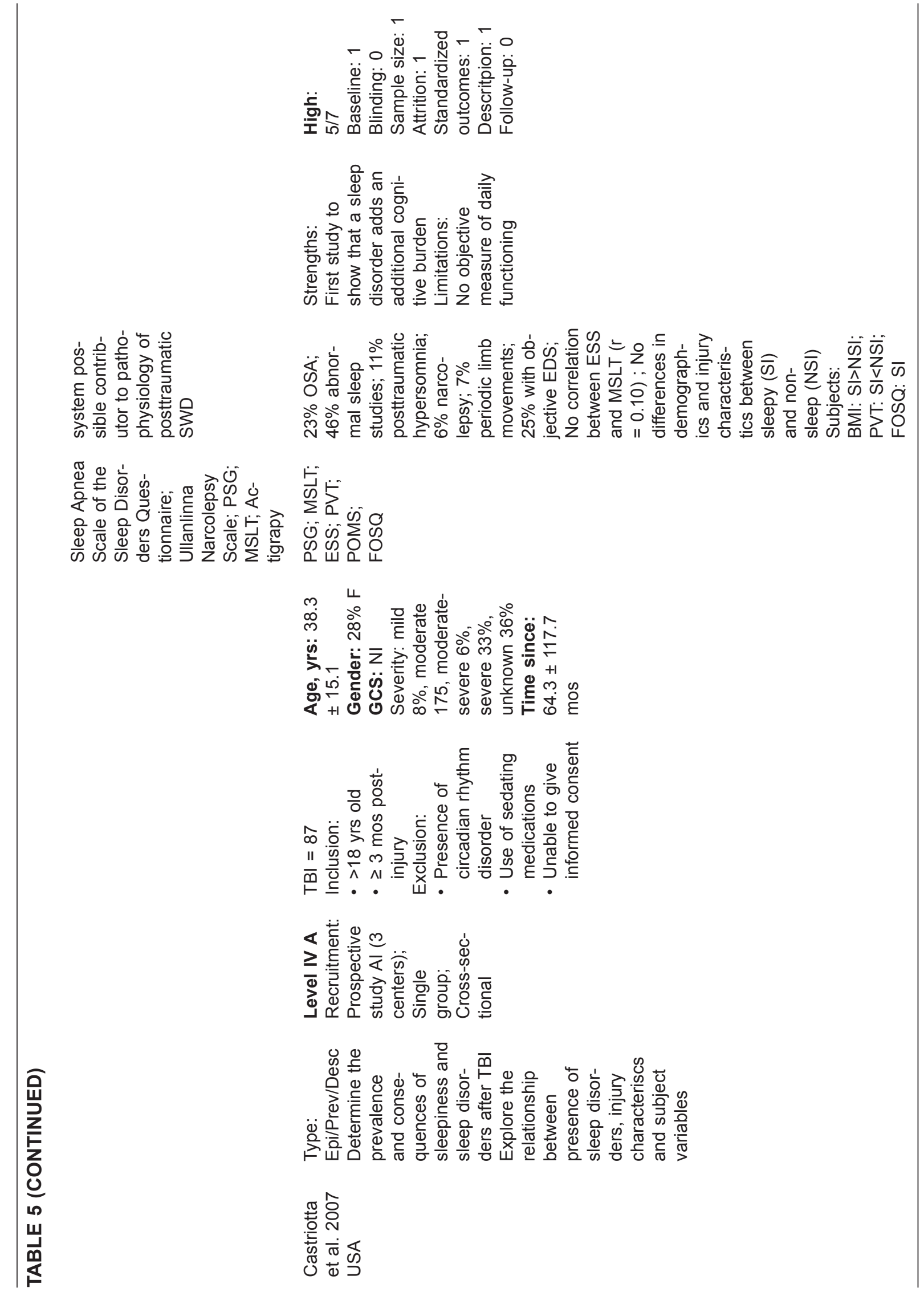



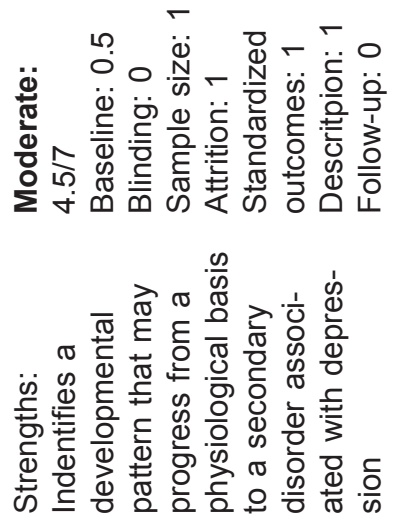

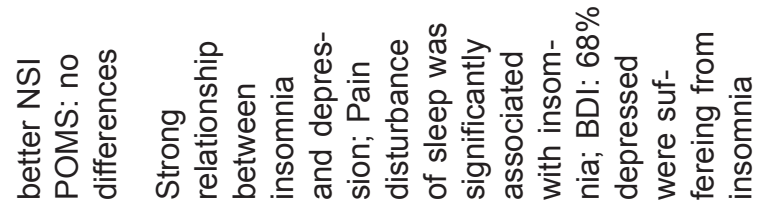

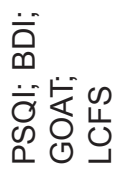

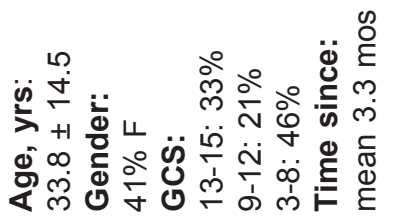

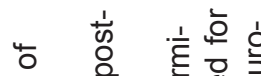

ᄂ

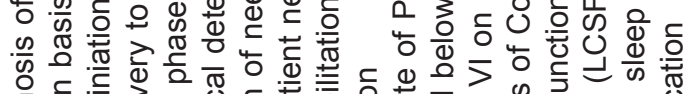
б.

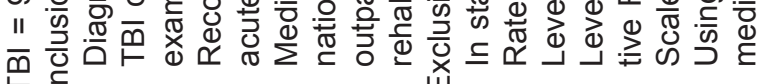
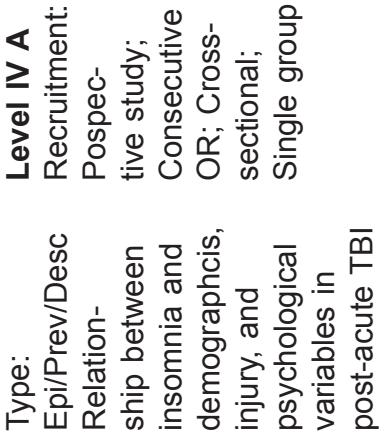

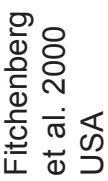

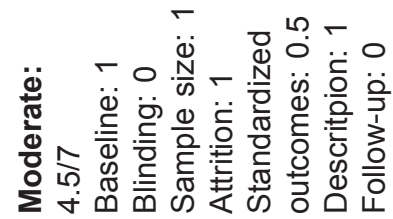

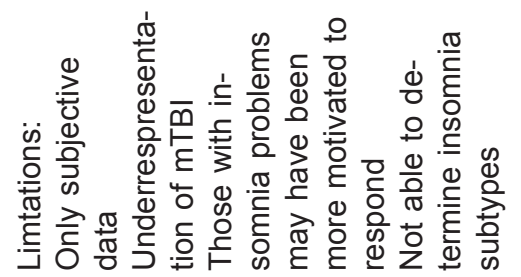

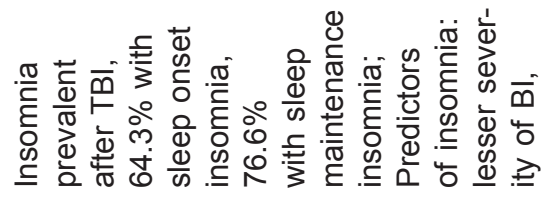

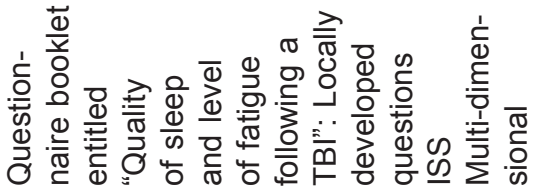

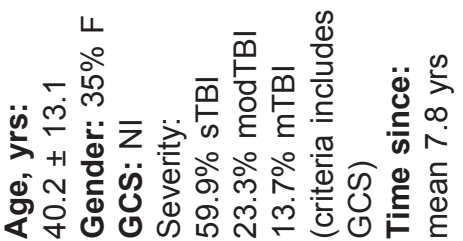

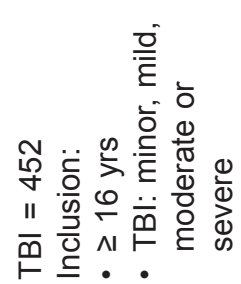

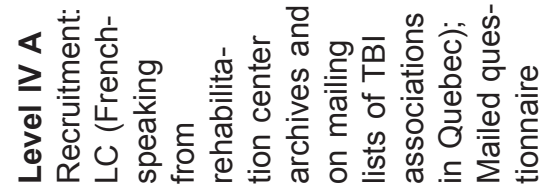

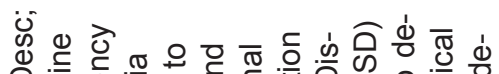
只

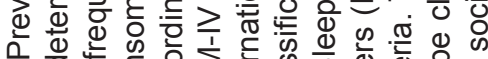

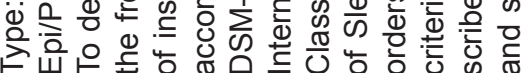

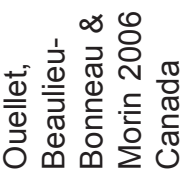




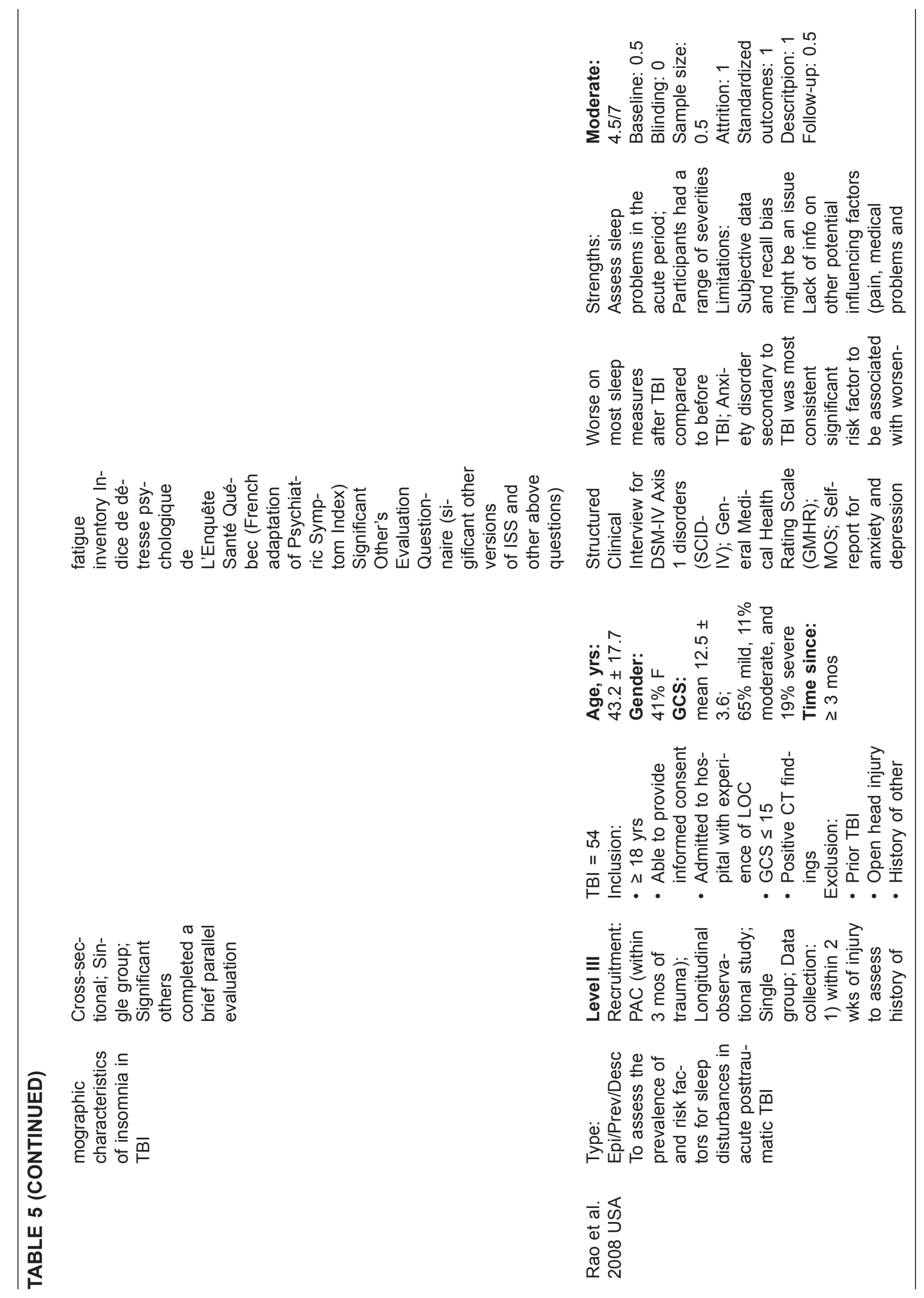




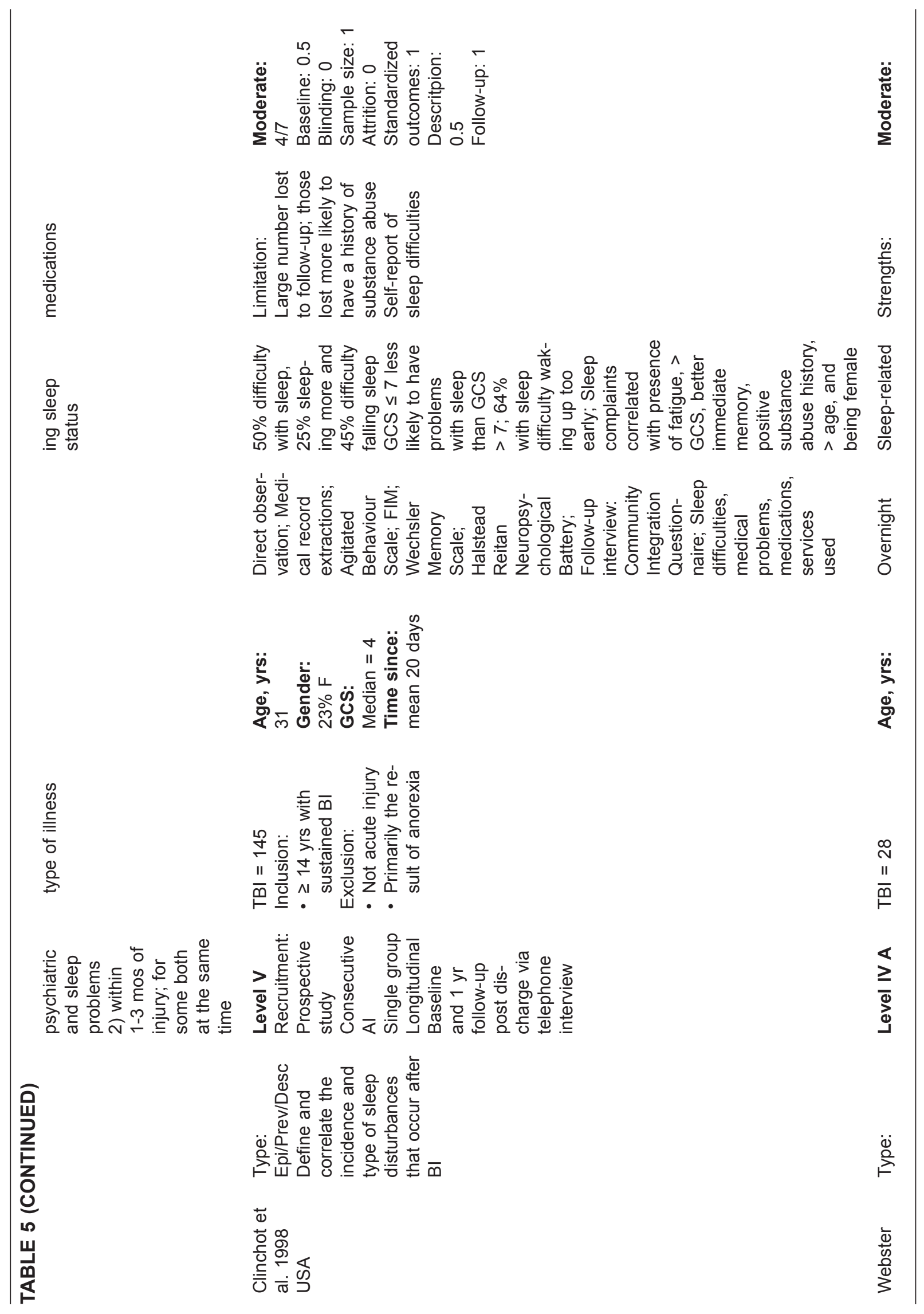




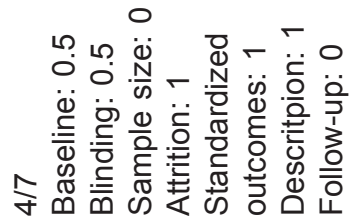

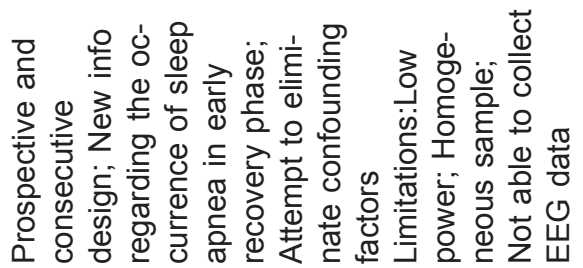

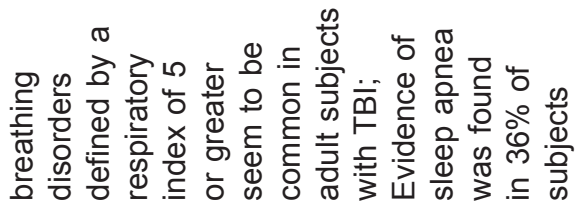

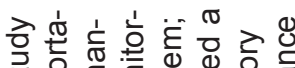

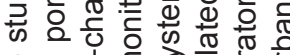

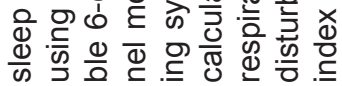

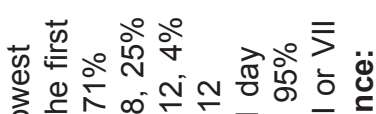

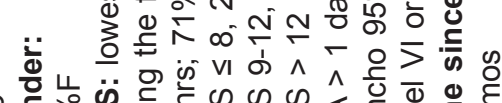

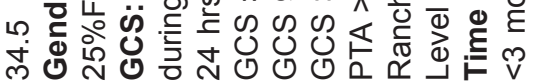

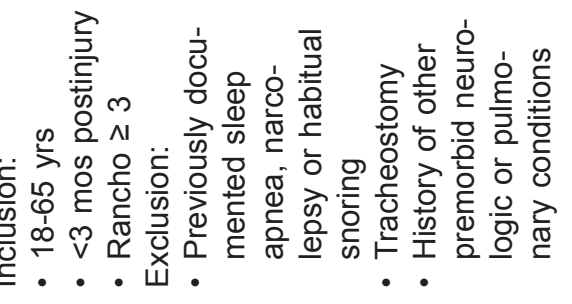

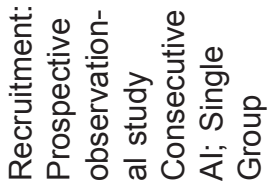

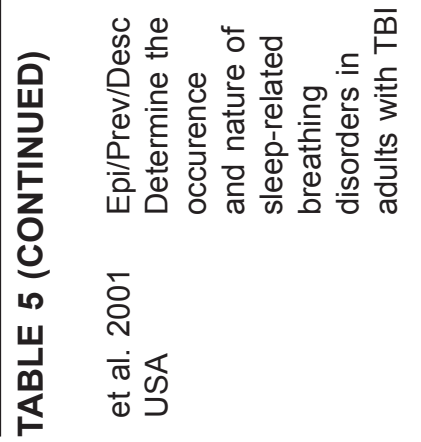

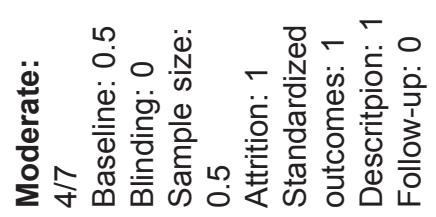

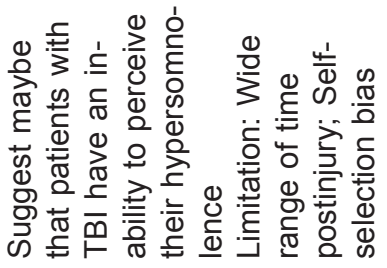

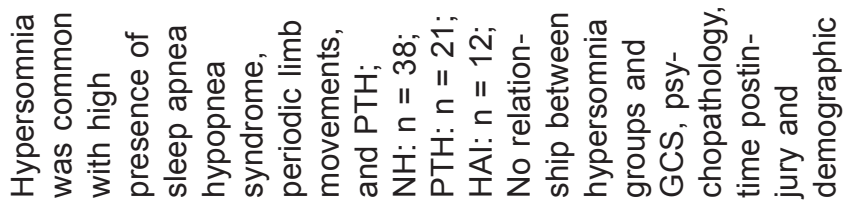

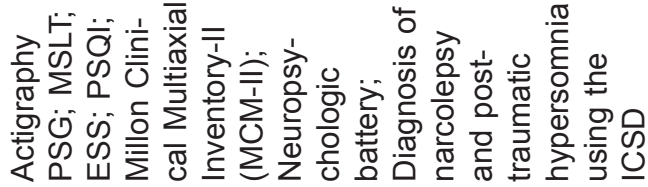

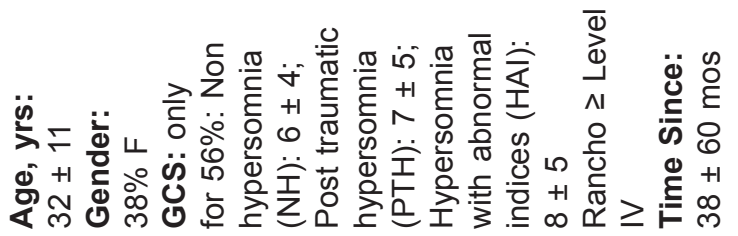

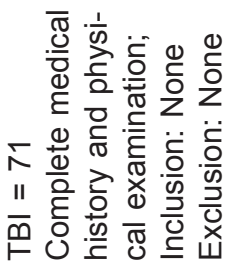

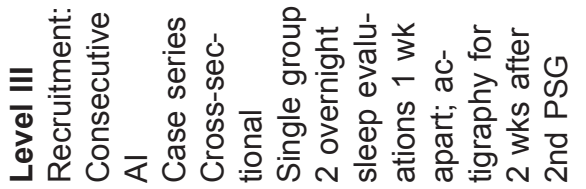

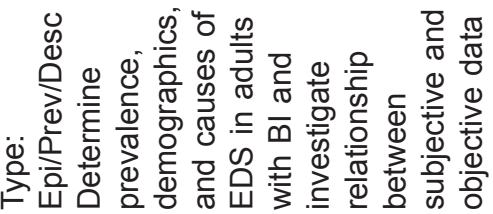

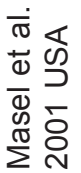




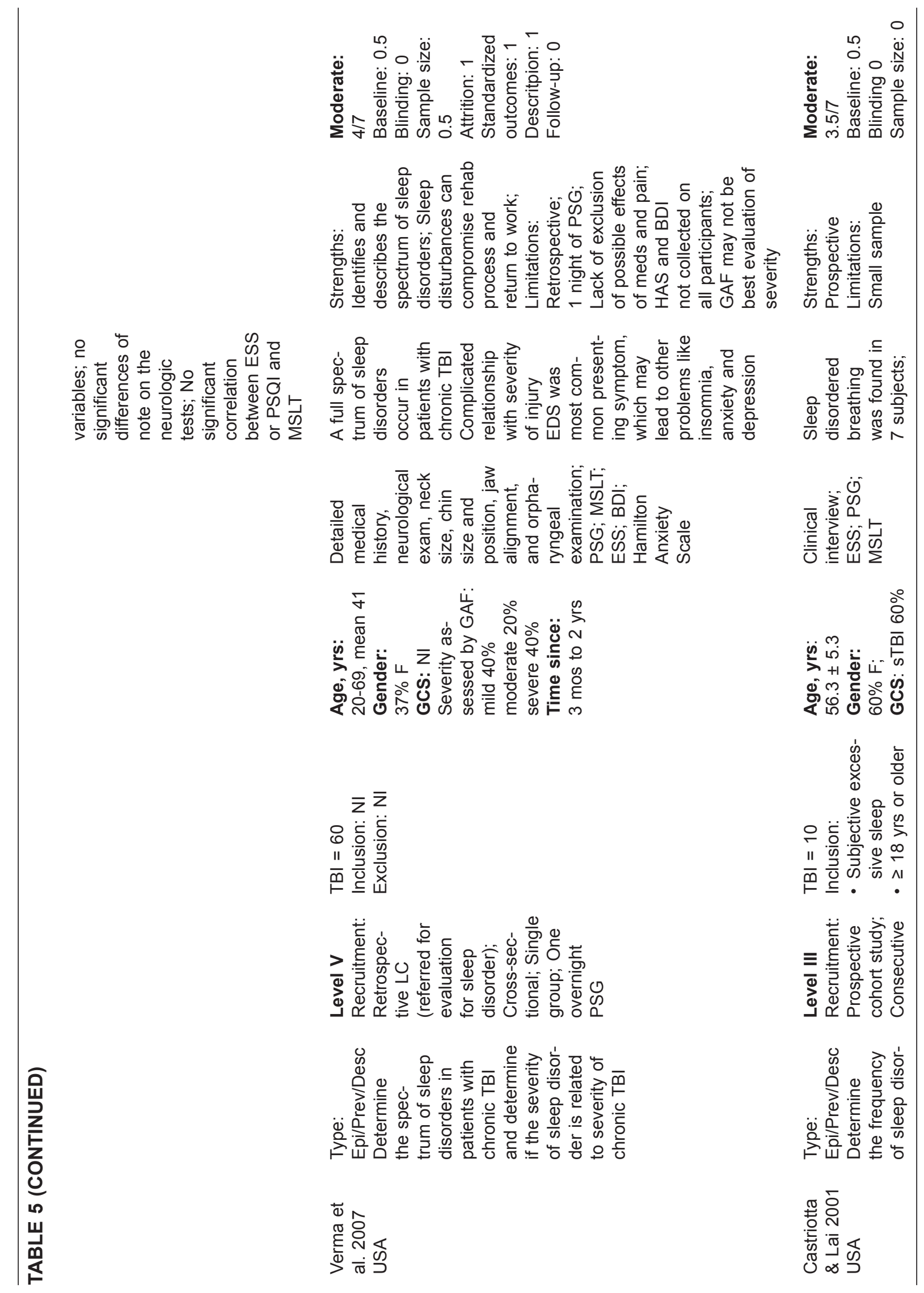




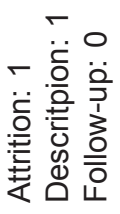
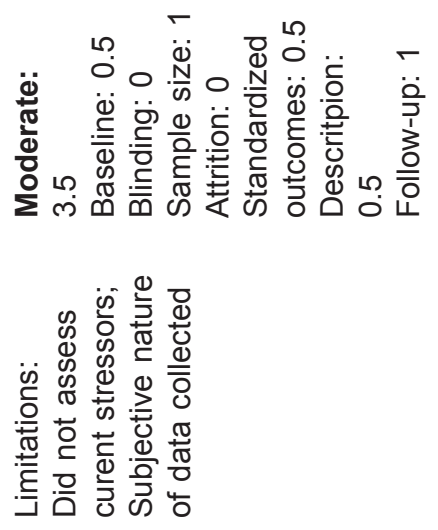

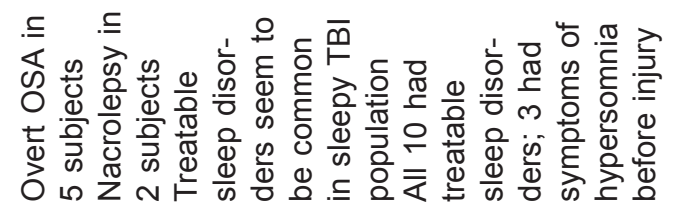

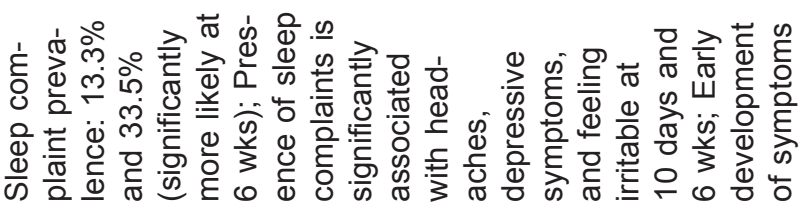

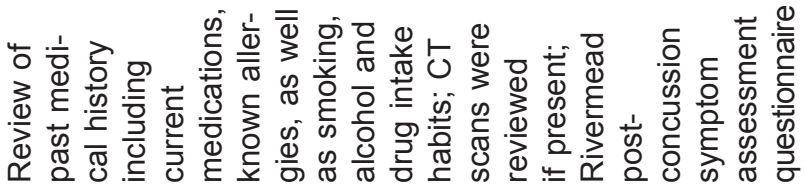

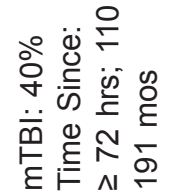

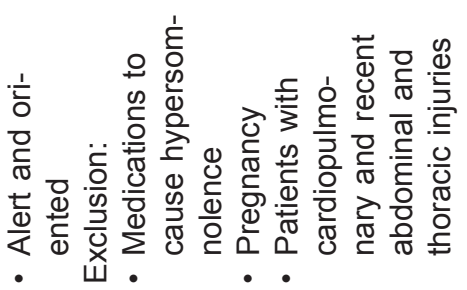

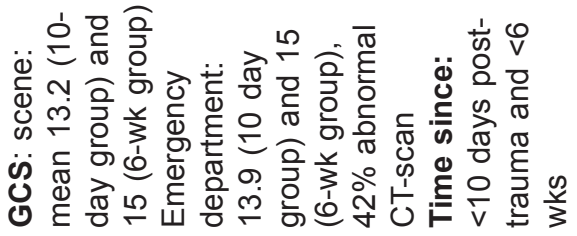

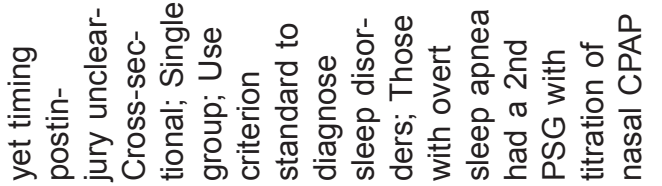

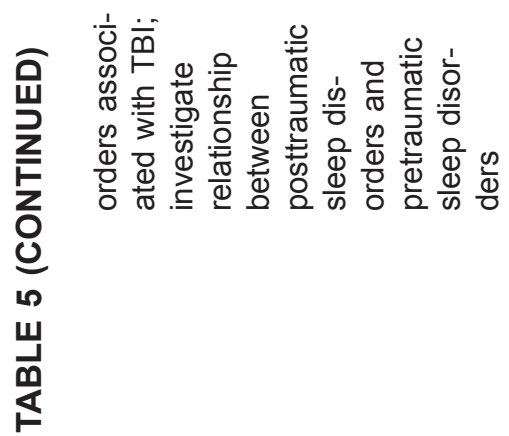

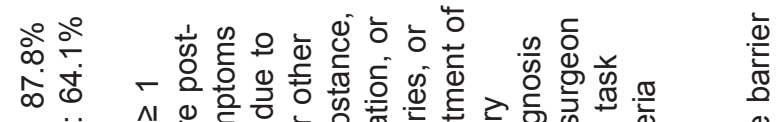

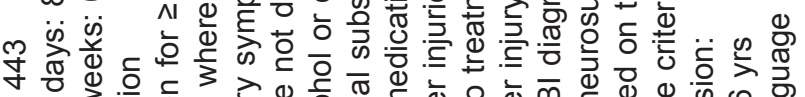

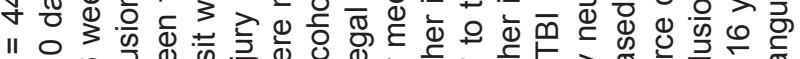

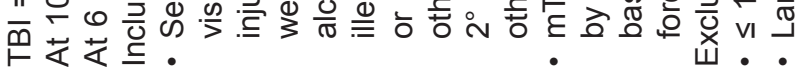

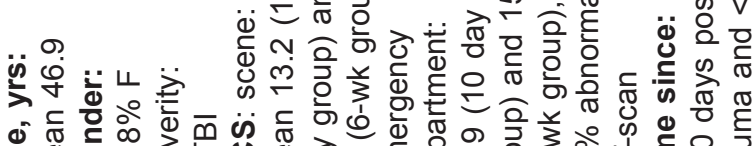

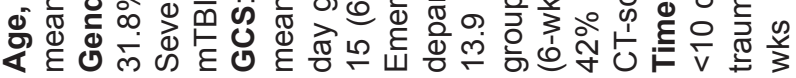

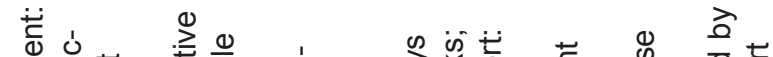

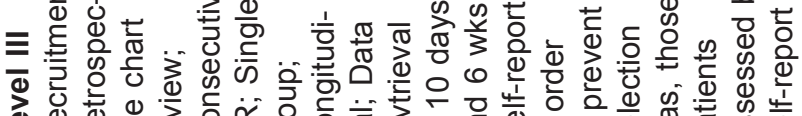

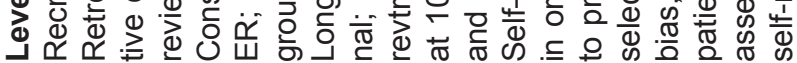

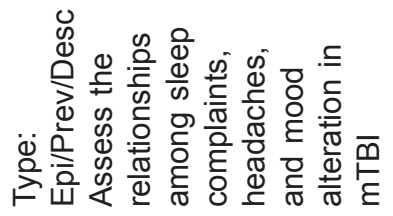

蒙 $\frac{\frac{\pi}{0}}{\frac{\pi}{\pi}}$ 

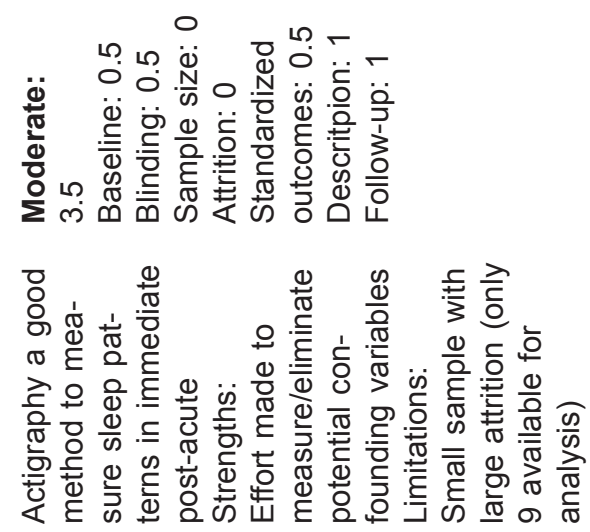

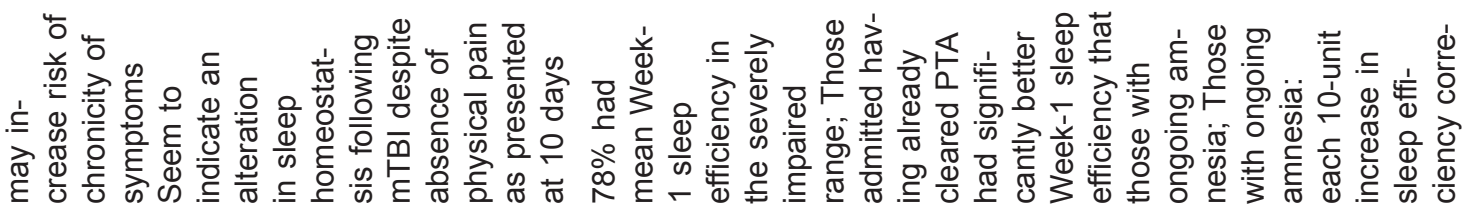

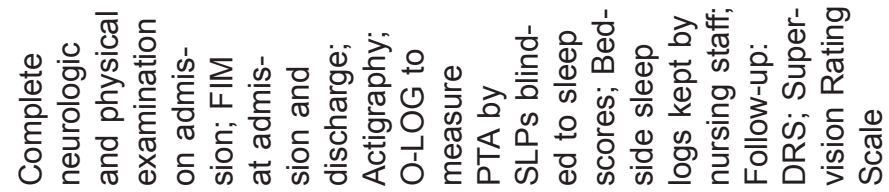

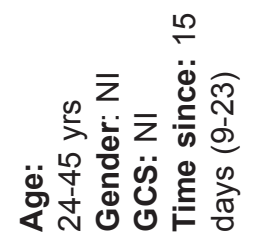

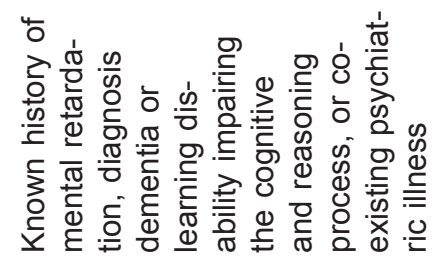

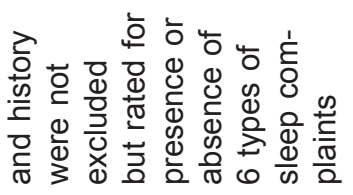

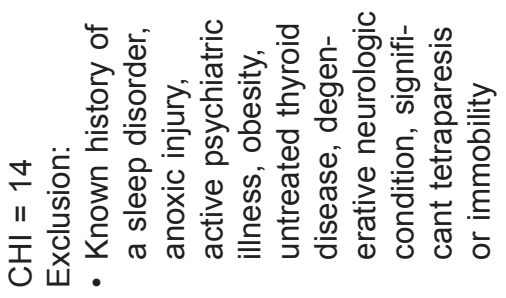

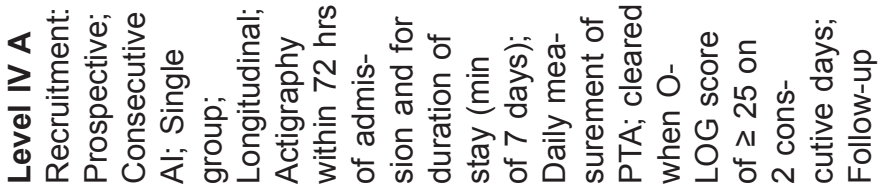

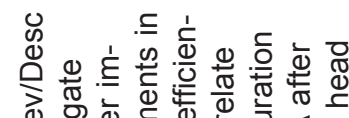

む.

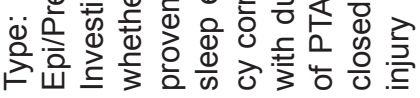

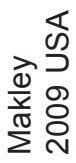



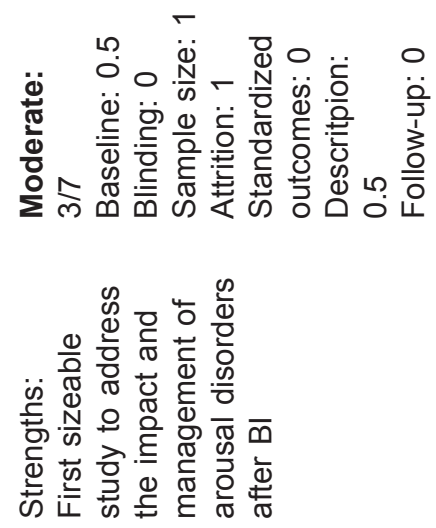

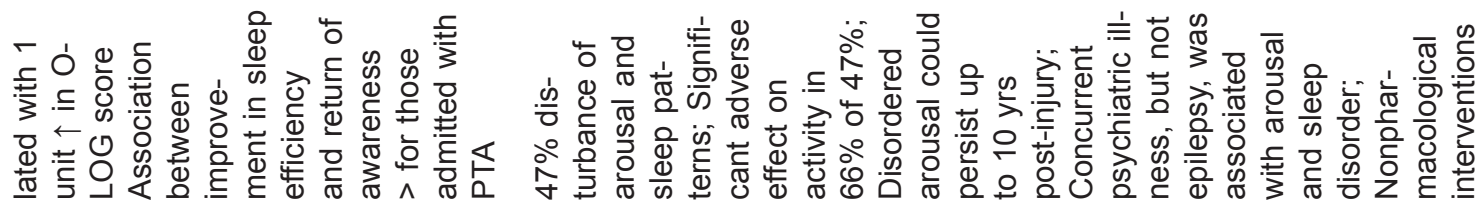

$\sum_{\omega}^{\infty} \overline{0}$

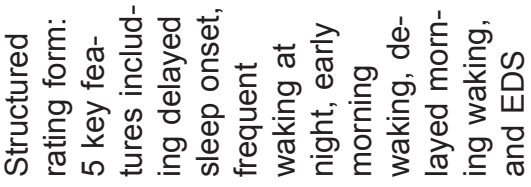

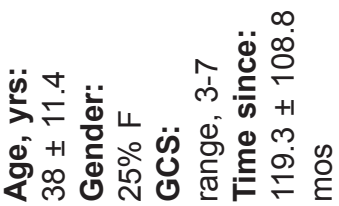

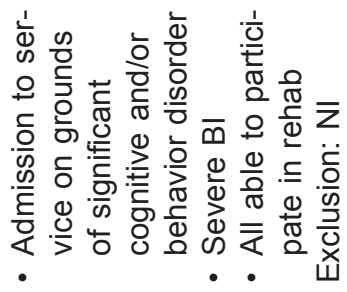

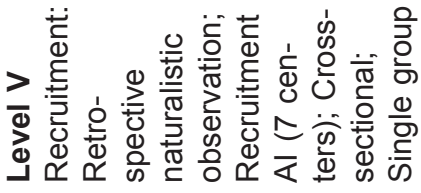

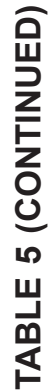

呬

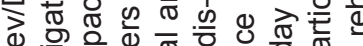

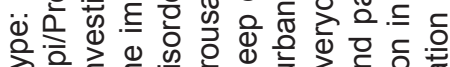

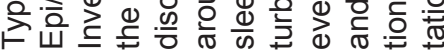

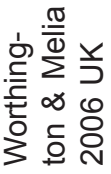




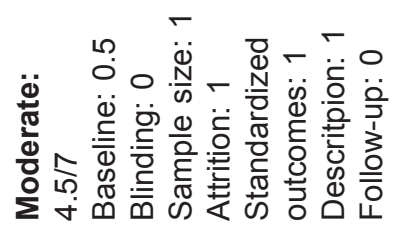

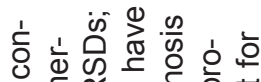

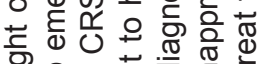

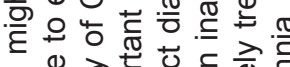

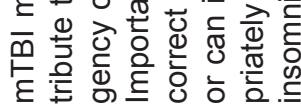

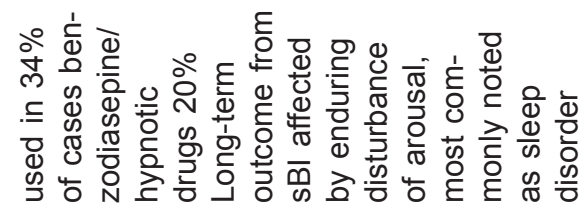

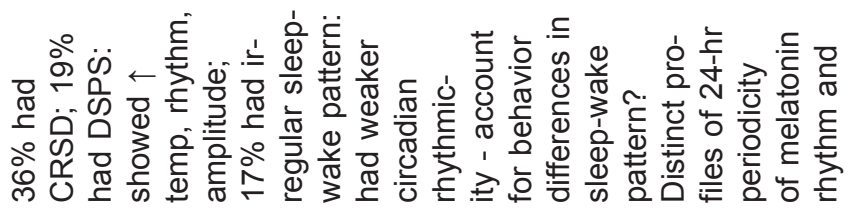

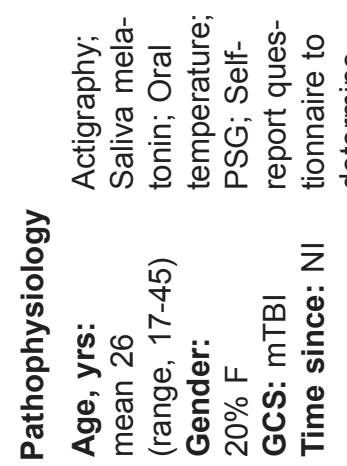

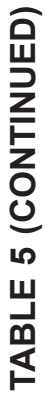

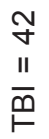
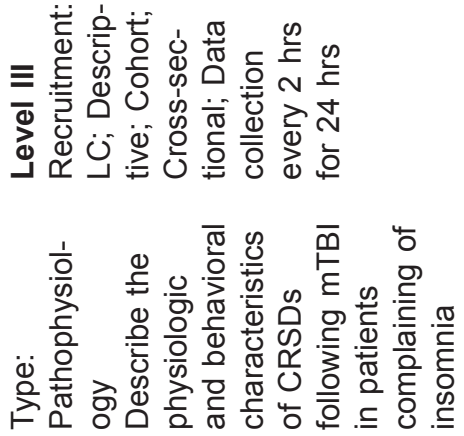

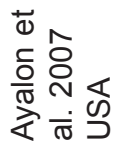



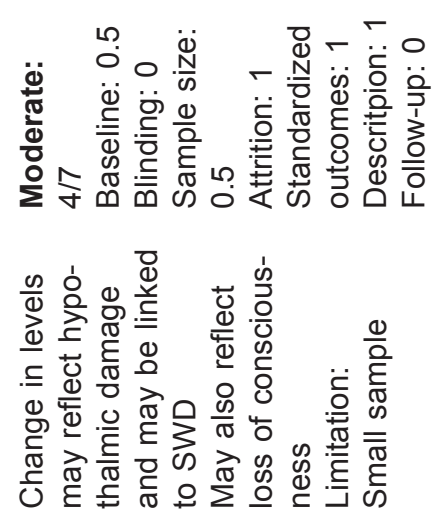

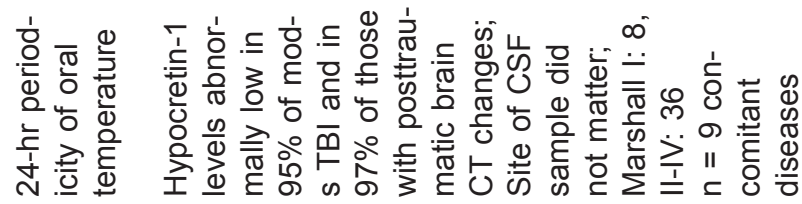
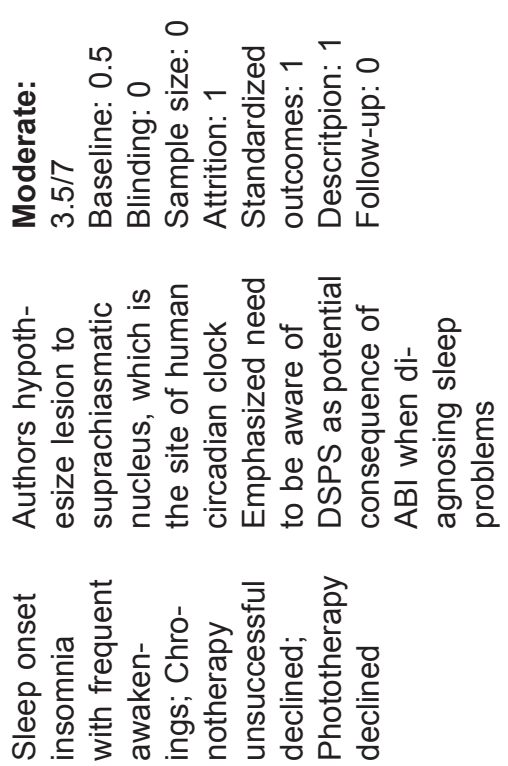

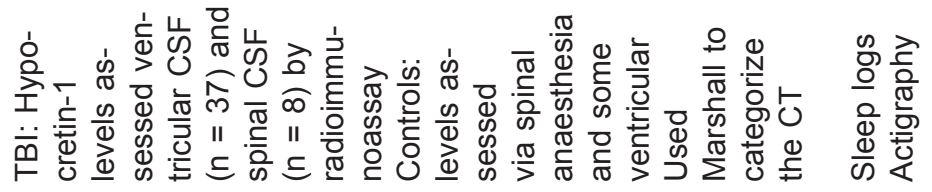

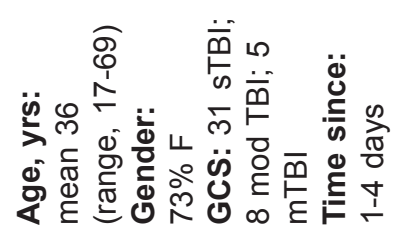

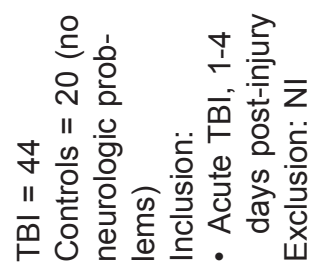

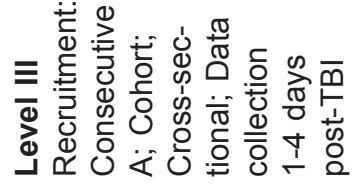

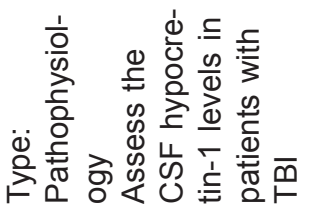

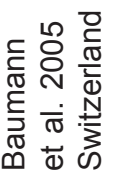

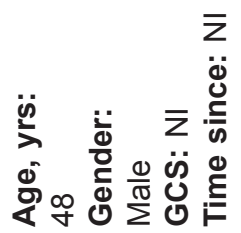
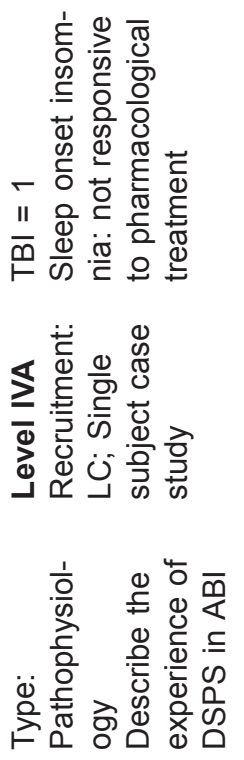

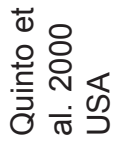




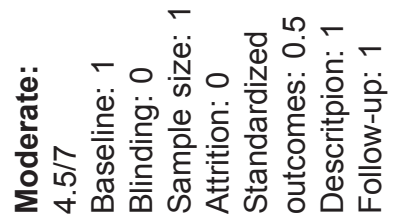

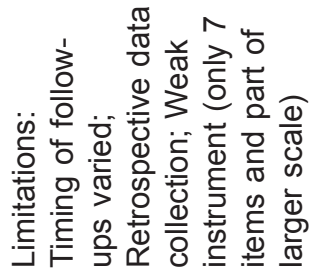

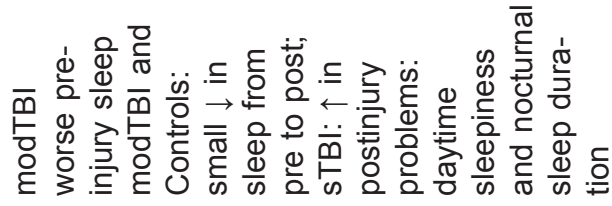

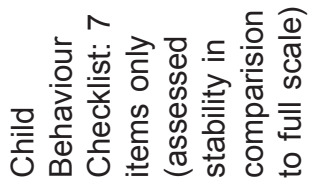

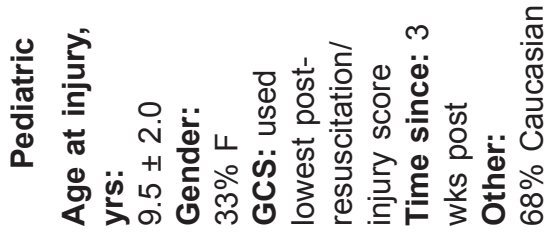

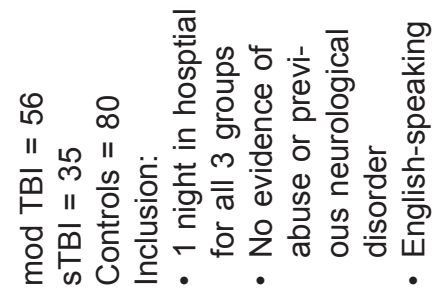

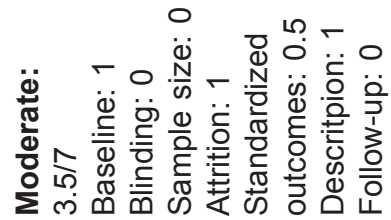

¿

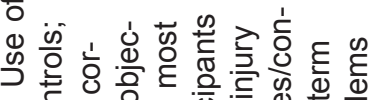

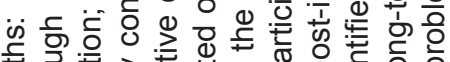

易

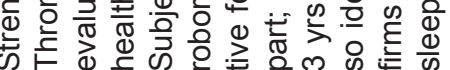

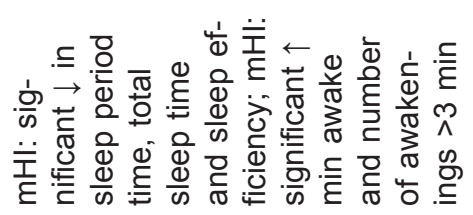

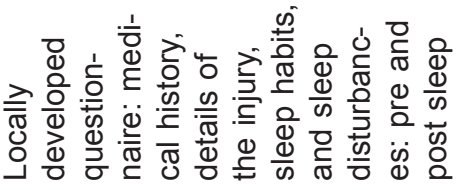

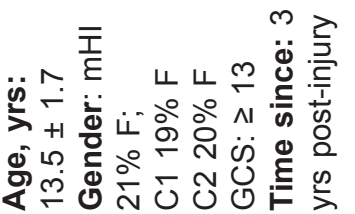

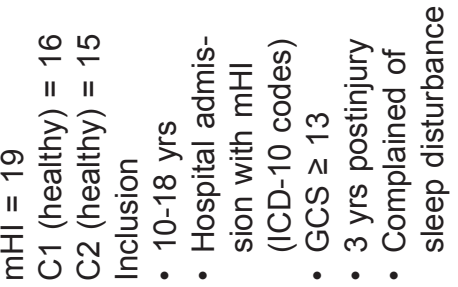

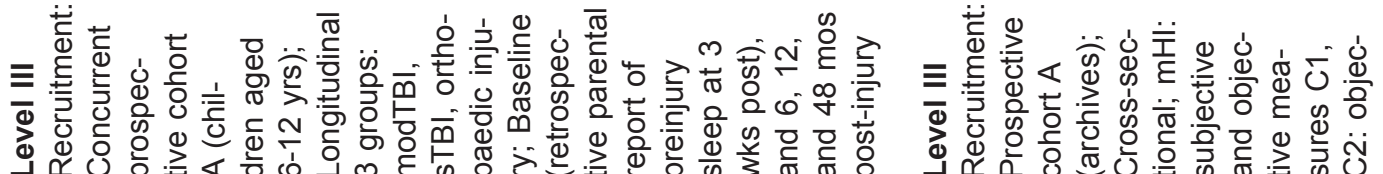

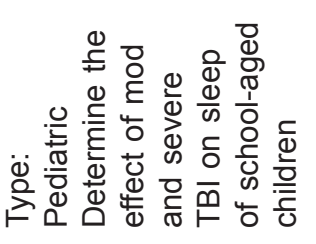

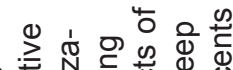

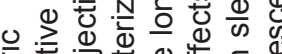

‥

ฑั

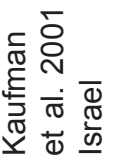



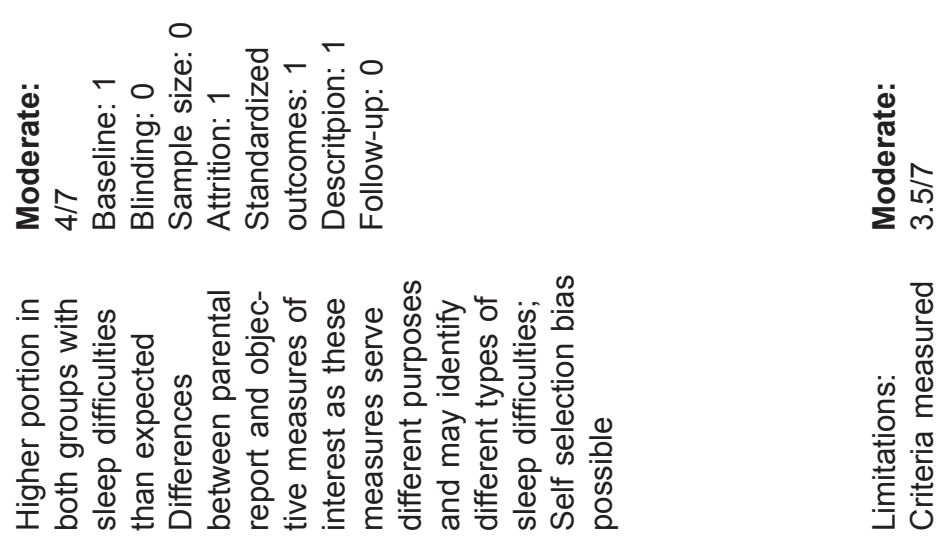

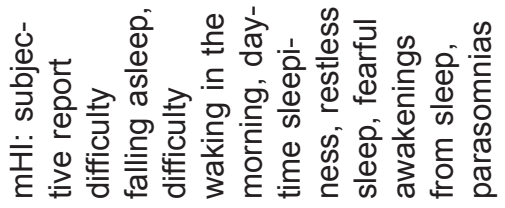

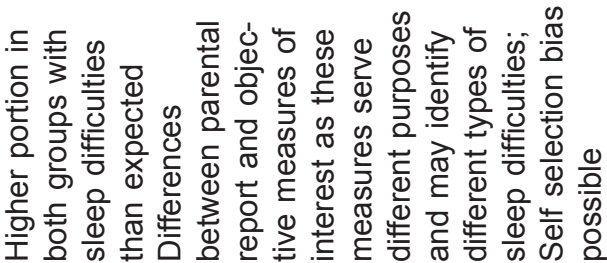

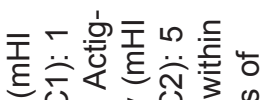

仓

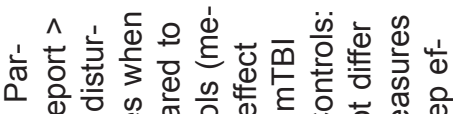

‥

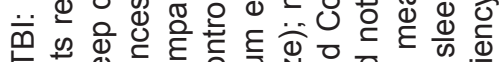

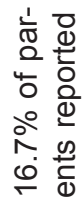

व क

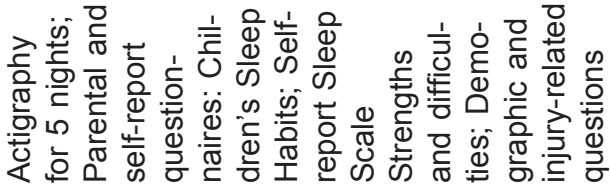

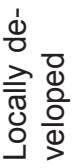

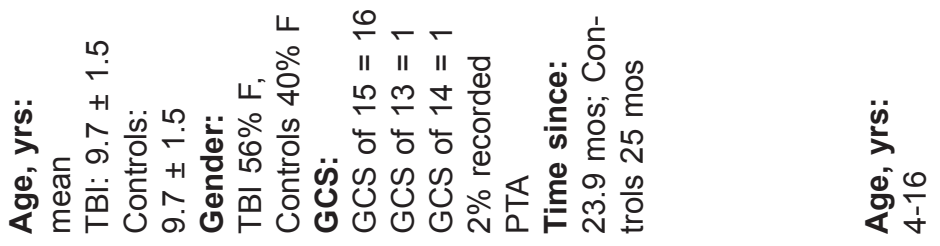

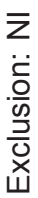

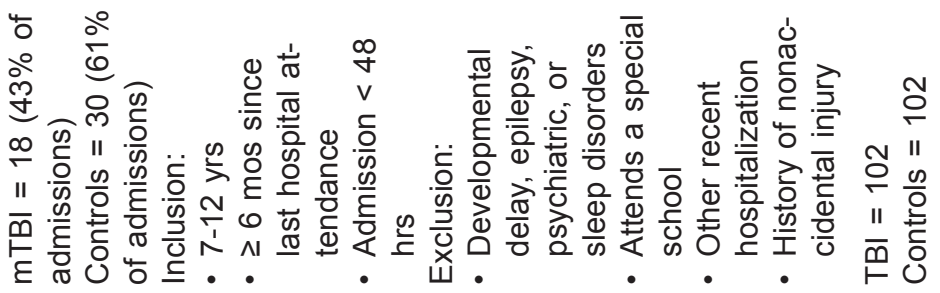

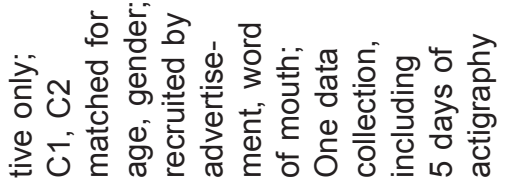

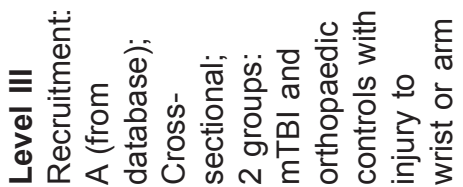

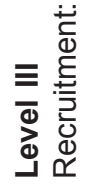

吕

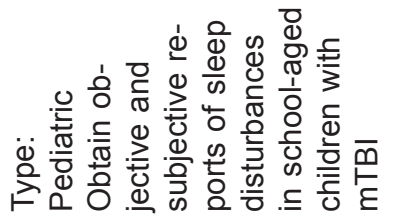

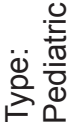

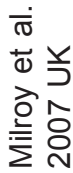

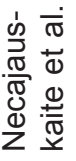




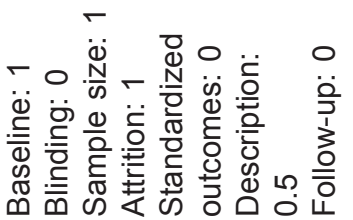

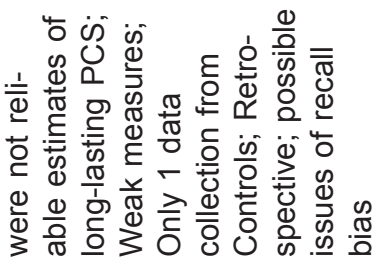

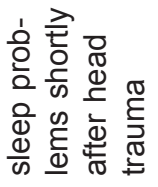

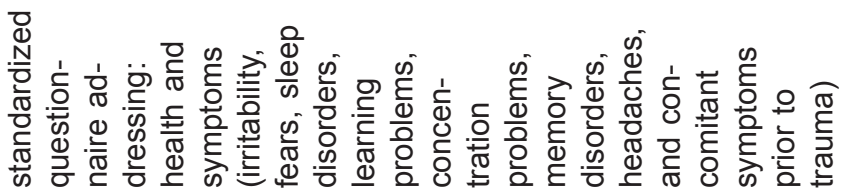

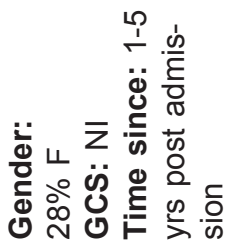

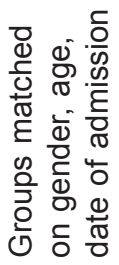

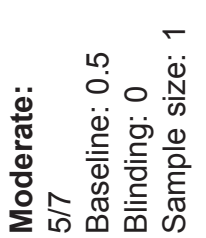

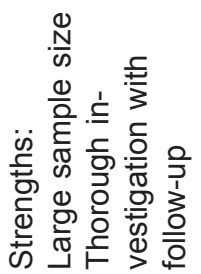

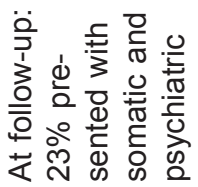

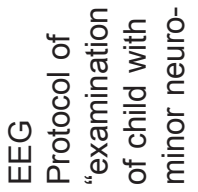

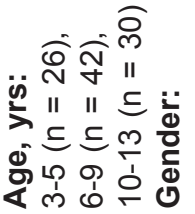

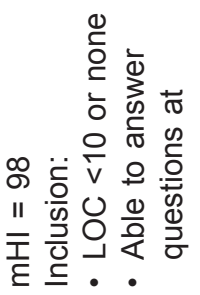

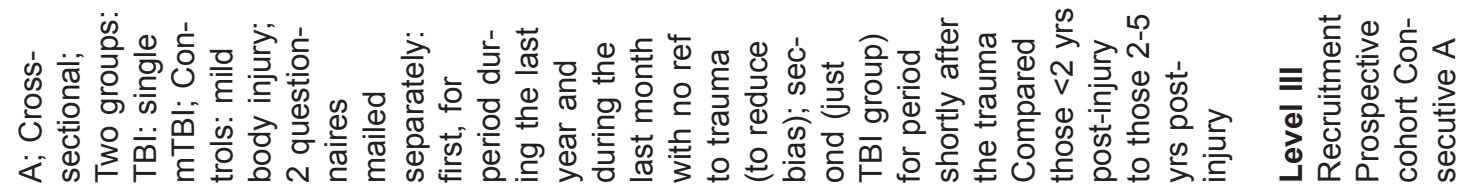
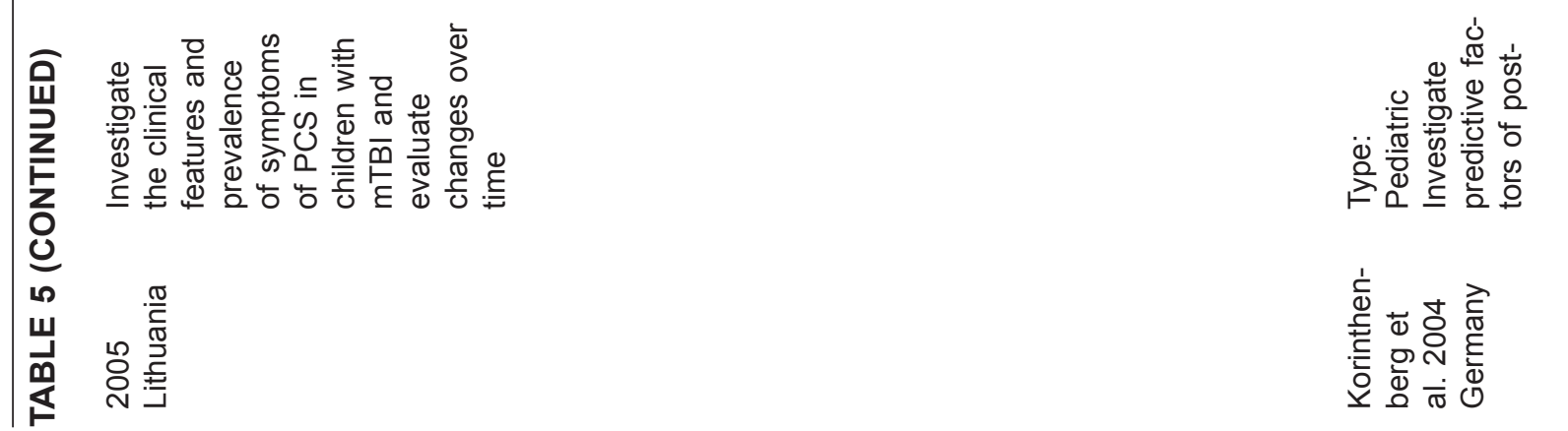


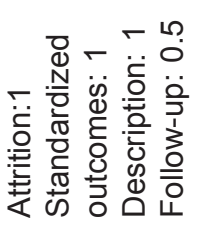

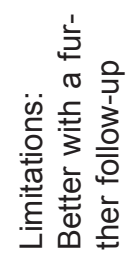

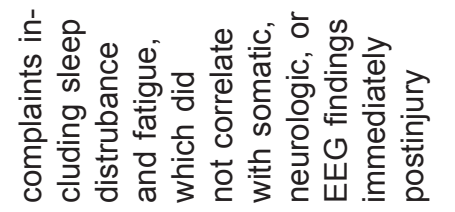

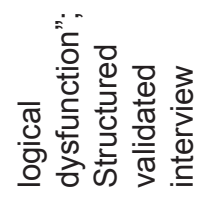

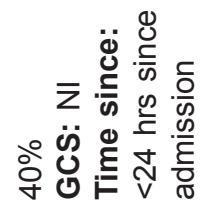

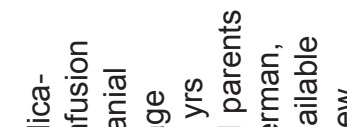

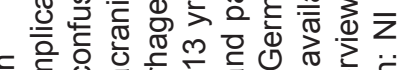

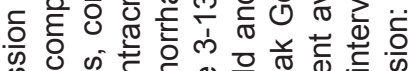

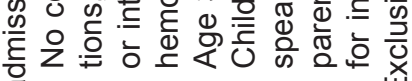

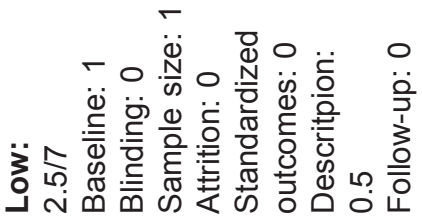

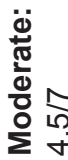

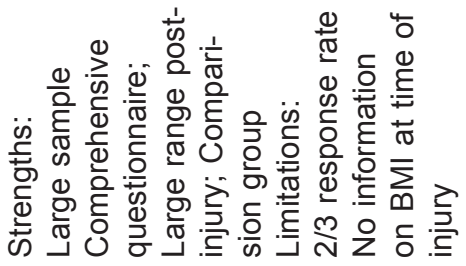

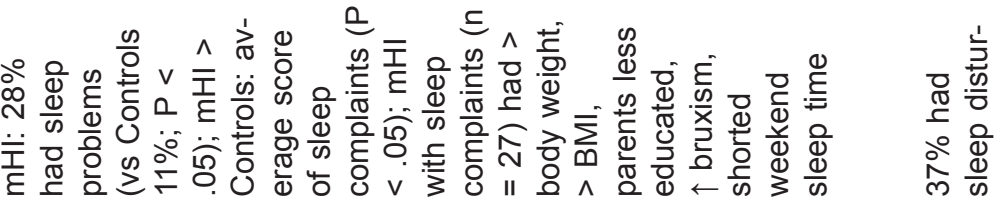

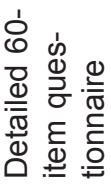
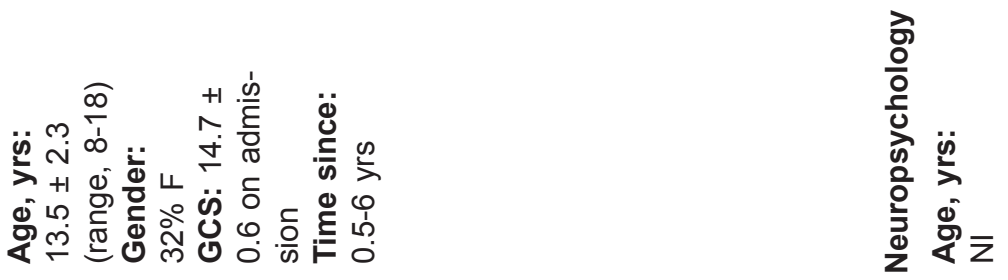

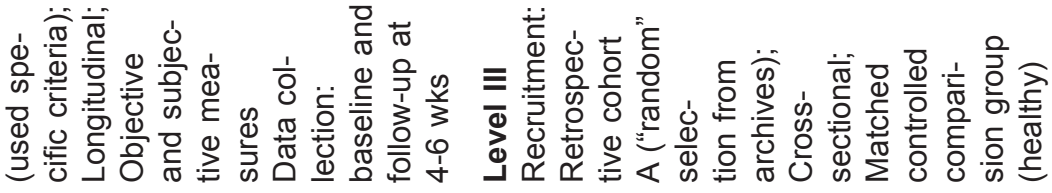

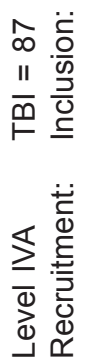
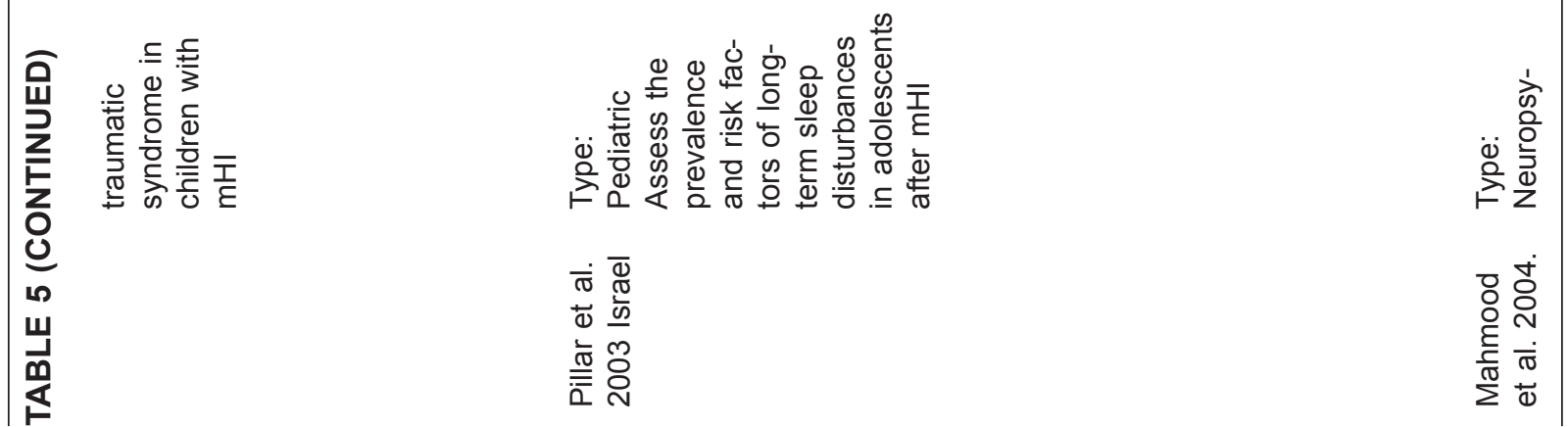


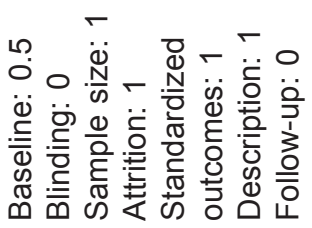

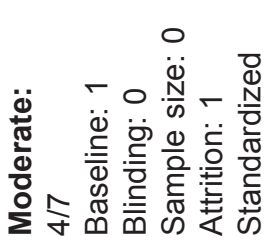

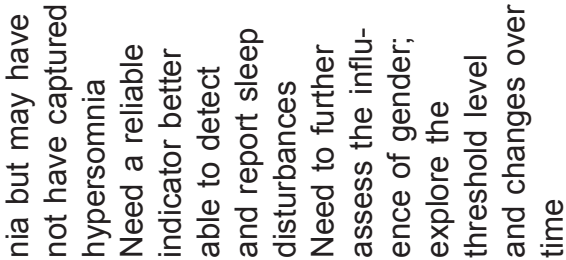

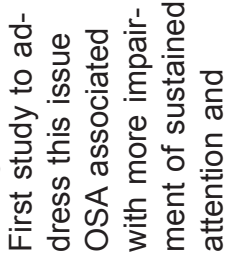

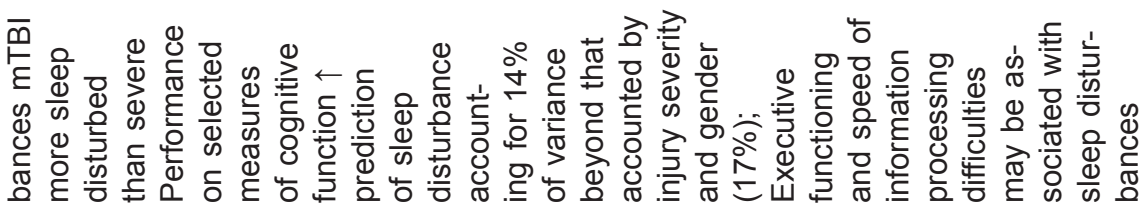

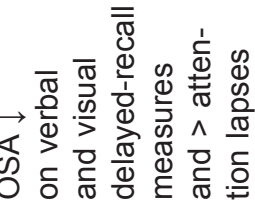

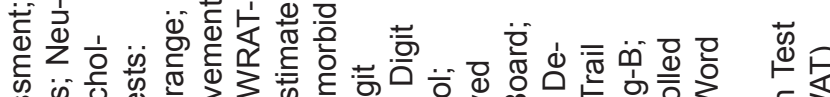

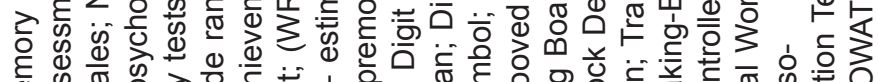

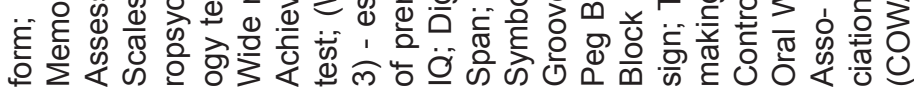

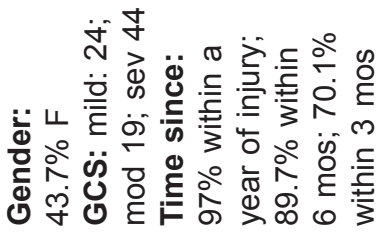

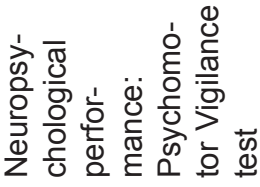

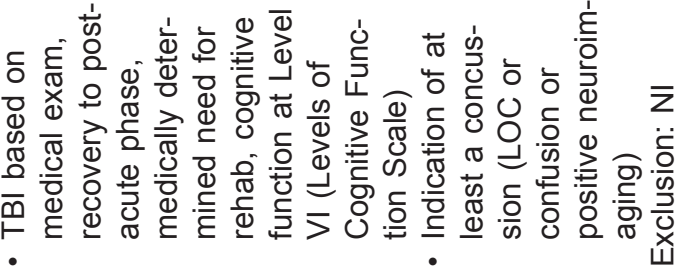

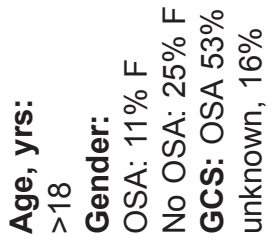

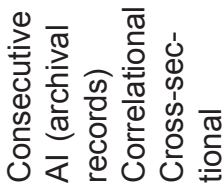

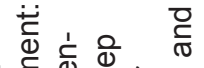

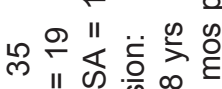
II 4 ○ी कू

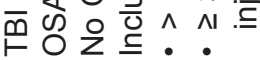

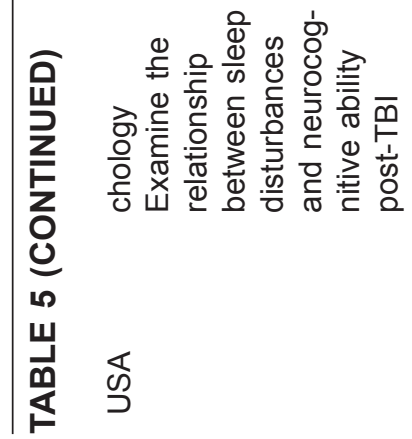

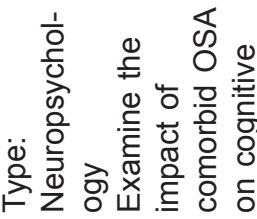

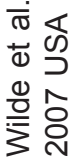




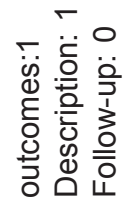

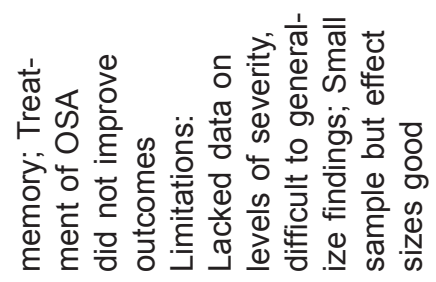

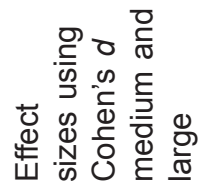

它产竞要

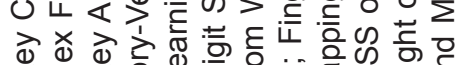

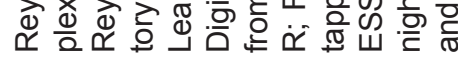

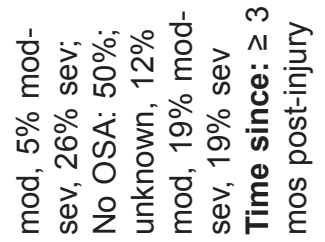

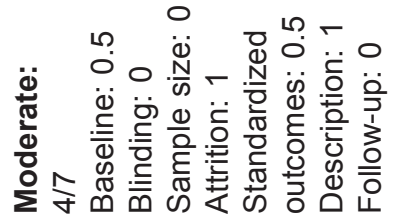
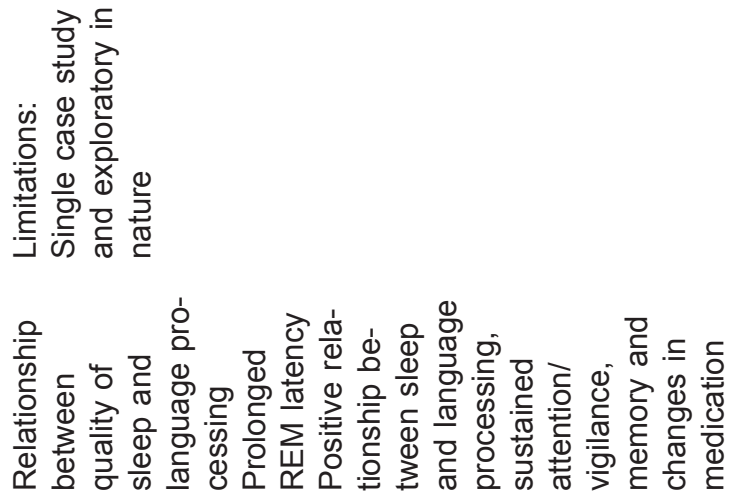

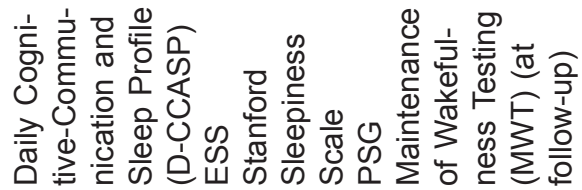

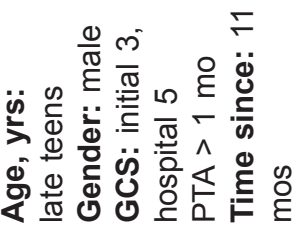

$\bar{z}$
$\ddot{\check{c}}$
$\frac{0}{9}$
$\frac{0}{0}$
य

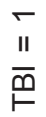

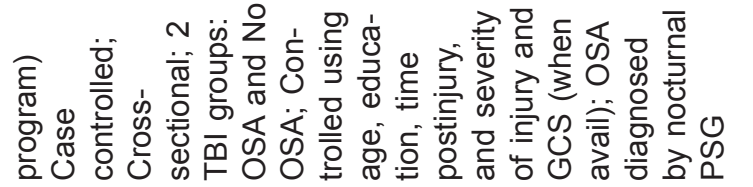

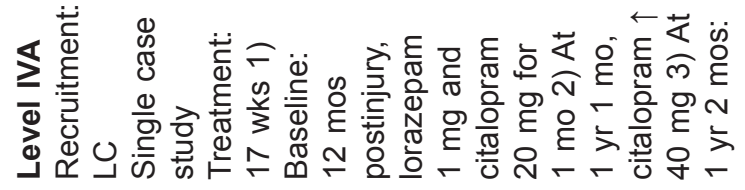

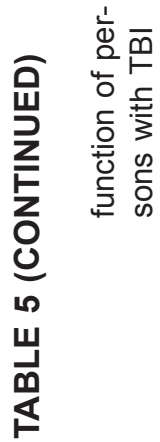

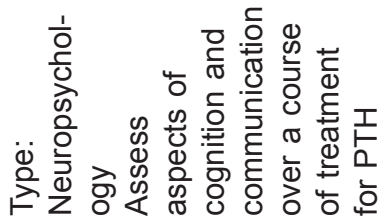

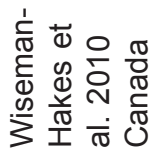



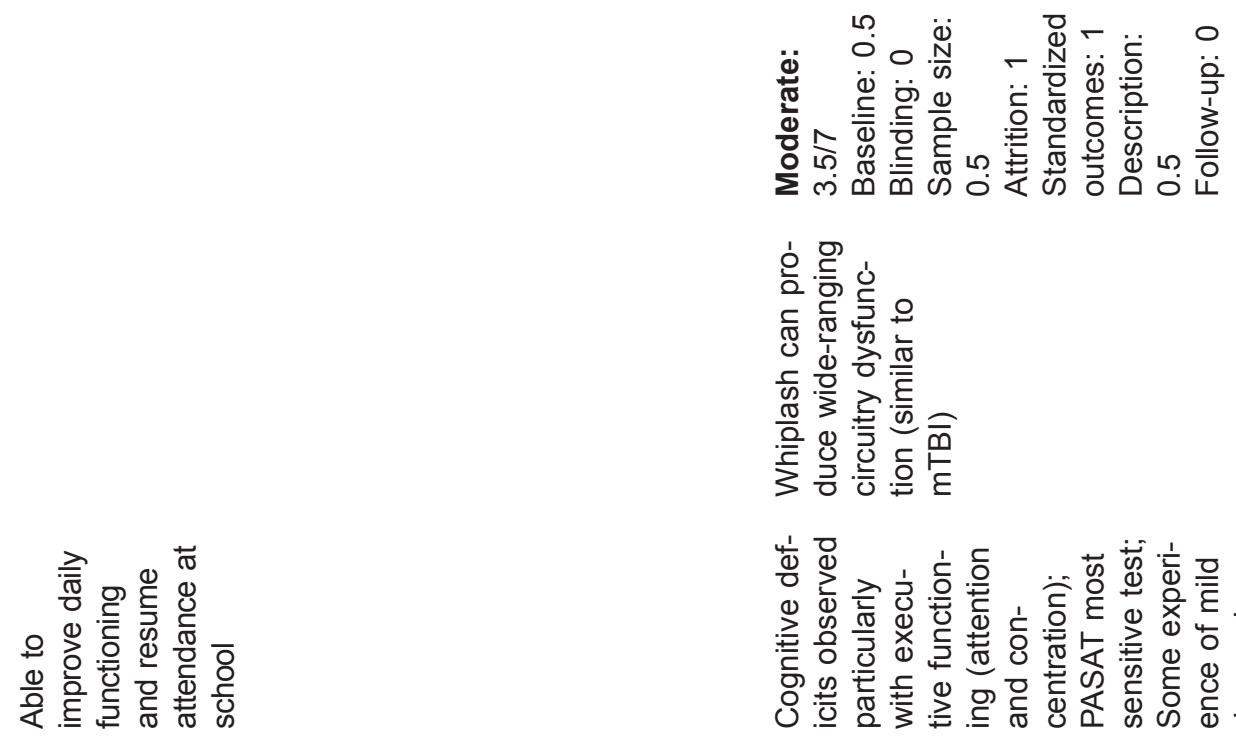

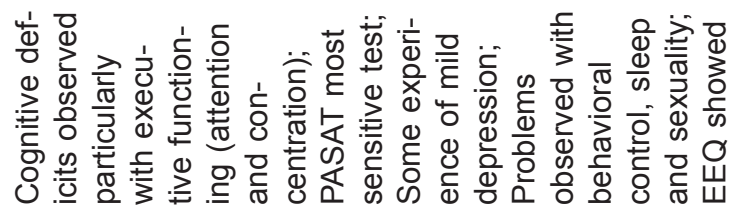
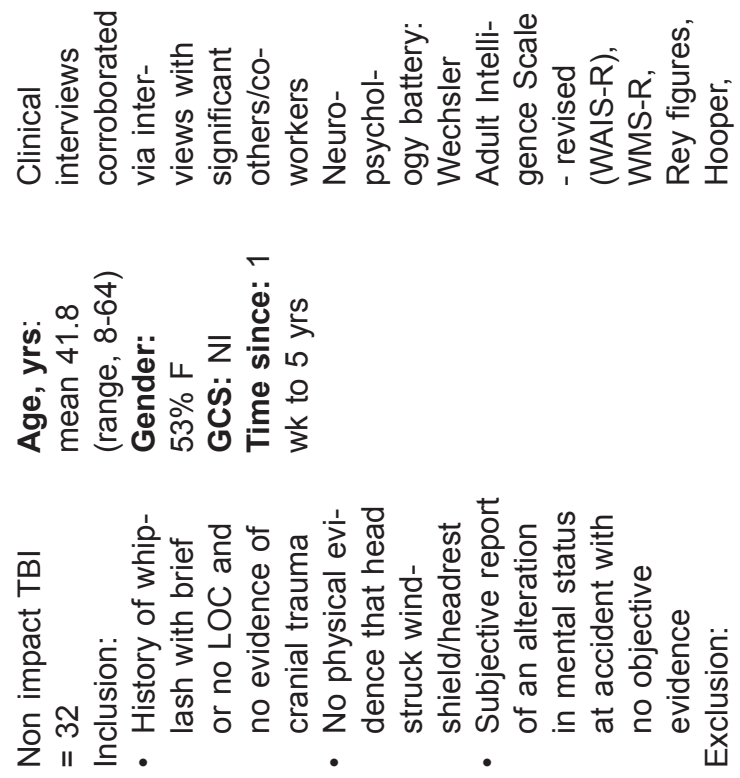

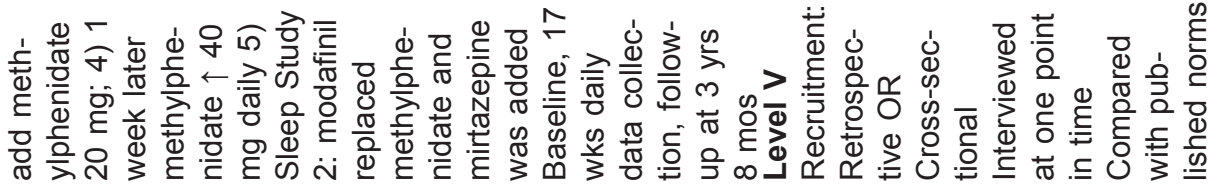

号

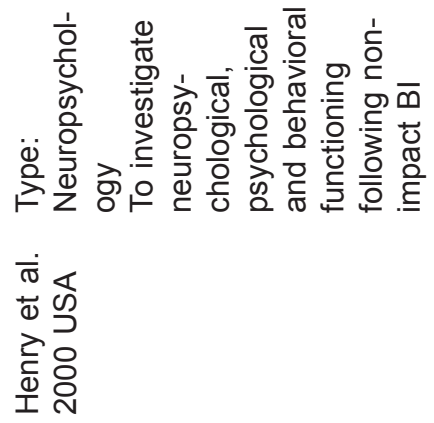




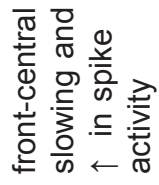

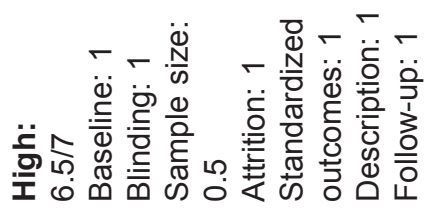

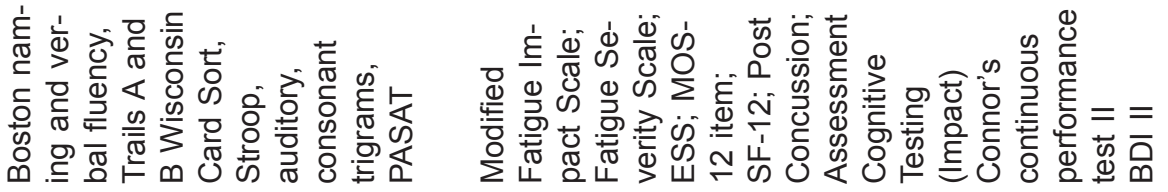

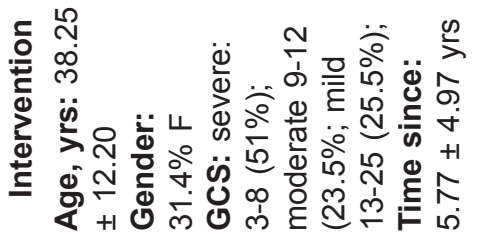

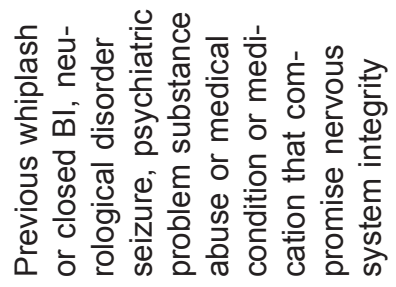

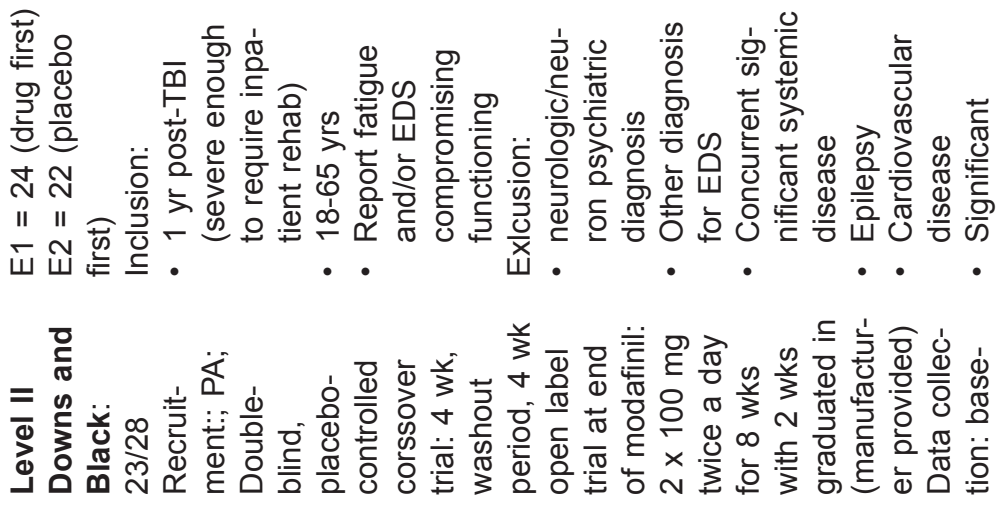

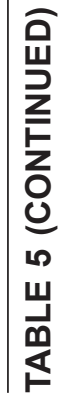

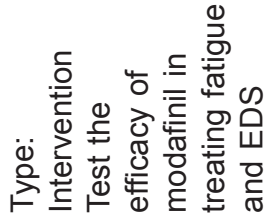

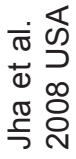



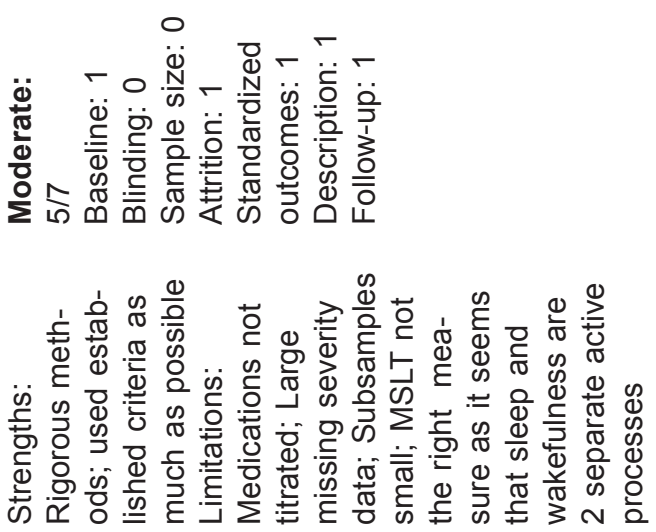

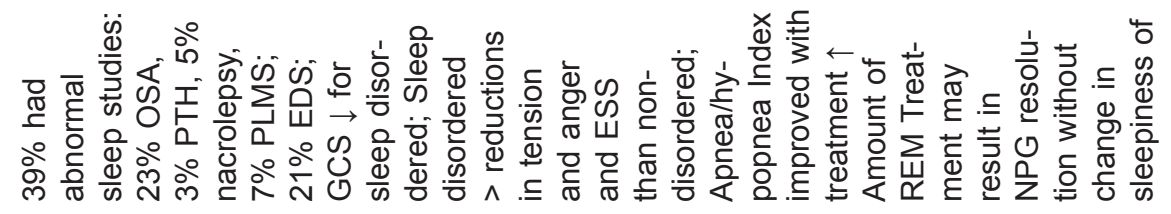

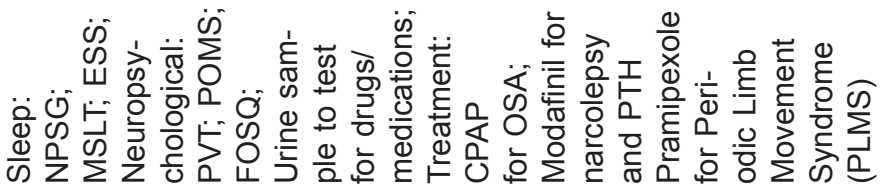

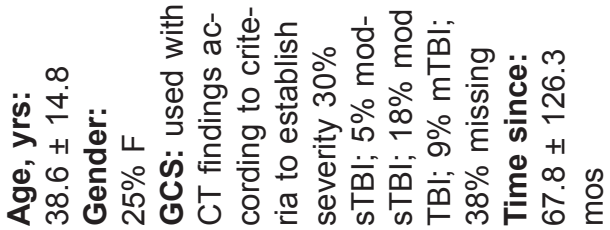

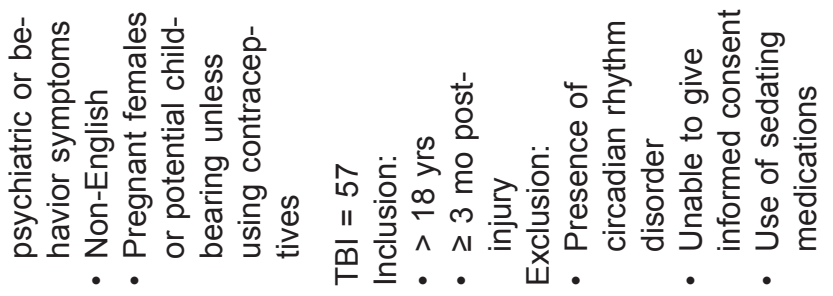

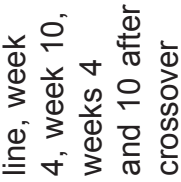

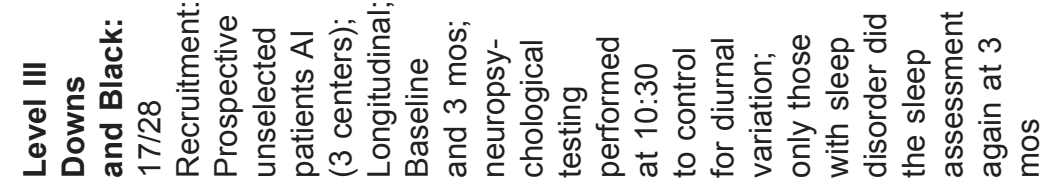

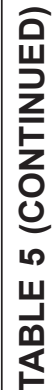

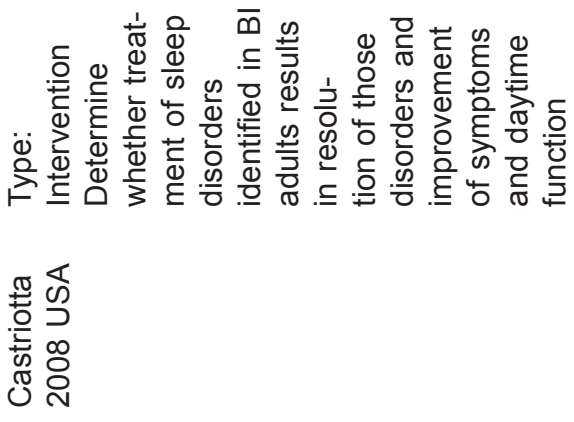




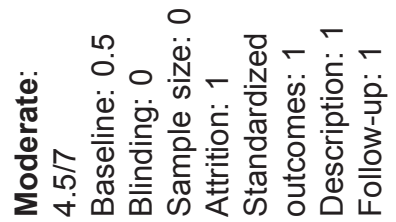

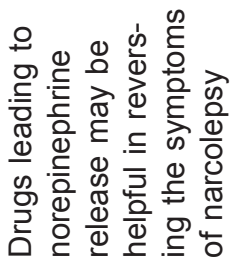

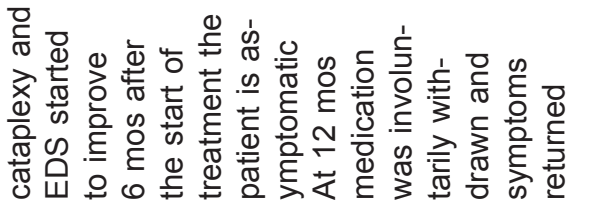

$\sum_{\substack{5 \\ 0 \\ 0 \\ 0 \\ 0}}^{5}$

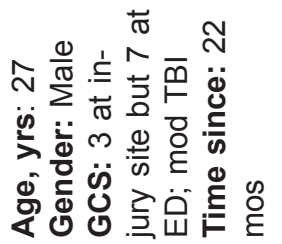

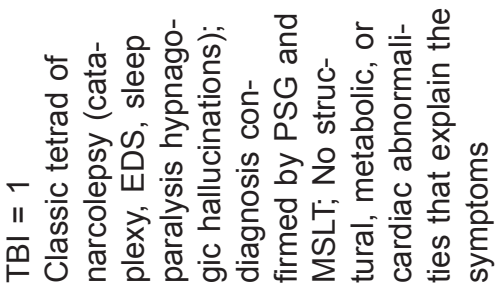

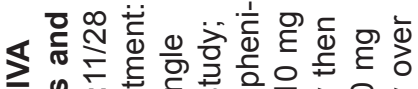

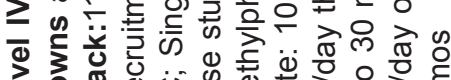

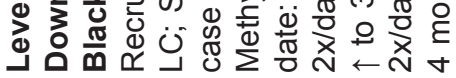

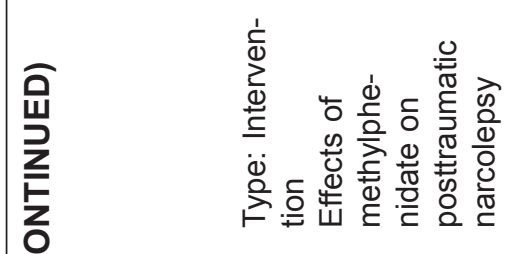

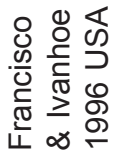

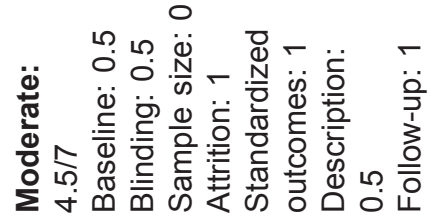

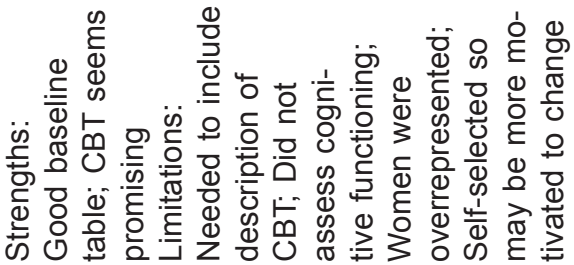

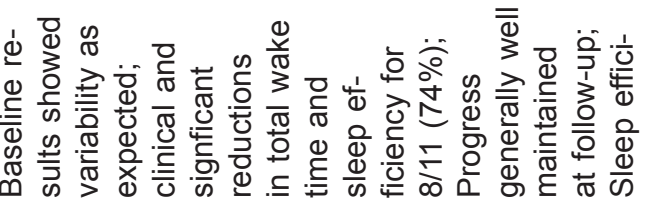

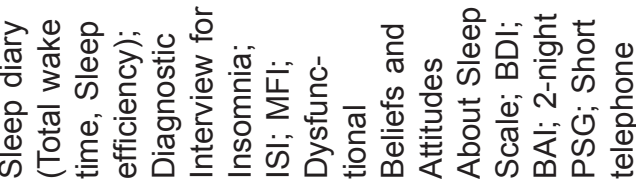

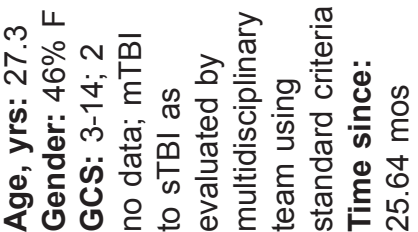

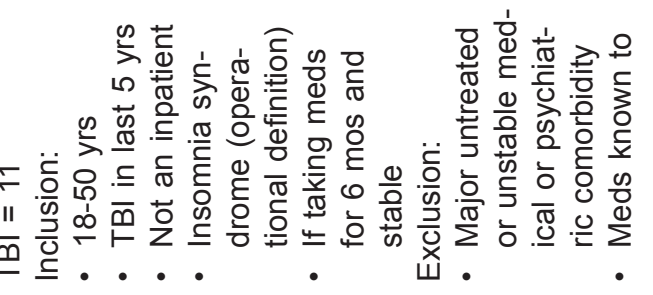

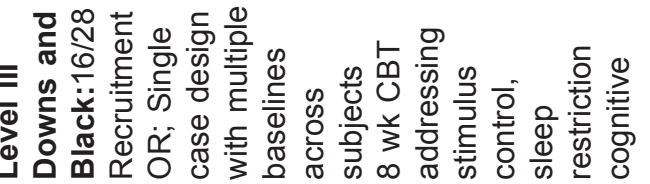

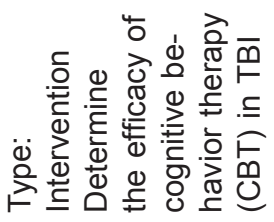

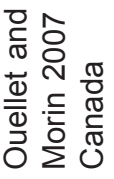



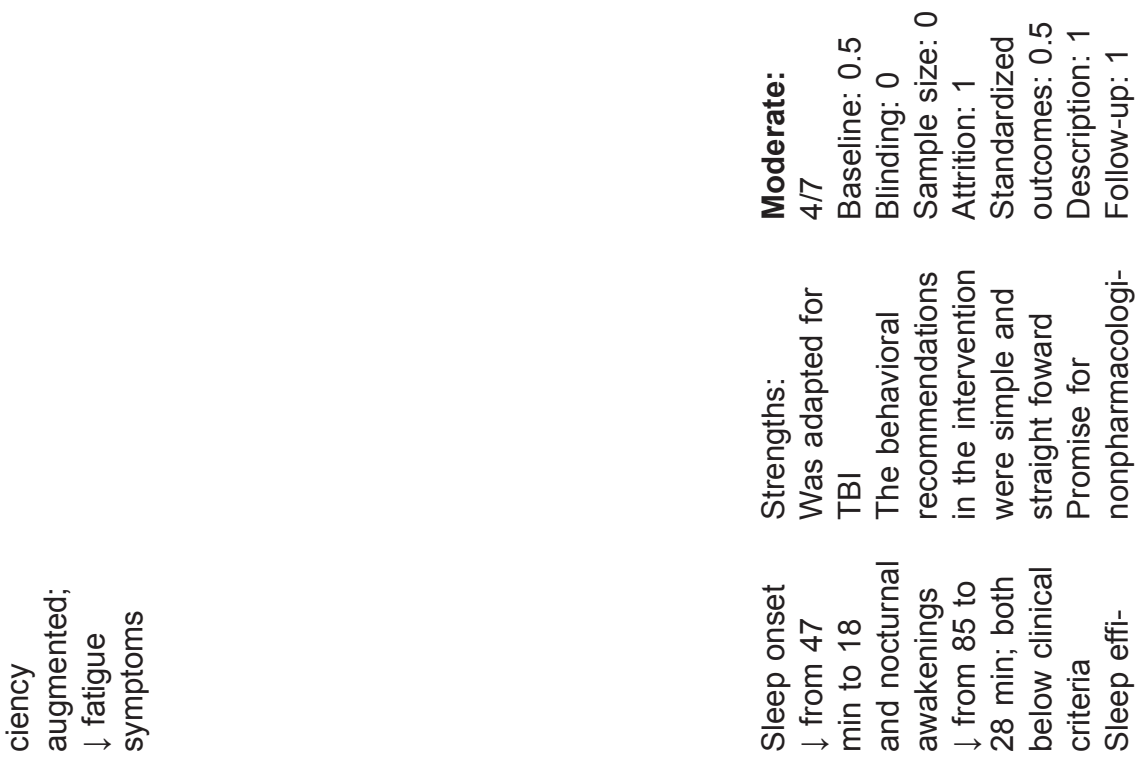

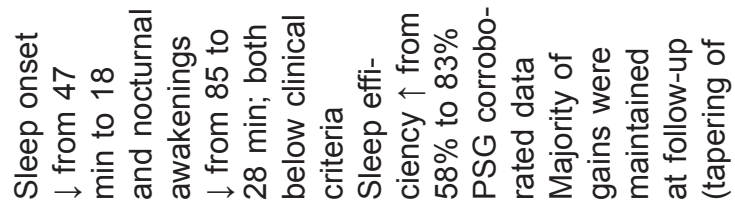

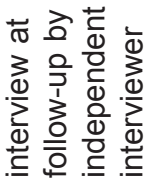

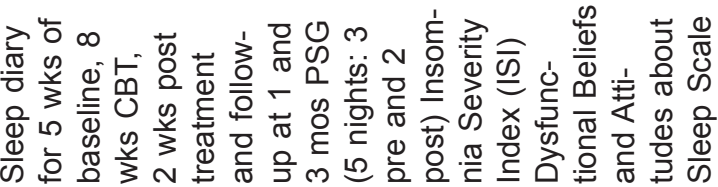
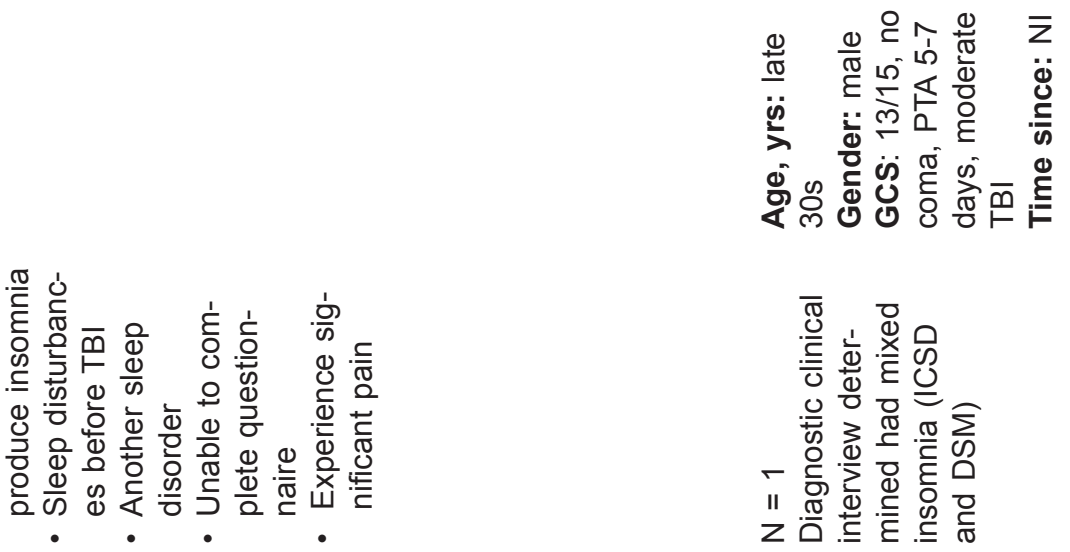

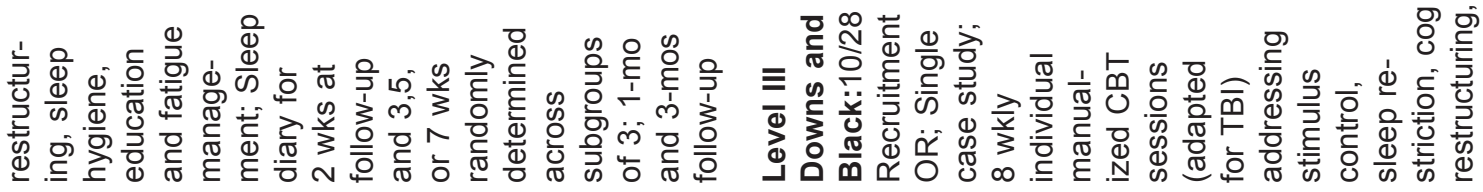

岂

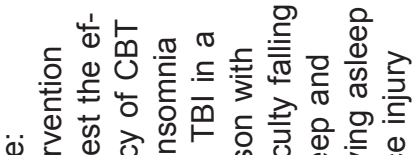

बं

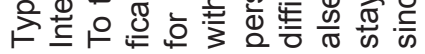

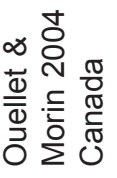




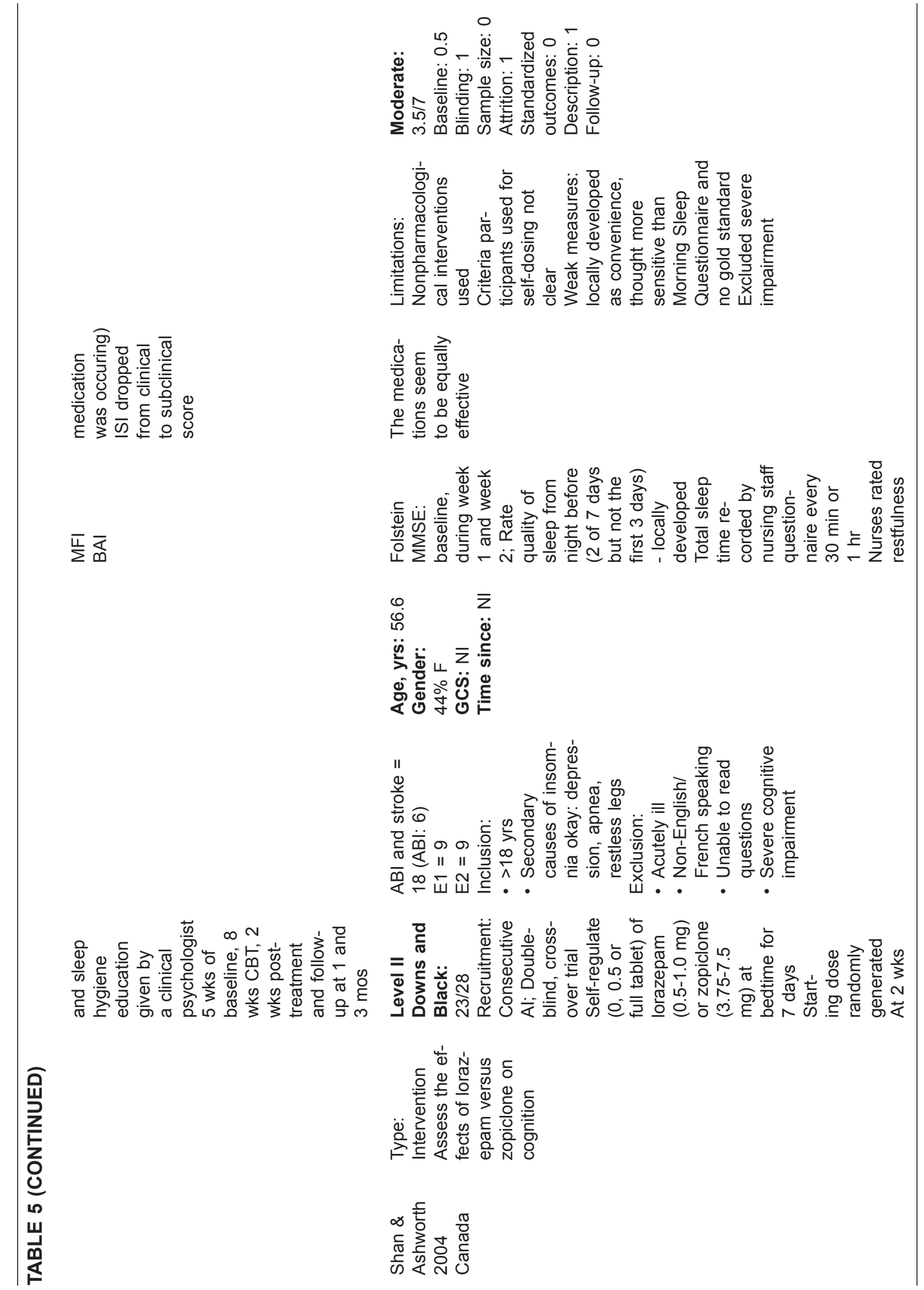




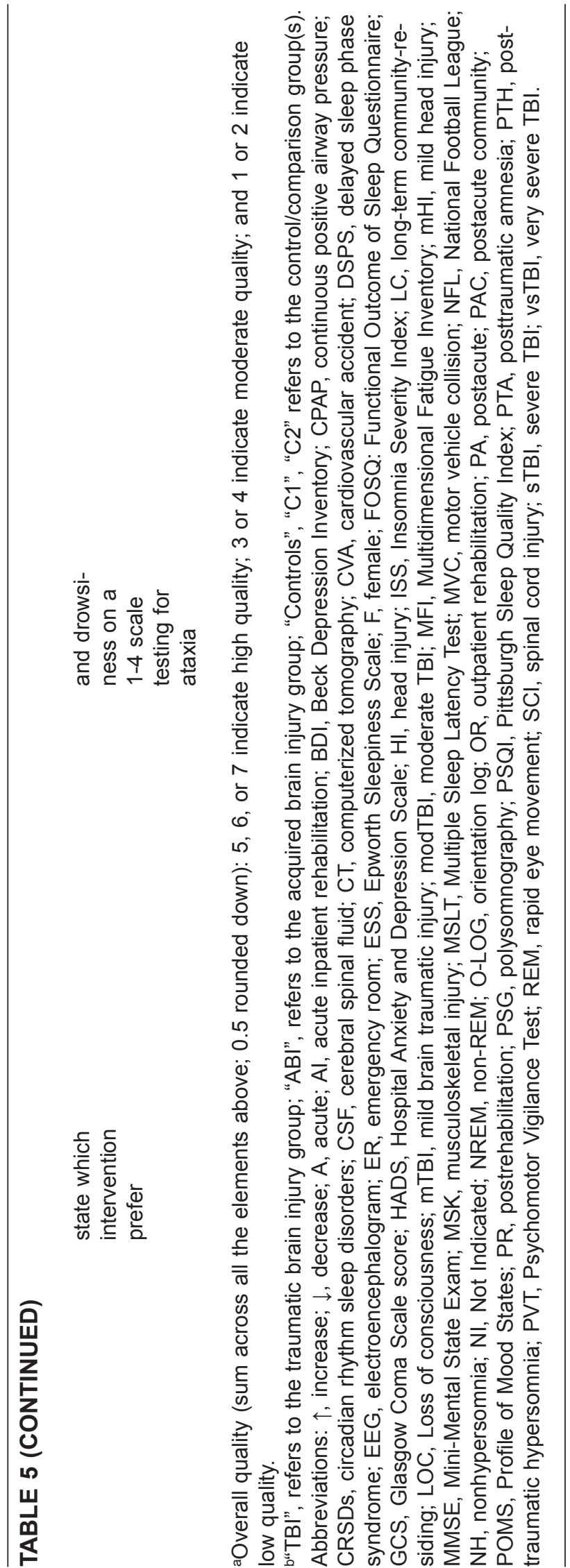


It has been consistently reported in the literature that individuals with TBI experience symptoms of insomnia and other sleep disturbances across the spectrum of recovery from the acute stage and beyond, across all levels of severity, with some patients continuing to report sleep disturbances for many years postinjury. ${ }^{4,6,29-37}$

Thus, in order to try and present the literature in a manner that might further elucidate our understanding of the developmental course of posttraumatic sleep disturbances over time, we attempted to discuss the studies from the perspectives of sleep in the acute stage, early recovery/ rehabilitation (to 1 year), and 1-3 years and beyond. However, consistent with the reports of Ouellet and colleagues ${ }^{6}$ and Orff and colleagues ${ }^{7}$ in their previous reviews, we experienced difficulties in doing so, particularly for those studies (the majority of which we identified) that were cross sectional, that is, evaluating sleep at only one time period.

Most studies clearly identified the average time postinjury of their participants and the range/ standard deviation, but the ranges were, in fact, quite wide. Among the studies that utilized a comparison group (these studies were methodologically stronger than studies that did not involve a comparison group), the ranges of time postinjury were the following (in chronological order from publication date): from 2.7 months to 5 years, average 2 years post; ${ }^{35} 2$ weeks to 53 months, average 3.8 months post; 327 to 41 months, average 20.96 months post $;{ }^{4} 20$ to 1194 days, average 230 days; ${ }^{29} 12$ months to 21 years, mean not given; ${ }^{36}$ 74 to 1194 days, average 516 days; ${ }^{26} 12.3$ to 43.3 months, average $27.8 \mathrm{mos}^{37}$ and up to 1 year post, specifics not given. ${ }^{27}$ Thus, among these studies, we identified an overall 21-year range in time postinjury of subjects. The results, however, were not stratified in any of the studies by time postinjury, thus making it extremely difficult to draw any definitive conclusions regarding the developmental time course of the sleep disorders.

Furthermore, sleep disorders may well be confounded over time by the subsequent development of depression, anxiety, reduced activity and weight gain, again making it difficult to draw specific conclusions about the etiology of these disorders. It is necessary to acknowledge and mitigate potential factors that may confound the results, whether these are factors that were preexisting to the brain injury or have developed secondary to the brain injury. However, from the perspective of recruitment at various times postinjury, there were studies that reported on sleep findings, including both prevalence and nature, at specific time points across different levels of severity. Thus, we have attempted to summarize the literature regarding the prevalence and nature of sleep disorders following TBI based on the source of patient recruitment: acute/postacute, rehabilitation, and community. Only those studies that delineated recruitment by time postinjury and level of severity, as well as stratified results according to the source of patients (e.g., acute care, rehabilitation settings) and level of severity, are included below. Thus, those studies that did not stratify their findings by level of severity are not included in our analysis. In addition, we only included studies that had substantial measures of sleep versus a single item documenting sleep problems.

\section{a. Patients Recruited From Acute and Post-acute Care}

We identified five studies that recruited participants from acute care settings. ${ }^{26,28,30,32,39}$

Acute mild. Chaput and colleagues ${ }^{38}$ conducted a retrospective chart review to determine the prevalence of sleep complaints in a sample of 443 patients diagnosed with mTBI. The authors used two time points: 10 days postinjury and again at 6 weeks postinjury. Of this sample, $13.3 \%$ reported sleep changes at 10 days postinjury, and this increased to $33.5 \%$ at 6 weeks postinjury with a smaller sample. Furthermore, the prevalence of sleep complaints at 6 weeks was 2.9 times more likely if such a symptom was present at 10 days $(P=.004)$ and was associated with concomitant headaches, depressive symptoms, and irritability. Given the large sample size and the fact that they recruited all of those patients within the diagnostic category rather than just those with sleep complaints, the sample bias is minimized and the results are more generalizeable to the whole mTBI population. The nature of the sleep complaints, however, was not delineated.

Acute and postacute moderate-severe. Makley and colleagues ${ }^{28}$ followed a sample of 14 patients 
with moderate-severe injuries recruited at 9 to 23 days postinjury from acute care, and reported that $78 \%$ had severely impaired mean sleep efficiency $(<63 \%)$, as measured by actigraphy. These patients were subdivided into two groups, those with ongoing posttraumatic amnesia (PTA) and those whose PTA had resolved. Of significance, they reported that sleep efficiency improved once patients were no longer in a state of PTA $(P=.032)$. Baumann and colleagues ${ }^{24}$ consecutively recruited 96 patients within the first 4 days after TBI; at 6 months following TBI, 65 patients were evaluated using a combination of polysomnography (PSG), Multiple Sleep Latency Test (MSLT), actigraphy and clinical interview. The authors determined that at 6 months postinjury, among patients across all levels of severity ( $60 \%$ moderate-severe), $72 \%$ had sleep problems, $28 \%$ had subjective daytime sleepiness, $25 \%$ had objectively measured daytime sleepiness, $17 \%$ reported fatigue, and $22 \%$ reported "sensu strictu" (an increased sleep need over 24 hours). An additional 5\% had insomnia. The authors further noted that in $43 \%$ of patients, the only possible cause of these problems was directly attributed to the TBI.

Rao and colleagues ${ }^{30}$ recruited a sample of 54 patients within 3 months of trauma across all levels of severity; however, all had experienced a loss of consciousness. Results of the Medical Outcome Scales for sleep indicated significantly increased sleep disturbance $(P=.018)$, decreased sleep adequacy $(P=.023)$, and increased daytime sleepiness $(P=.0002)$ in comparison to self-reports of preinjury sleep. Watson and colleagues ${ }^{39}$ looked specifically at the prevalence of hypersomnia in a consecutive sample of 346 patients with TBI recruited from admissions to a Level 1 trauma hospital, in comparison with trauma controls (without TBI) and trauma-free controls. Data on sleepiness were collected at 1 month postinjury, with followup at 1 year. The level of severity was determined by the time taken to follow commands according to the motor component of the Glasgow Coma Scale (GCS). Although 78\% of subjects took less than 24 hours to follow commands, they presented with serious enough symptoms in the emergency room that they were admitted to hospital and as such we have included them in our discussion of patients with moderate-severe TBI. At 1 month postinjury, $55 \%$ of all TBI subjects endorsed one or more sleepiness items on the Sickness Impact Profile, in comparison with $41 \%$ of trauma controls and $3 \%$ of trauma-free controls $(P<.001)$.

\section{b. Patients Recruited From Rehabilitation Populations}

Mild. We identified only four recent studies in which mild subjects from a rehabilitation outpatient population were recruited. Beetar and colleagues ${ }^{35}$ conducted a retrospective chart review to determine the prevalence of sleep complaints in a consecutive series of 202 patients with TBI referred for neuropsychological assessment. The sample included $127 \mathrm{mTBI}$ patients, 75 moderatesevere TBI patients, and 123 non-TBI controls. The authors found that, overall, $56.4 \%$ of the TBI patients had significantly more complaints of insomnia in comparison with controls $(30.9 \%$; $P<$ .001 ), and the mTBI group reported approximately $50 \%$ more insomnia complaints than those with moderate-severe injuries $(P<.001)$. This study was an important early contribution to the literature on sleep and TBI. Even though the authors did not use an operational definition of insomnia, their large sample size, use of a control group, and delineation of results by severity provided a strong foundation for future research. With regard to prevalence, Clinchot and colleagues ${ }^{40}$ also reported on a sample of patients recruited from consecutive admissions to rehabilitation and contacted at 1 year postinjury. The authors found that approximately $75 \%$ of subjects (GCS score of 13-15) subjectively reported sleep disturbance.

Schreiber and colleagues ${ }^{36}$ studied the nature of sleep disturbance, conducting a retrospective study of 26 patients with mTBI and sleep disturbance who were consecutively admitted to ambulatory rehabilitation. In comparison with healthy age- and sex-matched controls (who had routine sleep evaluations as part of a preemployment assessment procedure), the mTBI group had objective changes in sleep architecture, including increased light sleep (nREM) in $54.5 \%$ of subjects in comparison with controls $(46.6 \%)$, and significantly lower total sleep time $(P<.05)$. They also reported increased excessive daytime sleepiness objectively measured by MSLT in comparison with controls (higher number of falling asleep 
episodes $(P \leq .05)$ and shorter time to fall asleep $(P<.0005)$. Baumann and colleagues ${ }^{24}$ (also discussed in the section above on acute and postacute moderate-severe TBI) reported objectively measured sleep efficiency of $92 \%$ among their mild subjects; however, they identified 2 subjects (13\%) with sleep efficiency well below the age-adjusted $25 \%$ percentile values. It is important to consider that the findings of these three studies may not be generalized to the population of mTBI as a whole. Despite the fact that the subjects were classified as mild, they all required rehabilitation and/or were referred for neuropsychological evaluation, thus placing them in a more "involved or severe/ complicated" category of "mild" injury.

Moderate-severe. Although we identified seven studies that examined the prevalence and/or nature of sleep disturbances in samples recruited from a moderate-severe rehabilitation population, ${ }^{25,31,32,40-43}$ only the most recent (and higher quality) study stratified or reported results according to severity ${ }^{25}$ with the exception of the earlier work by Clinchot. ${ }^{40}$ Clinchot and colleagues $^{40}$ (recruitment described above) found that approximately $57 \%$ of patients with a GCS score of $8-12,24 \%$ of patients with a GCS score of 5-7, and $40 \%$ of patients with a GCS score of 3-4, reported subjective sleep complaints. Castriotta and colleagues ${ }^{25}$ conducted an objective evaluation of sleep in 87 patients who were at least 3 months postinjury and were recruited from the rehabilitation services at three academic medical centers. Abnormal sleep studies were found in $46 \%$ of subjects. With regard to the nature of the sleep disturbances, $20 \%$ had OSA, $11 \%$ had posttraumatic hypersomnia, 6\% had narcolepsy, and 7\% had PLM. Among all subjects, $22 \%$ had objective excessive daytime sleepiness as measured by the MSLT. Interestingly, they identified no differences in injury severity and/or time postinjury between sleepy and non-sleepy subjects with TBI.

Finally, we identified two studies that recruited patients from rehabilitation, and although they identified objectively measured sleep and wake disturbances among participants, neither study identified any significant differences in injury severity or time postinjury between sleepy and non-sleepy subjects. ${ }^{25,31}$ Castriotta and colleagues ${ }^{25}$ examined the prevalence of sleep disorders in a prospective sample of 87 patients with TBI who were recruited from the rehabilitation services at three academic medical centers. Abnormal sleep studies (PSGs) were found in $46 \%$ of participants, including $23 \%$ with OSA, $11 \%$ with posttraumatic hypersomnia, and 7\% with PLMs. Objective excessive daytime sleepiness was found in $25 \%$ of participants. Consistent with the findings of Verma, ${ }^{31}$ OSA was more common in obese participants (body mass index $[\mathrm{BMI}] \geq 30$ ). Furthermore, these investigators did not find any differences in severity of injury or time postinjury between sleepy and nonsleepy participants $(P<.05)$.

\section{c. Patients Recruited From the Community}

We identified eight articles in which samples of individuals with TBI who had returned to the community were recruited. 4,26,27,29,31,37,39,44

Mild. Williams and colleagues ${ }^{37}$ studied the extent and nature of sleep complaints in a sample of nine university students with previous mTBIs (range, 1.4-3.6 years postinjury) in comparison with age- and gender-matched controls. They identified 4\% less efficient sleep, shorter REM onset latencies, and longer sleep onset latencies in comparison with controls, objectively measured by PSG. Although this study is quite thorough and rigorous in design, the small sample size and "sample of convenience" (i.e., student volunteers), weakens the generalizability of the findings. Schreiber and colleagues ${ }^{36}$ characterized the nature of sleep disturbances in a sample of 26 chronic mTBI patients with sleep complaints who were at least 1 year postinjury. They reported changes in sleep architecture (as measured by PSG), including increased light non-REM sleep, in $54.5 \%$ of patients $( \pm 13.4 \%)$ in comparison with controls $(46.6 \% \pm 10.4 \%)$.

Moderate-severe. Parcell and colleagues ${ }^{29}$ reported on a sample of 63 participants with TBI consecutively recruited after discharge from rehabilitation, in comparison with 63 age- and sex-matched controls. Eighty percent of patients reported subjective changes in sleep relative to controls $(23 \%)$, including more nighttime awakenings, and longer sleep-onset latency. In another study, ${ }^{26}$ Parcell and colleagues recruited 10 community-based participants with TBI and 10 age- and sex-matched controls. The authors 
reported objectively measured increases in slow-wave sleep, reductions in REM sleep, and reduced sleep efficiency in comparison with controls, as well as subjective reports of poorer sleep quality.

Ouellet and colleagues ${ }^{4}$ recruited a sample of 452 participants from mailings distributed to 1500 people with TBIs identified through the archives of the rehabilitation center, as well as TBI associations and support groups. Their sample included $59.9 \%$ with severe injuries, $23.3 \%$ with moderate injuries, $13.7 \%$ with mild injuries, and $3.1 \%$ with "minor" TBIs. Participants completed a detailed questionnaire that was then mailed back to the authors, who identified that $50.2 \%$ of their sample reported insomnia symptoms and $29.4 \%$ fulfilled the diagnostic criteria for an insomnia syndrome. This study continues to have the largest sample size of all of the literature to date pertaining to the prevalence of insomnia following TBI. In addition, it is one of the few that actually delineated between those who presented with symptoms of insomnia and those who met the diagnostic criteria for insomnia, thus providing a more reliable and valid estimate of the true prevalence. It is not possible, however, to completely eliminate sample bias without having information regarding the 1000 others who chose not to participate.

In another study, Ouellet and Morin ${ }^{44}$ examined the nature of insomnia in the context of TBI by recruiting a sample of 14 adults with TBI who presented with an insomnia syndrome (nine of whom were moderate-severe), and 14 healthy, age- and sex-matched controls. It was not clear from the article how or where the participants were recruited. The authors reported that $71 \%$ of subjects had objective findings of insomnia, including more awakenings and shorter REM onset latencies. Furthermore, in comparison with controls, those with TBI had significantly more awakenings lasting longer than 5 minutes $(P=$ $.059)$, as well as significantly shorter REM latency (for those with TBI taking no medications) $(P=$ $.050)$. In the 1-year follow-up to their study of the prevalence of hypersomnia in the acute stage following TBI, Watson and colleagues ${ }^{39}$ found that $27 \%$ of participants continued to endorse subjective symptoms of sleepiness, in comparison with $23 \%$ of trauma controls and $1 \%$ of nontrauma controls $(P<.001)$. However sleepiness did im- prove in $84-100 \%$ of participants with TBI, with the smallest improvement being $84 \%$ in the mildest group ( $\leq 24$ hours to respond to commands). Verma and colleagues ${ }^{31}$ objectively examined the nature and spectrum of sleep disorders in chronic TBI by recruiting a sample of 60 patients who presented with postinjury sleep complaints. It is not clear where or how the participants were recruited. Sixty percent of patients in the sample had moderate-severe injuries. Hypersomnia was the presenting complaint in $50 \%$ of all participants, which the authors reported was "mostly due to" underlying sleep apnea, narcolepsy, and PLMs. Of interest, $45 \%$ of the sample exceeded a BMI of 30, which is considered to be obese. Insomnia was the presenting complaint in $25 \%$ of participants, and half of those had difficulties with sleep onset.

Sport-related concussion. New to the literature is a 2009 study that evaluates sleep following sport-related concussions. Gosselin and colleagues ${ }^{27}$ examined both subjective and objective sleep quality in 10 subjects/athletes with a sportrelated concussion, and compared their findings to those of 11 nonconcussed control athletes from noncontact sports. The concussed group had a history of $4.6( \pm 2.1)$ concussions on average, sustaining at least one in the previous year. Objective measures of sleep included two consecutive nights of PSG, and electroencephalography (EEG) spectral power; subjective measures of sleep included the Pittsburgh Sleep Quality Index (PSQI), Epworth Sleepiness Scale (ESS), and Post Concussion Symptom Scale, as well as a subjective questionnaire that was developed specifically for the study. The authors reported that although the concussed athletes identified more symptoms and worse sleep quality than controls, this was not corroborated by any polysomnographic variables, or REM and nREM sleep quantitative EEG variables. However, concussed athletes showed considerably more relative delta activity, and reduced alpha activity and relative alpha power, during wakefulness than controls. Based on these findings, the authors concluded that sport-related concussions are thus associated with wakefulness problems, rather than sleep disturbances per se. Although this study has a small sample size and as such the Gosselin and colleagues' findings are preliminary, their strong methodological design is 
such that this is an important first step in examining this population. Their findings are relevant and timely, given that sport-related concussions occur at a predictable rate in "high-risk" sports (i.e., football, hockey, etc.), ranging from $7-12 \%$ of the participant population in any given year, and that the sequelae of sport-related concussion is currently receiving much attention. ${ }^{45-47}$

\section{d. Prevalence}

As the literature has continued to evolve, we have attempted (as have others before us) to identify ranges of the prevalence of sleep disturbances identified among the studies based on time postinjury of recruitment. The results of these studies confirm that disorders of sleep and wakefulness are prevalent across all stages of recovery from TBI, from the early acute stage until many years postinjury, and across all levels of severity. However, defining conclusive "ranges" continues to be problematic due to the heterogeneity within and between samples, inconsistencies in the measures used, different study objectives, and variations in reporting. Some studies utilized percentages for reporting findings; alternatively, others reported on regression analysis of sleep, along with various associated phenomenon (i.e., depression, anxiety); identified changes in sleep architecture; looked specifically at insomnia as a global phenomenon; reported on the symptoms of insomnia; or, looked at other types of sleep disorders such as hypersomnia in and of itself, or secondary to apnea. As such, a valid synthesis of prevalence rates among studies remains elusive, and larger epidemiological studies across levels of severity and varying time points are warranted.

\section{e. Methodological Limitations}

Although the studies continue to become more scientifically rigorous as the literature evolves, the results to date are representative of methodological limitations in study design, as most of the literature is based on a clinical subset of TBI survivors who: a) present with sleep complaints, therefore introducing bias into the sample; and $b$ ) have received rehabilitation and therefore represent a subset of this patient population with likely more severe injuries, even those who are classified as "mild". Thus it is likely that the data are skewed and are not fully representative of the TBI population as a whole. However, Parcell and colleagues ${ }^{26}$ specifically identified that their sample of 10 included "the first ten willing participants, with no preference for patients with sleep complaints," and Fichtenberg and colleagues ${ }^{32}$ recruited a prospective sample of 50 consecutive admissions to an outpatient rehabilitation program. The advantage of recruiting consecutively upon admission to the acute setting or from the emergency room is that all patients diagnosed with TBI are included in the study sample and followed regardless of whether they require postacute care. By following patients from the acute stage the issue of time since injury becomes negated, as all of the sample will be entered into the study at time of injury. As soon as recruitment takes place after the acute stage, researchers are already introducing forms of selection bias into the sample that should be addressed when discussing the generalizability of the findings. Given that such a small percentage of the studies were able to recruit consecutively from the acute setting the epidemiological understanding of this phenomenon is going to be fraught with issues of generalizability and difficulty in making clear conclusions.

An ideal study would follow a large sample consecutive series of patients from acute care for at least three years to understand how sleep problems manifest themselves, or not, and how these change over time, considering natural recovery by levels of severity.

Confounds. Some of the researchers made sure to exclude concurrent potential confounders, whereas others collected data on potential confounders (usage of caffeine, alcohol, medications, or drugs, BMI, and emotional state). However, with the exception of emotional state, which is also identified as a concurrent risk factor, the results were not stratified according to any of these confounds, nor were they addressed in discussion. Thus, we are only able to comment that these confounders exist, but are not able to make any definitive comments regarding their influence.

Risk factors. Ten studies identified risk factors specifically associated with insomnia following TBI, and two studies identified risk factors for 
other types of posttraumatic sleep and wake disorders. Beetar and colleagues ${ }^{35}$ identified pain and mTBI as being risk factors for insomnia complaints in a case-controlled sample of 200 TBI patients of mixed severity. Among a sample of 91 consecutive patients admitted to an outpatient rehabilitation program, Fichtenberg and colleagues $^{41}$ identified three risk factors associated with insomnia including milder injuries, the presence of pain, and depression. Of the three factors, depression had the strongest association with insomnia. Consistent with Fichtenberg's findings, Ouellet and colleagues ${ }^{4}$ reported that among a sample of 460 survivors recruited from the community (averaging 7.85 years postinjury, $83.2 \%$ of whom with moderate-severe injuries), risk factors for insomnia included milder injuries, depression, and pain. They also identified high levels of fatigue as a further risk factor.

Among a sample of 60 patients with varying levels of severity, ranging from 3 months to 2 years postinjury, Verma and colleagues ${ }^{31}$ noted that those with complaints of insomnia had elevated Beck Depression Inventory (BDI) scores and Hamilton Anxiety scores, thus further confirming that depression and anxiety are risk factors for insomnia following TBI across all levels of severity. They also identified $45 \%$ of their sample as having a significantly high BMI. Chaput and colleagues ${ }^{38}$ identified headaches, depressive symptoms, and irritability as co-occurring with sleep complaints in a sample of 443 patients with mTBI, at both 10 days and 6 weeks. Rao and colleagues ${ }^{30}$ also identified depression and anxiety as coexisting risk factors for symptoms of insomnia. Clinchot and colleagues ${ }^{40}$ was the only study to identify gender (female) as a risk factor for symptoms of insomnia $(P=.033)$, in addition to older age $(P=.035)$, milder injuries, and a GCS score greater than 7 $(P=.034)$. Baumann and colleagues ${ }^{24}$ identified that severe TBI (sTBI) was associated with the development of posttraumatic hypersomnia $(P=$ .02 ), but not with the presence, characteristics, and severity of other posttraumatic sleep disorders. The association between more severe TBI and the development of hypersomnia was also confirmed by Watson and colleagues. ${ }^{39}$ Castriotta and colleagues $^{25}$ identified that among the $46 \%$ of their sample with abnormal sleep studies (with five different types of sleep and/or wake disorders), the sample participants were significantly older than the nonsleep-disordered subjects $(P<.01)$ and had a significantly higher BMI $(P<.05)$.

Sample size. TBI is very heterogeneous and as a result it is complicated to conduct research that is generalizable. Ideally, studies would be able to recruit a large number of patients so that stratification on a number of variables would be possible: age at injury, gender, etiology of injury, severity of injury, and time since injury. Only $39 \%$ $(9 / 23)$ of the studies reviewed used sample sizes of greater than 75 . Thirty-five percent $(8 / 23)$ of the studies involved studies with sample sizes of less than 30 and thus were compromised in their statistical analysis and their ability to generalize to the different subgroups of brain injury.

Definition of insomnia. Another methodological limitation, also identified by Ouellet and Morin, ${ }^{44}$ is the lack of an operational definition of insomnia. Among the five comparison group studies that evaluated subjective reports of insomnia, sleep, and hypersomnia postinjury, ${ }^{29,32,35,39,44}$ only two utilized an operational definition of insomnia, the International Classification of Sleep Disorders and the DSM-IV. ${ }^{32,44}$ Related to this is the lack of consistency in or development of an operational framework for assessing severity of brain injury. Many studies use the GCS score to assess severity but the GCS score is not always available and other factors are included: length of PTA, period of loss of consciousness, cerebral computed tomography (CT) findings, and neuropsychological findings. One study ${ }^{39}$ determined injury severity based on time taken to follow commands. Baumann and colleagues, ${ }^{24}$ one of the two methodologically strongest studies reviewed, specifically cited using GCS scores and CT findings based on the Marshall ${ }^{48}$ criteria $(\mathrm{I}=$ no visible intracranial pathology, II-IV = midline shift, and $\mathrm{V}=$ mass lesion). It is necessary that there be consistency as to how to combine all of these factors together so that severity distinctions can be enabled when only some of the above features are present.

Measures. As per the comments of Orff and colleagues, ${ }^{7}$ there is also a significant discrepancy among the measures utilized. Of the 23 studies we evaluated under the epidemiology category, investigators used 19 different methods to evaluate sleep, of which four are objective (PSG, actigraphy, MSLT, Multiple Wake Test [MWT]). There is no 
gold standard of standardized instruments that is accepted. Although locally developed measures and those that are clinically developed may be very useful, it is impossible to compare from one study to the next when different types of measures are being used to assess the outcomes. The issue is that sleep itself is very difficult to assess, as it is composed of many components. It would seem that among the validated instruments, the most commonly used and accepted are the Diagnostic Interview for Insomnia (DII), Insomnia Severity Index (ISI), PSQI, and the ESS. It has been proposed that the MWT may be more suitable for this population than the MSLT, as daytime sleepiness seems to be a hallmark of this condition. One study ${ }^{30}$ utilized the 12-item Medical Outcome Scale for Sleep, ${ }^{49}$ which has been found to have good psychometric properties and has been found to be useful for assessing sleep problems in adults. Each of these instruments contributes a unique component to the assessment of insomnia and sleep difficulties and as such should be used in combination, rather than in isolation.

The subjective or self-report measures ranged from the early work of Beetar, which relied on a chart review, and concluded that "a sleep problem was judged as present if it was mentioned in the chart as reported by the patient," ${ }^{35(\text { p. } 1299)}$ to the more robust PSQI used by Fichtenberg ${ }^{32}$ (and validated for use with TBI patients), the battery used by Ouellet and colleagues ${ }^{44}$ that included the DII, ISI, Multidimensional Fatigue Inventory, in addition to other measures and nocturnal PSG, sleep diaries, and ESS used by Parcell and colleagues. $^{26,29}$ The most commonly used measures included the PSQI, PSG, and some form of a sleep diary. It is positive to note that as the literature evolves, overall, the use of measures to evaluate sleep and wake disorders have become increasingly comprehensive, sensitive, and "robust" over time. This in and of itself, however, further emphasizes the weakness of recent studies that rely on limited and/or weak measures, such as the study by Watson and colleagues. ${ }^{39}$ Although we recognize that this study was part of a larger study and had the strength of a follow-up in its design, the sole reliance on data obtained via four questions about sleep from the Sickness Impact Profile weakens the validity/generalizability of the overall findings.

\section{TABLE 6}

\section{Practice Points}

1. Sleep and wake disorders are present across all levels of severity at all stages across the continuum of recovery following TBI.

2. Sleep and wakefulness should be routinely and systematically assessed for the duration of medical and neuropsychological follow-up after TBI.

3. Risk factors for insomnia include $\mathrm{mTBI}$, pain, anxiety, depression, fatigue, and older age.

4. Risk factors for hypersomnia include more severe TBI.

5. Increased BMI $(>30)$ is a risk factor for OSA.

6. The PSQI, followed by the ISI, are appropriate measures for initial screening and corroboration of the impact of the problem.

\section{Research Agenda}

1. A large scale multi-center collaborative trial involving all those with TBI (i.e., not limited to those who present with sleep problems) recruited in the acute stage, followed longitudinally into the community and evaluated at regular time points is warranted.

2. Functional measures should be used in addition to formal measures of cognition. 


\section{Pathophysiology}

We identified three papers, all of moderate quality, which look at varying aspects of pathophysiology of sleep/wake disturbances following TBI, including two Level III studies ${ }^{50,51}$ and one Level IVA study. ${ }^{52}$ In 2005, Baumann and colleagues ${ }^{50}$ sought to test the hypothesis that deficiencies in hypocretin-orexin-1 (Orexin A) neurotransmission in acute TBI may play a role in the emergence of subsequent sleep/wake disorders. Orexin A is an excitatory hypothalamic neuropeptide involved in the regulation of the sleep/wake cycle, and its levels are typically reduced in persons with narcolepsy. Hypocretin-1 levels were measured in 44 patients by radioimmunoassay at $1-4$ days following TBI (males, $\mathrm{n}=32 ; 31$ patients with sTBI, 8 with moderate TBI [modTBI], and 5 with $\mathrm{mTBI}$ ), and compared with hypocretin-1 levels in healthy controls $(n=20)$. Their results indicated that in comparison with controls, hypocretin-1 levels were abnormally lower in $95 \%$ of patients with moderate-severe TBI, and in $95 \%$ of patients with posttraumatic brain CT findings. The authors postulated that hypocretin-1 deficiency following TBI may be reflective of hypothalamic damage (specifically to the posterolateral hypothalamus) and may be linked with the frequent development of posttraumatic sleep wake disorders. These findings are an important contribution to the determination of risk factors.

Ayalon and colleagues ${ }^{51}$ conducted a study to diagnose and describe the physical and behavioral characteristics of circadian rhythm sleep disorders (CRSDs) in patients with mTBI. Forty-two patients with mTBI and complaints of insomnia were screened, and those who were suspected of having CRSDs underwent further diagnostic evaluation. In total, 15 of the 42 patients ( $36 \%$ ) were formally diagnosed with CRSDs, eight patients displayed a delayed sleep phase syndrome (DSPS), and seven patients displayed an irregular sleep-wake pattern (ISWP). All patients exhibited a 24-hour period of melatonin rhythm, and those with DSPS exhibited a 24-hour periodicity oral-temperature rhythm. However, three of the seven patients with ISWP lacked such a daily rhythm. Furthermore, the patients with ISWP exhibited smaller amplitudes of temperature rhythm. Given that the authors found that as many as $36 \%$ of their particular sample had been diagnosed with insomnia when in fact they had a CRSD, they concluded that CRSDs may be a relatively frequent sleep disorder among these patients, and that misdiagnosis of patients with CRSDs as insomniacs may lead to inappropriate prescription of hypnotic medications.

Quinto and colleagues ${ }^{52}$ described the case of posttraumatic DSPS in a 48 -year-old male, 4 years following TBI. (Note: the authors use the term cerebral concussion; however, as the patient was in coma for "several days," we will consider this to be a severe TBI [sTBI]). Although the patient was 4 years postinjury, the emergence of his sleep onset insomnia was during the acute phase of recovery. Since his injury, it took him 1 to 2 hours to fall asleep. He reported through a sleep diary that he would go to bed between 3:00 and 5:00 a.m., awaken after 1 to 1-and-a-half hours, return to sleep, and awaken between 11:00 a.m. and 12:00 p.m. These findings were confirmed by actigraphy. The patient did not have any other medical or psychiatric disorders. Circadian rhythms are quite strict and are in fact slightly less than 24 hours in humans. The "circadian clock" located in the suprachiasmatic nucleus (SCN) of the hypothalamus serves to keep physiologic functions in synchrony with each other and with the environmental light/dark cycle, thus making a small daily phase advance to entrain to the 24-hour day. A decreased ability to make this phase advance in response to environmental cues is thought to be the underlying pathophysiology behind DSPS, and the authors of this study postulate that damage to the SCN sustained during trauma may result in circadian cycle disorder.

\section{Pediatrics}

We identified six studies, five of which were of moderate quality, which reported sleep complaints in children and adolescents. Five of these studies focused specifically on mTBI. In a 2001 study of 19 adolescents 3 years following minor head injury (mHI), Kaufman and colleagues ${ }^{53}$ reported that in comparison with healthy controls, headinjured teens had significantly reduced periods of sleep time, total sleep time, and sleep efficiency, as well as an increased number and length of awakenings, objectively measured by PSG and 
TABLE 7

\section{Practice Points}

1. It may be of benefit to measure hypocretin-1 in patients with moderate-severe TBI (who are being monitored for Intracranial Pressure [ICP]) during the acute stage as a potential marker/risk factor for the emergence of sleep and wake disorders.

2. Lesions/damage to the hypothalmus are a risk factor for sleep/wake disorders following TBI.

3. Awareness of DSPS as a possible consequence of TBI is important as its symptoms may be overlooked and attributed to the "typical" postconcussion syndrome complex.

4. Proper diagnosis of CRSDs is important as a misdiagnosis of insomnia may lead to inappropriate prescription of hypnotic medication, which "may help patients fall asleep, but would not be efficacious in normalization of the sleep-wake cycle." 51(p. 1139)

5. Treatments with melatonin or bright light may be more appropriate with CRSDs.

\section{Research Questions}

1. "Future research specifically designed to explore the role of neurophysiologic and psychological factors in the emergence of CRSDs following mTBI may improve our understanding of the nature of this association." 51(p.1139) actigraphy over 5 days. Subjectively, those with $\mathrm{mHI}$ reported significantly more difficulties falling asleep, difficulty waking in the morning, restless sleep and parasomnias, fearful awakenings from sleep, and increased daytime sleepiness than did healthy controls. Further investigations by this group of authors ${ }^{54}$ found that in comparison with healthy controls, nearly one third (27/98) of the subjects aged $8-18$ years, with $0.5-6$ years following $\mathrm{mHI}$, had subjective complaints of sleep disturbance and shorter weekend sleep time than did healthy controls. The authors identified that those with $\mathrm{mHI}$ and complaints of sleep disturbance were also more likely to complain of bruxism and manifested a significantly greater BMI. Furthermore, these subjects came from families with a lower level of parental education. Although no information was available regarding preinjury BMI or any patterns of weight gain postinjury, the authors concluded that risk factors for sleep disturbance in $\mathrm{mHI}$ include heavier body mass and lower levels of parental education.

In 2004, Korinthenberg and colleagues ${ }^{55}$ examined predictive factors of posttraumatic syndrome in children (range, 3-13 years) with $\mathrm{mHI}$.
The authors used data from EEG, in addition to a published neurological examination protocol and a structured validated interview at two time points (T1: within 24 hours of the injury, and T2: 4-6 weeks postinjury) to identify that at followup, 23 of 98 patients continued to present with psychiatric complaints, including sleep disturbance and fatigue. These findings did not correlate with the severity of the injury, or somatic, neurologic, or EEG findings immediately postinjury. In their discussion, the authors stated that the "lack of a correlation with the acute concussional symptoms, acute neurological findings and the acute EEG abnormalities suggests that post traumatic complaints in our patients are not primarily caused by organic structural or functional changes. “55(p.116) They noted that parental and patient anxiety is a frequent problem even after $\mathrm{mHI}$, and it correlates with persistent symptoms.

In contrast, however, Necajauskaite and colleagues ${ }^{56}$ studied 102 matched pairs of children aged 4-16 years, including a case group with mTBI and comparison group with mild bodily injury but no mTBI. They found that although $16.7 \%$ of the parents in the case group reported 
sleep disturbances in their children shortly after the trauma (exact time not defined), they did not find any significant differences in subjective reports of sleep disturbance between the two groups in the month prior to follow-up (1-5 years postinjury; mean, 27 months).

In the most recent study to examine sleep in children with mTBI, Milroy and colleagues ${ }^{57}$ compared 18 children aged $7-12$ years with mTBI with 30 children with orthopaedic injuries (average time postinjury, 24 months). These authors used both subjective and objective measures (parental and self-report sleep questionnaires and actigraphy), and while parents reported greater sleep disturbances in the mTBI group, there were no significant differences between the groups with respect to daytime sleepiness, children's self-report of sleep disturbance, and actigraphy results.

We found only one study that examined sleep in children with moderate-severe TBI. Beebe and colleagues ${ }^{58}$ compared parental reports of sleep behaviors in approximately 100 children (aged 6-12 years), 50 with modTBI and 50 with sTBI, with a comparison group of 80 children with orthopaedic injuries. The children were followed longitudinally over three time points at approximately 6,12 , and 48 months postinjury. The authors reported that for both groups, daytime sleepiness and nocturnal sleep duration were increased following TBI. The modTBI group reported higher baseline sleep problems than the sTBI group and the orthopaedic group; however, this declined over the follow-up period (the specific time course was not specified) to a level consistent with the general population (6-9\%). However, the authors reported that the sTBI group displayed an injury-associated increase in sleep problems, with the prevalence nearly doubling from $16 \%$ at baseline to $31 \%$ postinjury (again, specific timelines were not specified). The authors cautioned that these results should be considered as preliminary given the limitations of the outcome measure; however, they concluded that sleep problems emerge after severe pediatric TBI (and are present in the postacute stage in modTBI).

In conclusion, there is emerging conclusive evidence to support the incidence and prevalence of sleep disturbances in children and adolescents following TBI, across all levels of severity. There

\section{TABLE 8}

\section{Practice Points}

1. Children and adolescents with $\mathrm{mTBI} / \mathrm{mHI}$ need to be systematically and routinely assessed for subjective complaints of sleep disturbances and followed-up over time.

2. Children with moderate-severe TBIs should be followed and assessed for emergence of sleep problems, with provisions of appropriate sleep interventions.

3. Adolescents with $\mathrm{mHI}$, higher $\mathrm{BMI}$, and lower levels of parental education are at increased risk for sleep disturbances. Educating adolescents and parents about healthy eating and regular activity may be of benefit.

4. Providing parents with education and information during the acute stage post $\mathrm{mHI}$ may help to alleviate anxiety and improve outcome.

\section{Research Questions}

1. Sleep (and wake) problems in children and adolescents with both mild and moderate-severe TBIs should be evaluated longitudinally by both objective and subjective measures beginning at the onset of injury, to determine any patterns in the developmental course of sleep distrubance. 
is both subjective and objective evidence documenting the prevalence of sleep disturbance in adolescents with mTBI several years postinjury. There is emerging subjective evidence to suggest that sleep disturbances become more prevalent over time in children with sTBI, yet decline over time postinjury in children with modTBI. There is evidence to suggest that sleep problems in children with mTBI do not correlate with initial neurological findings. Clearly, the research on sleep disturbances in children with TBI is in the early stage and warrants further scientific investigation to more clearly identify the nature and patterns of the disturbance, any functional implications, and appropriate interventions.

\section{Neuropsychological Implications}

Sleep disturbances following TBI have been reported to exacerbate cognitive and behavioral sequelae. We identified four studies of moderate quality: three evaluated neuropsychological (and cognitive-communication) function in relation to sleep disorders following TBI, and one documented both sleep and neuropsychological sequelae following nonimpact brain injury. In 2000, Henry and colleagues ${ }^{59}$ retrospectively investigated the neuropsychological and behavioral functioning of 32 adults (aged 18-66 years) with "non-impact brain injury" (i.e., whiplash) up to 65 months postinjury. The authors used a comprehensive battery of standardized neuropsychological and psychological tests to assess cognition, motor skills, and mood functioning. As the study was retrospective, some subjects had had structural neuroimaging, and three had undergone overnight sleep studies, in addition to neurological examination and interview. Results indicated that patients with whiplash injury demonstrated persistent cognitive, behavioral, and emotional dysfunction years postinjury. Fifty-three percent had problems with sleep (not defined) in addition to difficulties with attention, concentration, executive functions, reduced information processing, word finding, sexuality, anxiety, and depression. Of the three subjects who underwent a nocturnal sleep study, sleep maintenance insomnia was identified with altered sleep stage percentages and altered REM rhythms. Although the authors cite methodological limitations as precluding any conclusions regarding causation, finding sleep disturbance following whiplash is not surprising given the shearing injury and potential involvement of the hypothalamus.

In 2004, Mahmood and colleagues ${ }^{60}$ conducted an investigation of the relationship between sleep disturbance and neurocognitive ability in 87 adults with TBI across all levels of severity who had been admitted to a comprehensive outpatient rehabilitation program. The authors conducted a cross-sectional examination of scores on patients' neuropsychological examinations, the BDI-II and the PSQI. They reported that: "as would be expected, PSQI scores and BDI-II scores were significantly correlated because of the partial overlap of depression symptoms and insomnia." ${ }^{60(\mathrm{p} .382)}$ The authors also reported that measures sensitive to higher-order executive functioning and speed of information processing showed a positive relationship with PSQI scores. Their findings supported a hypothesis of a predictive relationship between performance on neuropsychological tests and reports of sleep disturbance in adults with TBI.

In 2007, Wilde and colleagues ${ }^{61}$ examined $^{2}$ the impact of comorbid OSA (diagnosed after the TBI) on the cognitive functioning of 19 patients with TBI, in comparison with 16 patients with TBI but without OSA. The subjects were otherwise comparable in terms of age, education, injury severity, time postinjury, and GCS score (where available). The patients with TBI and OSA performed significantly worse on verbal and visual delayed recall measures, and had more attention lapses than the patients with TBI without OSA. Interestingly, they did not differ on other measures of visual construction and motor and attention tests. The authors concluded that patients with TBI and OSA had greater impairments in sustained attention and memory than did those patients with TBI without OSA.

In 2010, Wiseman-Hakes and colleagues ${ }^{62}$ assessed daily self- and clinician (rehabilitation worker) reports of changes in cognitive and communication functioning, including sustained auditory attention/vigilance, speed of language processing, verbal memory, and communication, in response to a course of pharmacological intervention phased in over 17 weeks for post- 
traumatic hypersomnia. The self-report measure used in this study is the newly developed Daily Cognitive-Communication and Sleep Profile (DCCASP), which is currently undergoing reliability and validity testing (Wiseman-Hakes, 2005, unpublished measure). This case-study participant was a young man in his late teens 11 months after sTBI when the study began. As treatment progressed, the authors found a clear positive relationship between improved quality of sleep and language processing (defined as the ability to follow and participate in conversation), sustained attention/vigilance (defined as the ability to pay attention and concentrate over time), and memory (defined as the ability to remember things that one has heard, done, or seen). This was seen across the different phases of the medication regime $(P<.01)$. A follow-up conducted at 3 years and 8 months indicated that the gains in sleep, daytime wakefulness, and cognitivecommunication function had been maintained. Although the data were limited to one subject, the findings suggest that appropriate, timely, and effective diagnosis, and management of sleep/ wake disturbances following TBI, may facilitate improved cognitive-communication function. The authors concluded that their results need to be corroborated with a larger sample size and the addition of standardized neuropsychological and cognitive-communication assessment measures to clinically evaluate reported functional changes. ${ }^{62}$

\section{Intervention}

The literature on the treatment of sleep disturbances following TBI is relatively limited at this time. This may be a reflection of the complexity of this disorder from a number of perspectives; that is, the lack of a clear etiology in many patients, mixed diagnoses, lack of consistency regarding the developmental course of the disorder, and interactions with secondary issues such as pain and depression. We identified six papers for review in total: four were of moderate quality and two of high quality. Four of the six articles focused upon pharmaceutical management; of these four, one was for the treatment of fatigue and excessive daytime sleepiness following TBI, one was for the treatment of narcolepsy, another was for the treatment of insomnia following TBI and stroke, and only one addressed treatments for a number of different types of sleep disorders commonly associated with TBI. The remaining two articles focused on behavioral intervention specifically for insomnia.

These latter two moderate-quality articles by Ouellet and Morin ${ }^{63,64}$ assessed the efficacy of a protocol of CBT administered by a registered clinical psychologist over a period of 8 weeks. The first paper reports an individual case study and the second reports a single case series. The authors state that cognitive therapy for insomnia consists of "identifying, challenging and altering a set of dysfunctional beliefs and attitudes about sleep," with the objective of "breaking the cycle of insomnia; dysfunctional thoughts and emotional distress that lead to further sleep disturbances."63(p. 1299) The authors do a thorough job of delineating the treatment protocol, which was adapted to take into account the deficits in memory, attention, and processing that can occur following TBI.

The results of the case study are promising; the participant experienced reduced sleep onset latencies and nocturnal awakenings, and an increase in sleep efficiency. These results were well maintained at follow-up at both 1 and 3 months. The authors built upon these initial results by expanding their assessment measures and number of subjects in a single-case series of 11 individuals (six males, five females) with mild to severe TBI. There are a number of relative strengths of this article, despite the small sample size. Subjects were recruited from a variety of locations using a variety of methods, including major rehabilitation centers across Quebec, Canada, as well as through advertisements to TBI associations and local support groups. Furthermore, all levels of injury severity were represented, indicating that although the sample size is small, their sample is likely fairly representative of the post-TBI population. Their results indicated a decline in total overnight wake time relative to baseline, as well as a reduction in overnight sleep variability in 8 of 11 participants. Treatment effects were seen 1-2 weeks after the beginning of therapy. All participants increased their sleep efficiency, and 5 of 11 achieved a sleep efficiency of greater than $90 \%$ at the 3-month follow-up. At posttreatment, 7 of 11 no longer fulfilled the diagnostic criteria 
for insomnia. Based on their results, the authors suggest that insomnia associated with TBI can be successfully improved in a large proportion of cases with short-term cognitive behavioral intervention.

The remaining four papers for review addressed pharmacological intervention. Jha and colleagues ${ }^{65}$ examined the efficacy of modafinil in treating fatigue and excessive daytime sleepiness in individuals with TBI. This high quality, comprehensive, and well-conceived study consisted of a double-blind placebo cross-over trial in which 53 patients with TBI were randomly assigned to receive up to $400 \mathrm{mg}$ of modafinil, or an equal number of inactive placebo tablets. The participants were a minimum of 1 year postinjury, with a severity level such that they required inpatient rehabilitation (no other information regarding levels of severity was provided). For the modafinil regime, patients took $100 \mathrm{mg}$ (one tablet) once per day at noon for 3 days, and then increased to 100 mg 2 times per day for the next 11 days, followed by the maintenance dose of $200 \mathrm{mg}$ twice daily in the morning and at noon. Following the end of the randomized study, both groups were offered a 4-week open label period in which they could receive modafinil using individually clinically monitored titration and a maintenance dosage. However, results indicated that after adjusting for baseline scores and period effects, there were no statistically significant differences between improvements with modafinil versus placebo on any of the outcome measures (Fatigue Severity Scale, the Modified Fatigue Impact Scale, and the ESS). Thus, despite the scientific rigor of this study, there were no consistent and/or persistent clinical improvements.

The next study by Li Pi Shan and Ashworth ${ }^{66}$ focused specifically on insomnia post-TBI and stroke, and aimed to compare the efficacy of lorazepam and zoplicone as treatment options. This study also followed a double-blind cross-over trial involving 18 patients with TBI or stroke (six patients with TBI, six with a right hemisphere stroke, and six with a left hemisphere stroke; no information about severity or time postinjury). Participants were prescribed $0.5-1.0 \mathrm{mg}$ lorazepam at bedtime as needed for 7 days, followed by $3.75-7.5 \mathrm{mg}$ zoplicone at bedtime also as needed. Participants were able to regulate their own dosage from 0 to 0.5 to 1 tablet per night, to "empower them and to simulate what normally happens on our ward." ${ }^{66(p .422)}$ Results indicated no significant differences in total sleep time or in subjective measures of sleep. Furthermore, the authors evaluated cognition using the Mini-Mental State Exam (which in our view is an extremely weak and insensitive measure for this purpose, particularly for the TBI population), and found no changes with either medication. The authors concluded that zoplicone and lorazepam are equally effective in the treatment of insomnia for both populations. However, because the authors do not report any baseline information on sleep, the reader is not able to determine any actual level of improvement and any clinical application of their findings is thus negated, which is another weakness of this study.

The most recent study by Castriotta and colleagues ${ }^{67}$ aimed to determine whether treatment of specific sleep disorders (which they diagnosed by means of PSG) would result in resolution of those disorders and improvement of symptoms and daytime function. The investigators conducted a thorough, high-quality prospective evaluation of 57 unselected patients with TBI, $>3$ months postinjury using PSG, MSLT, ESS, and neuropsychological testing, including the Psychomotor Vigilance Test (PVT), Profile of Mood States, (POMS), and the Functional Outcome of Sleep Questionnaire (FOSQ), prior to and after treatment for OSA (23\% of 22/57), posttraumatic hypersomnia (PTH) (3\%), narcolepsy (5\%), PLM $(7 \%)$, and objective excessive daytime sleepiness (22\%). In total, 22 of 57 subjects had abnormal polysomnograms (39\%). Treatments included continuous positive airway pressure (CPAP) for OSA, modafinil (200 mg) for narcolepsy and PTH, or pramipexole $(0.375 \mathrm{mg})$ for PLM. In terms of response to treatment, the investigators found that the apneas (and hypopneas, as well as snoring) were eliminated by CPAP; however, there was no significant change in MSLT scores. In addition, they found that PLMs were eliminated with pramipexole. One of three narcolepsy subjects and one of two subjects with PTH had resolutions of hypersomnia with modafinil. However, there were no significant changes in FOSQ, POMS, or PVT results after treatment. The authors concluded that treatment of various sleep disorders after TBI may result in polysomnographic resolution, 
without change in sleepiness or neuropsychological function.

Overall, the results of these four studies on pharmacotherapy leave us with somewhat of a clinical conundrum. The results of Castriotta and colleagues ${ }^{67}$ may provide guidance to those treating posttraumatic apnea (and hypopneas), PLMs, and hypersomnia, which makes intuitive sense from a clinical perspective because these disorders are relatively more "clear cut" in their etiology. However, insomnias can be more complex and multi-faceted in their etiology, potentially including a combination of underlying physiological and psychiatric issues, and thus are often harder to manage pharmacologically without a combination of medications. Furthermore, results of the Shan and Ashworth study ${ }^{66}$ lead us to conclude that standardized dosages may not be effective, and that any medication regime needs to be fine tuned to the individual needs of the patient, an important protocol built into the final 4 weeks of the study by Jha and colleagues. ${ }^{65}$ Thus, an ideal treatment regimen may include a trial of cognitive behavior therapy with the addition of pharmacotherapy as needed, specifically tailored to the individuals' symptoms, body weight, and tolerance. In addition, Castriotta and colleagues ${ }^{67}$ reported resolution of polysomnographic findings without associated improvements in daytime function, which suggests that a follow-up study may be of benefit, with an individual case-series design in which additional treatments and/or medications could be added that are specific to each participant. Based on the current findings to date, it is clear that intervention for sleep and wake disorders following TBI is an area of significant need with respect to future research directions.

\section{DISCUSSION}

This systematic review was conducted to summarize the current literature on the topic of sleep and wake disorders following TBI and to critically appraise the research directions. In summary, our findings concur with previous reviews: sleep and wake disorders are a prevalent and complex sequelae of TBI, occurring at all stages across the continuum of recovery from acute, to postacute, to rehabilitation, in both children and adults, and for a number of patients, continuing for many years postinjury, long into the community. These disorders are complex and multi-factorial, and have an impact on neuropsychological functioning and participation in rehabilitation and quality of life. They may evolve from a neurophysiological disruption in brain function to a secondary disorder associated with depression, anxiety, weight gain, and other factors. As such, intervention is equally complex and much more work needs to be done to fully understand the underlying etiologies and to find appropriate treatments. With regard to methodological quality and scientific rigor of the literature, there is a readily observable trend toward higher-quality, more methodologically sound studies as the literature evolves over time. To date, however, most studies have been limited by small sample sizes, large ranges in time postinjury of participants, loss to, or lack of, follow-up, and sample bias due to timing and methods of recruitment. Thus, it is still unclear exactly which members of the TBI population are affected and how the disorder evolves over time. Furthermore, it is unclear why those with mTBI are more likely to present with insomnia or symptoms of insomnia, and whether this is a true manifestation of the disorder or a reflection of other issues such as self-awareness or the challenges of returning to work, school, and/ or the community. We recognize, however, that the current state of the literature also reflects the inherent complexities of conducting clinical research with this highly diverse, heterogeneous population.

\section{A. Limitations}

Although the current review utilized a systematic approach for the critical appraisal and determination of methodological quality of the literature, we recognize a number of limitations in this methodology. All of the articles included have been peer-reviewed and, as such, there is publication bias. Furthermore, while we have attempted to fully capture all of the recent relevant articles with our search criteria, it is possible that we may have missed articles; thus, this review may not be fully representative of the current literature. 
Given constraints of time, we did not contact any of the study authors directly to clarify any points of confusion; our quality rating system is based upon our understanding of the reporting of the methodology and results, and thus may not be truly representative of the actual study design and quality.

\section{B. What This Review Adds}

Despite its limitations, this review adds to the body of knowledge regarding sleep and wake disorders following TBI. To date, it is the most comprehensive review, with the inclusion of literature specific to the pediatric population, and is also the first to attempt to delineate the prevalence and nature of sleep disorders by timing of recruitment and levels of severity across the continuum of recovery. It is also the first review to systematically appraise the methodological quality of the current literature.

\section{Conclusions and Future Research Directions}

Sleep and wake disorders following TBI have received increasingly considerable attention among the clinical and scientific community. Researchers have attempted to quantify the incidence and prevalence of these disorders, as well as to define and describe the nature of the disorder with both subjective and objective measures. Furthermore, attempts have been made to understand the underlying pathophysiological mechanisms and other contributing factors, as well the functional implications and treatment options. However, additional research is needed to fully understand this complex disorder and to identify appropriate and timely interventions. A large, multi-center study is needed that follows all of those with TBI from the acute stage with regular follow-up into the community, such that results can be reliable, valid, and stratified by nature and severity of injury, age, sex, imaging results, and development of the disorder over time. In addition, we recommend that outcomes for either epidemiological or intervention studies should also capture functional outcomes in addition to sleep measures and cognitive measures to fully assess the impact of the intervention. Finally, we concur with statements by Dr. Richard Castriotta in his recent editorial that "careful study will require much effort and many resources," which can best be brought to fruition "through collaborative efforts across multiple centers," with an aim to "elucidate the causes, foster early diagnosis, and develop optimal treatment for these problems." $68(\mathrm{p}$. 177) These continuing efforts will further inform clinical practice and ultimately contribute to the development of practice guidelines for the systematic evaluation and treatment of sleep and wake disorders following TBI.

\section{ACKNOWLEDGMENTS}

This work was supported by the Canadian Institutes of Health Research (CIHR) through a Fellowship in Clinical Research and by the Toronto Rehabilitation Institute, which receives funding under the Provincial Rehabilitation Research Program from the Ministry of Health and Long-Term Care in Ontario, Canada. The views expressed do not necessarily reflect those of the Ministry. None of the authors have any conflicts of interest.

We gratefully acknowledge the assistance of Ms. Reema Farhat and Ms. Sandra Sokoloff, and Ms. Marcia Winterbottom, librarian at the Toronto Rehabilitation Institute, for her assistance with the literature search.

\section{REFERENCES}

1.World Health Organization. Neurological disorders: Public health challenges. [published 2010; cited 2010 Mar 18]. Available from: http://www.who.int/neurology/ en/.

2.French LM, Parkinson GW. Assessing and treating veterans with traumatic brain injury. J Clin Psychol. 2008;64(8):1004-13.

3.United States Department of Defense, Defense and Veterans Brain Injury Center. TBI numbers. [published 2009, cited 2010 Mar 18]. Available from: http://www.dvbic. org.

4.Ouellet MC, Beaulieu-Bonneau S, Morin CM. Insomnia in patients with traumatic brain injury: Frequency, characteristics, and risk factors. J Head Trauma Rehabil. 2006;21(3):199-212.

5.Seyone C, Kara B. Head injuries and sleep. In: Lader MH, Cardinali DP, Pandi-Perumal SR, editors. Sleep and sleep disorders: a neuropsychopharmacological ap- 
proach. New York (NY): Landes Bioscience/Eurekah. com \& Springer Science + Business Media; 2006.

6.Ouellet MC, Savard J, Morin CM. Insomnia following traumatic brain injury: A review. Neurorehabil Neural Repair. 2004;18(4):187-98.

7.Orff H, Ayalon L, Drummond S. Traumatic brain injury and sleep disturbance: a review of current research. J Head Trauma Rehabil. 2009;24(3):155-65.

8.Zeitzer J, Friedman L, O'Hara R. Insomnia in the context of traumatic brain injury. J Rehabil Res Dev. 2009;46(6):827-36.

9.Langlois JA, Rutland-Brown W, Thomas KE. The incidence of traumatic brain injury among children in the United States: differences by race. J Head Trauma Rehabil. 2005;20(3):229-38.

10.Schneier A, Sheilds B, Hostetler S, Xiang H, Smith G. Incidence of pediatric traumatic brain injury and associated hospital resource utilization in the United States. Pediatrics. 2006;118(2):483-92.

11.Centers for Disease Control and Prevention, Division of Injury Response. Traumatic brain injury in the United States: emergency department visits, hospitalizations and deaths. [published 2006; cited 2010 Mar 18]. Available from: www.cdc.gov/injury.

12.Tobler I. Is sleep fundamentally different between mammalian species? Behav Brain Res. 1995;69(1):35-41.

13.Mullington JM. Endocrine function during sleep and sleep deprivation. In: Stickgold R, Walker MP, editors. The neuroscience of sleep. San Diego (CA): Academic Press; 2009. p. 209-212.

14.Maquet P, Smith C, Stickgold R. Sleep and brain plasticity. New York (NY): Oxford University Press Inc; 2003.

15.Walker M. Sleep-dependent memory processing. In: Stickgold R, Walker M, editors. The neuroscience of sleep. San Diego (CA): Academic Press; 2009.

16.Barkoukis TJ, Avidan AY, editors. Review of sleep medicine. Philadelphia (PA): Butterworth, Heinnemann Elsevier Publishing; 2007.

17.Mirmiran M, Ariango R. Role of REM sleep in brain development and plasticity. In: Maquet P, Smith C, Stickgold R, editors. Sleep and brain plasticity. Oxford, United Kingdom: Oxford University Press; 2003.

18.Holm MB. Our mandate for the new millenium: evidencebased practice. Am J Occup Ther. 2000;54(6):575-85.

19.Boschen K, Gargaro J, Gan C, Gerber G, Brandys C. Family interventions after acquired brain injury and other chronic conditions: a critical appraisal of the quality of the evidence. NeuroRehabilitation. 2007;22(1):19-41.

20.Downs S, Black N. The feasibility of creating a checklist for the assessment of the methodological quality both of randomised and non-randomised studies of health care interventions. J Epidemiol Community Health. 1998;52(6):377-84.

21. Law M. Evidence-based rehabilitation: a guide to practice. Thorofare (NJ): SLACK Inc; 2002.

22.Sherrington $\mathrm{C}$, Herbert RD, Maher CG, Moseley AM. PEDro: A database of randomized trials and systematic reviews in physiotherapy. Man Ther. 2000;5(4):223-6.
23.Comper P, Hutchison M, Magrys S, Mainwaring L, Richards D. Evaluating the methodological quality of sports concussion research: a systematic review. Brain Inj. 2010;24(11):1257-71.

24.Baumann CR, Werth E, Stocker R, Ludwig S, Bassetti CL. Sleep-wake disturbances 6 months after traumatic brain injury: a prospective study. Brain. 2007;130(7):187383.

25.Castriotta RJ, Wilde MC, Lai JM, Atanasov S, Masel BE, Kuna ST. Prevalence and consequences of sleep disorders in traumatic brain injury. J Clin Sleep Med. 2007;3(4):349-56.

26.Parcell DL, Ponsford JL, Redman JR, Rajaratnam SM. Poor sleep quality and changes in objectively recorded sleep after traumatic brain injury: a preliminary study. Arch Phys Med Rehabil. 2008;89(5):843-50.

27.Gosselin N, Lassonde M, Petit D, Leclerc S, Mongrain V, Collie A, Montplaisir J. Sleep following sport-related concussions. Sleep Med. 2009;10(1):35-46.

28. Makley M, English J, Drubach D, Kreuz A, Celnik P, Tarwater P. Prevalence of sleep disturbance in closed head injury patients in a rehabilitation unit. Neurorehabil Neural Repair. 2008;22(4):341-7.

29.Parcell DL, Ponsford JL, Rajaratnam SM, Redman JR. Self-reported changes to nighttime sleep after traumatic brain injury. Arch Phys Med Rehabil. 2006;87(2):278-85.

30.Rao V, Spiro J, Vaishnavi S, Rastogi P, Mielke M, Noll K, Cornwell E, Schretlen D, Makley M. Prevalence and types of sleep disturbances acutely after traumatic brain injury. Brain Inj. 2008;22(5):381-6.

31. Verma A, Anand V, Verma NP. Sleep disorders in chronic traumatic brain injury. J Clin Sleep Med. 2007;3(4):357-62.

32.Fichtenberg NL, Zafonte RD, Putnam S, Mann NR, Millard AE. Insomnia in a post-acute brain injury sample. Brain Inj. 2002;16(3):197-206.

33.Castriotta RJ, Lai JM. Sleep disorders associated with traumatic brain injury. Arch Phys Med Rehabil. 2001;82(10):1403-6.

34.Burke DT, Shah MK, Schneider JC, Ahangar B, Al-Aladai $\mathrm{S}$. Sleep-wake patterns in brain injury patients in an acute inpatient rehabilitation hospital setting. J Appl Res. 2004;4(2):239-44.

35.Beetar JT, Guilmette TJ, Sparadeo FR. Sleep and pain complaints in symptomatic traumatic brain injury and neurologic populations. Arch Phys Med Rehabil. 1996;77(12):1298-302.

36.Schreiber S, Barkai G, Gur-Hartman T, Peles E, Tov N, Dolberg OT, Pick CG. Long-lasting sleep patterns of adult patients with minor traumatic brain injury (mTBI) and non-mTBI subjects. Sleep Med. 2008;9(5):481-7.

37.Williams BR, Lazic SE, Ogilvie RD. Polysomnographic and quantitative EEG analysis of subjects with long-term insomnia complaints associated with mild traumatic brain injury. Clin Neurophysiol. 2008;119(2):429-38.

38.Chaput G, Giguere J, Chauny J, Denis R, Lavigne G. Relationship among subjective sleep complaints, head- 
aches, and mood alterations following mild traumatic brain injury. Sleep Med. 2009;10(7):713-6.

39. Watson NF, Dikmen S, Machamer J, Doherty M, Temkin N. Hypersomnia following traumatic brain injury. J Clin Sleep Med. 2007;3(4):363-8.

40.Clinchot DM, Bogner J, Mysiw WJ, Fugate L, Corrigan J. Defining sleep disturbance after brain injury. Am J Phys Med Rehabil. 1998;77(4):291-5.

41.Fichtenberg NL, Millis SR, Mann NR, Zafonte RD, Millard AE. Factors associated with insomnia among post-acute traumatic brain injury survivors. Brain Inj. 2000;14(7):659-67.

42.Masel BE, Scheibel RS, Kimbark T, Kuna ST. Excessive daytime sleepiness in adults with brain injuries. Arch Phys Med Rehabil. 2001;82(11):1526-32.

43. Worthington AD, Melia Y. Rehabilitation is compromised by arousal and sleep disorders: Results of a survey of rehabilitation centres. Brain Inj. 2006;20(3):327-32.

44.Ouellet MC, Morin CM. Subjective and objective measures of insomnia in the context of traumatic brain injury: a preliminary study. Sleep Med. 2006;7(6):486-97.

45.Dvorak J, McCrory P, Kirkendall DT. Head injuries in the female football player: incidence, mechanisms, risk factors and management. Br J Sports Med. 2007;41(Suppl 1):i44-6.

46.Covassin T, Swanik CB, Sachs ML. Sex differences and the incidence of concussions among collegiate athletes. J Athl Train. 2003;38(3):238-44.

47.Covassin T, Swanik CB, Sachs M, Kendrick Z, Schatz P, Zillmer E, Kaminaris C. Sex differences in baseline neuropsychological function and concussion symptoms of collegiate athletes. Br J Sports Med. 2006;40(11):923-7.

48.Marshall LF, Marshall SB, Klauber MR, Van Berkum Clark M, Eisenberg H, Jane JA, Luerssen TG, Marmarou A, Foulkes MA. The diagnosis of head injury requires a classification based on computed axial tomography. J Neurotrauma. 1992;9(Supp1 1):S287-92.

49.Hays RD, Martin SA, Sesti AM, Spritzer KL. Psychometric properties of the Medical Outcomes Study Sleep measure. Sleep Med. 2005;6(1):41-4.

50.Baumann CR, Stocker R, Imhof HG, Trentz O, Hersberger M, Mignot E, Bassetti CL. Hypocretin-1 (orexin A) deficiency in acute traumatic brain injury. Neurology. 2005;65(1):147-9.

51.Ayalon L, Borodkin K, Dishon L, Kanety H, Dagan Y. Circadian rhythm sleep disorders following mild traumatic brain injury. Neurology. 2007;68(14):1136-40.

52.Quinto C, Gellido C, Chokroverty S, Masdeu J. Posttraumatic delayed sleep phase syndrome. Neurology. 2000;54(1):250-2.

53.Kaufman Y, Tzischinsky O, Epstein R, Etzioni A, Lavie P, Pillar G. Long-term sleep disturbances in adolescents after minor head injury. Pediatr Neurol. 2001;24(2):129-34.

54.Pillar G, Averbooch E, Katz N, Peled N, Kaufman Y, Shahar E. Prevalence and risk of sleep disturbances in adolescents after minor head injury. Pediatr Neurol. 2003;29(2):131-5.

55.Korinthenberg R, Schreck J, Weser J, Lehmkuhl G. Posttraumatic syndrome after minor head injury cannot be predicted by neurological investigations. Brain Dev. 2004;26(2):113-7.

56. Necajauskaite O, Endziniene M, Jureniene $\mathrm{K}$. The prevalence, course and clinical features of postconcussion syndrome in children. Medicina (Kaunas). 2005;41(6):457-64.

57.Milroy G, Dorris L, McMillan TM. Sleep disturbances following mild traumatic brain injury in childhood. J Pediatr Psychol. 2008;33(3):242-7.

58.Beebe DW, Krivitzky L, Wells CT, Wade SL, Taylor HG, Yeates KO. Brief report: parental report of sleep behaviors following moderate or severe pediatric traumatic brain injury. J Pediatr Psychol. 2007;32(7):845-50.

59.Henry GK, Gross HS, Herndon CA, Furst CJ. Nonimpact brain injury: neuropsychological and behavioral correlates with consideration of physiological findings. Appl Neuropsychol. 2000;7(2):65-75.

60.Mahmood O, Rapport LJ, Hanks RA, Fichtenberg NL. Neuropsychological performance and sleep disturbance following traumatic brain injury. J Head Trauma Rehabil. 2004;19(5):378-90.

61. Wilde MC, Castriotta RJ, Lai JM, Atanasov S, Masel BE, Kuna ST. Cognitive impairment in patients with traumatic brain injury and obstructive sleep apnea. Arch Phys Med Rehabil. 2007;88(10):1284-8.

62.Wiseman-Hakes C, Murray BJ, Victor C. Sleep/wake disturbance following traumatic brain injury: Impact on functional recovery of cognition and communication: a case study. Brain Inj. 2010;24 (3):376.

63. Ouellet MC, Morin CM. Cognitive behavioral therapy for insomnia associated with traumatic brain injury: a single-case study. Arch Phys Med Rehabil. 2004;85(8):1298-302.

64. Ouellet MC, Morin CM. Efficacy of cognitive-behavioral therapy for insomnia associated with traumatic brain injury: a single-case experimental design. Arch Phys Med Rehabil. 2007;88(12):1581-92.

65.Jha A, Weintraub A, Allshouse A, Morey C, Cusick C, Kittelson J, Harrison-Felix C, Whiteneck G, Gerber D. A randomized trial of modafinil for the treatment of fatigue and excessive daytime sleepiness in individuals with chronic traumatic brain injury. J Head Trauma Rehabil. 2008;23(1):52-63.

66.Li Pi Shan RS, Ashworth NL. Comparison of lorazepam and zopiclone for insomnia in patients with stroke and brain injury: A randomized, crossover, double-blinded trial. Am J Phys Med Rehabil. 2004;83(6):421-7.

67.Castriotta RJ, Atanasov S, Wilde MC, Masel BE, Lai JM, Kuna ST. Treatment of sleep disorders after traumatic brain injury. J Clin Sleep Med. 2009;5(2):137-44.

68. Castriotta RJ. Collaboration in research involving traumatic brain injury and sleep disorders. J Clin Sleep Med. 2008;4(2):177. 